Physics

Physics Research Publications

\title{
Multiwaveband polarimetric observations of 15 active galactic nuclei at high frequencies: Correlated polarization behavior
}

S. G. Jorstad, A. P. Marscher, J. A. Stevens, P. S. Smith, J. R. Forster, W. K. Gear, T. V. Cawthorne, M. L. Lister, A. M. Stirling, J. L. Gomez, J. S. Greaves, and E. I. Robson 


\title{
MULTIWAVEBAND POLARIMETRIC OBSERVATIONS OF 15 ACTIVE GALACTIC NUCLEI AT HIGH FREQUENCIES: CORRELATED POLARIZATION BEHAVIOR
}

\author{
Svetlana G. Jorstad, ${ }^{1,2}$ Alan P. Marscher, ${ }^{1} \mathrm{~J}_{\text {ason A. Stevens, }}{ }^{3}$ Paul S. Smith, ${ }^{4}$ James R. Forster, ${ }^{5}$ \\ Walter K. Gear, ${ }^{6}$ Timothy V. Cawthorne, ${ }^{7}$ Matthew L. Lister, ${ }^{8}$ Alastair M. Stirling, ${ }^{9}$ \\ José L. Gómez, ${ }^{10} \mathrm{JanE}_{\text {S. Greaves, }}{ }^{11}$ and E. IAN Robson ${ }^{12}$ \\ Received 2007 March 7; accepted 2007 May 16
}

\begin{abstract}
We report on multifrequency linear polarization monitoring of 15 active galactic nuclei containing highly relativistic jets with apparent speeds from $\sim 4 c$ to $>40 c$. The measurements were obtained at optical, $1 \mathrm{~mm}$, and $3 \mathrm{~mm}$ wavelengths, and at $7 \mathrm{~mm}$ with the Very Long Baseline Array. The data show a wide range in degree of linear polarization among the sources, from $<1 \%$ to $>30 \%$, and interday polarization variability in individual sources. The polarization properties suggest separation of the sample into three groups with low, intermediate, and high variability of polarization in the core at $7 \mathrm{~mm}$ (LVP, IVP, and HVP, respectively). The groups are partially associated with the common classification of active galactic nuclei as radio galaxies and quasars with low optical polarization (LVP), BL Lacertae objects (IVP), and highly optically polarized quasars (HVP). Our study investigates correlations between total flux, fractional polarization, and polarization position angle at the different wavelengths. We interpret the polarization properties of the sources in the sample through models in which weak shocks compress turbulent plasma in the jet. The differences in the orientation of sources with respect to the observer, jet kinematics, and abundance of thermal matter external to the jet near the core can account for the diversity in the polarization properties. The results provide strong evidence that the optical polarized emission originates in shocks, most likely situated between the 3 and $7 \mathrm{~mm}$ VLBI cores. They also support the idea that the $1 \mathrm{~mm}$ core lies at the edge of the transition zone between electromagnetically dominated and turbulent hydrodynamic sections of the jet.
\end{abstract}

Key words: BL Lacertae objects: individual $(1803+784,1823+568,3 \mathrm{C}$ 66A, BL Lac, OJ 287) galaxies: active - galaxies: individual (3C 111, 3C 120) - galaxies: jets - polarization quasars: individual $(0420-014,0528+134,3 \mathrm{C} 273$, 3C 279, 3C 345, 3C 454.3, CTA 102, PKS 1510-089)

Online material: machine-readable tables

\section{INTRODUCTION}

Magnetic fields play a prominent role in the physical processes that occur in the jets of active galactic nuclei (AGNs). The leading model for jet production, acceleration, and collimation involves poloidal magnetic fields that are wound up by the differential rotation of a rotating disk or ergosphere surrounding a central supermassive black hole (e.g., McKinney 2006; Meier et al. 2001).

\footnotetext{
${ }^{1}$ Institute for Astrophysical Research, Boston University, 725 Commonwealth Avenue, Boston, MA 02215-1401,USA; jorstad@bu.edu, marscher@bu.edu.

2 Sobolev Astronomical Institute, St. Petersburg State University, Universitetskij Prospekt 28, 198504 St. Petersburg, Russia.

${ }^{3}$ Centre for Astrophysics Research, Science and Technology Centre, University of Hertfordshire, College Lane, Herts AL10 9AB, UK; j.a.stevens@herts.ac.uk.

4 Steward Observatory, The University of Arizona, Tucson, AZ 85721, USA; psmith@as.arizona.edu.

${ }^{5}$ Hat Creek Observatory, University of California, Berkeley, 42231 Bidwell Road, Hatcreek, CA 96040, USA; rforster@astro.berkeley.edu.

${ }^{6}$ School of Physics and Astronomy, Cardiff University, 5, The Parade, Cardiff CF2 3YB, Wales, UK; walter.gear@astro.cf.ac.uk.

7 Centre for Astrophysics, University of Central Lancashire, Preston PR1 2HE, UK; tvcawthorne@uclan.ac.uk.

8 Department of Physics, Purdue University, 525 Northwestern Avenue, West Lafayette, IN 47907-2036, USA; mlister@physics.purdue.edu.

9 Jodrell Bank Observatory, University of Manchester, Macclesfield, Cheshire SK119DL, UK; ams@jb.man.ac.uk.

${ }^{10}$ Insituto de Astrofísica de Andalucía (CSIC), Apartado 3004, Granada 18080, Spain;jlgomez@iaa.es.

${ }_{11}$ School of Physics and Astronomy, University of St. Andrews, North Haugh, St. Andrews, Fife KY16 9SS, UK; jsg5@st-and.ac.uk.

12 Astronomy Technology Centre, Royal Observatory, Blackford Hill, Edinburgh EH9 3HJ, UK; eir@roe.ac.uk.
}

The twisted field propagates outward as Poynting flux in the polar directions, with eventual conversion into a well-focused relativistic plasma flow (e.g., Vlahakis \& Königl 2004; Meier \& Nakamura 2006). Within this zone, the magnetic field should maintain a tight helical pattern.

Beyond the jet acceleration region - which may extend over hundreds or thousands of gravitational radii from the black hole (Vlahakis \& Königl 2004; Marscher 2006) — the jet may become turbulent or subject to velocity shear. In the former case, the magnetic field should be chaotic, with any line of sight passing through many turbulent cells and significant differences in both strength and direction of the field in adjacent cells. In contrast, velocity shear stretches and orders the field lines along the flow (e.g., Laing 1980). Shock waves passing through the flow (or vice versa) will compress the component of the field that is parallel to the shock front, which imposes order even on a magnetic field that is completely chaotic in front of the shock.

The geometry and degree of order of the magnetic field are therefore key indicators of the physical conditions in a jet. Because the primary emission mechanism at radio to optical wavelengths is synchrotron radiation, the linear polarization of the continuum can be used as a probe of the magnetic field. We can also use the fractional polarization and direction of the electric vector position angle (EVPA) to identify distinct features in the jet that are observed at different wavelengths. The most prominent features on VLBI images of jets in radio-loud AGNs are (1) the core, which is the bright, very compact section at the narrow end of a one-sided jet, and (2) condensations in the flow that appear as bright knots, often called "components" of the jet. The core 
likely lies some distance from the central engine of the AGN, probably either near the end or beyond the zone of acceleration and collimation of the jet (Marscher 2006). The knots usually separate from the core at apparent superluminal speeds, but roughly stationary knots are also present in many jets, perhaps representing standing shocks (e.g., Jorstad et al. 2001, 2005, hereafter J05; Kellermann et al. 2004; Lister 2006). The apparent speeds of the components can exceed $\beta_{\text {app }} \sim 40 c(\mathrm{~J} 05)$, which requires that the Lorentz factor of the flow exceeds $\beta_{\text {app }}$. The leading model identifies the moving knots as propagating shocks, either transverse to the jet axis (e.g., Hughes et al. 1985, 1989; Marscher \& Gear 1985) or at an oblique angle (Hughes 2005). Polarization studies can help to determine whether this model is viable.

The standard paradigm of transverse shocks propagating down a relativistic jet can explain many aspects of the time variability of the brightness, polarization, and structure at radio wavelengths (e.g., Hughes et al. 1985, 1989; Cawthorne \& Wardle 1988; Wardle et al. 1994). However, this model makes predictions - e.g., that the magnetic field of a knot should be transverse to the jet axis - that often do not match observations. Another possibility is that there is a systematically ordered component of the magnetic field, for example one with a helical geometry (e.g., Lyutikov et al. 2005), in the jet that modulates its brightness and polarization variability. In fact, a helical magnetic field is required in the magnetohydrodynamic jet launching models (Meier et al. 2001; Vlahakis \& Königl 2004). Gabuzda (2006) provided some support for such a geometry in recent low-frequency VLBI observations of Faraday rotation in AGN jets. However, this structure can be related to the magnetic field in the Faraday screen surrounding the jet since high-frequency radio polarization mapping reveals aspects that are difficult to explain by a helical field (Hughes 2005; Zavala \& Taylor 2005).

The situation is even more confusing in the optical and near-IR regions, where variations in flux and polarization are often extremely rapid, and submilliarcsecond resolution is not available (e.g., Hagen-Thorn 1980; Moore et al. 1987; Smith et al. 1987; Mead et al. 1990). Furthermore, existing optical data indicate that the connection between variations in brightness and polarization is tenuous (e.g., Smith 1996). These factors complicate interpretation of the optical/near-IR emission, since detailed models are needed but the data are not extensive enough to guide them. An alternative approach is to investigate the global polarization behavior across a sizable range of the electromagnetic spectrum. Such studies (Wills et al. 1992; Gabuzda et al. 1996, 2006; Lister $\&$ Smith 2000) have produced convincing evidence for a connection between the radio and optical polarization properties of AGNs, suggestive of a common, probably cospatial, origin for the emission at these two wave bands. However, such studies have been based on single-epoch measurements, whereas the investigation we present here involves multiepoch observations of 15 objects. This allows us to use both the time and frequency domains to explore the geometry and degree of ordering of the magnetic field and other properties of the emission regions in relativistic jets.

Bright jets with high apparent superluminal motion are a prevalent feature of blazars, a classification that includes BL Lac objects and optically violently variable quasars (OVVs) that are quite rare in the general AGN population (Kellermann et al. 2004; Lister 2006). The prominence of the jets from radio to optical wavelengths and the pronounced variability of their polarized emission makes blazars and radio galaxies with blazar-like behavior ideal objects for probing the magnetic fields in jets through multiepoch polarization studies.
We have carried out a 3 yr monitoring program of 15 radioloud, highly variable AGNs. The program combines roughly bimonthly, high-resolution polarized and total-intensity radio images with optical, submillimeter-wave, and millimeter-wave polarization observations performed at many of the same epochs. The sample includes objects that are usually brighter than about $2 \mathrm{Jy}$ at $7 \mathrm{~mm}$ and $1 \mathrm{Jy}$ at $1 \mathrm{~mm}$, with a mixture of quasars $(0420-$ 014, 0528+134, 3C 273, 3C 279, 1510-089, 3C 345, CTA 102, and 3C 454.3), BL Lac objects (3C 66A, OJ 287, 1803+784, $1823+568$, and BL Lac), and radio galaxies with bright compact radio jets (3C 111 and 3C 120). In J05, we investigated the properties of the jets on milliarcsecond scales and determined the apparent velocities of more than 100 jet features. For the majority of these components, we derived Doppler factors using a new method based on comparison of the timescale of decline in flux density with the light travel time across the emitting region. This allowed us to estimate the Lorentz factors, as well as viewing and opening angles for the jets of all of the sources in our sample. Here we apply these parameters in our interpetation of the multifrequency polarization properties of the sources. Because of difficulties in coordinating the schedules at the different telescopes, our observations at different wavelengths are contemporaneous (within 2 days to 2 weeks of each other) rather than exactly simultaneous. Nevertheless, the program provides the richest multiepoch, multi-wave band polarization data set compiled to date.

\section{OBSERVATIONS AND DATA REDUCTION}

We have measured the total flux densities and polarization of the objects in our sample in four spectral regions: at $7 \mathrm{~mm}$ (43 GHz), $3 \mathrm{~mm}$ (86 GHz), 0.85/1.3 $\mathrm{mm}(350 / 230 \mathrm{GHz})$, and optical wavelengths (an effective wavelength of $\sim 600-700 \mathrm{~nm}$ ).

\subsection{Optical Polarization Observations}

We carried out optical polarization and photometric measurements at several epochs using the Two-Holer Polarimeter/ Photometer (Sitko et al. 1985) with the Steward Observatory $1.5 \mathrm{~m}$ telescope located on Mount Lemmon, Arizona, and the $1.55 \mathrm{~m}$ telescope on Mount Bigelow, Arizona. This instrument uses a semiachromatic half-wave plate spinning at $20.65 \mathrm{~Hz}$ to modulate incident polarization and a Wollaston prism to direct orthogonally polarized beams to two RCA C31034 GaAs photomultiplier tubes. For all polarization measurements we used either a $4.3^{\prime \prime}$ or $8.0^{\prime \prime}$ circular aperture and, except for 3 C 273 , no filter. The unfiltered observations sample the polarization between $\sim 320$ and $890 \mathrm{~nm}$, with an effective central wavelength of $\sim 600$ $700 \mathrm{~nm}$, depending on the spectral shape of the observed object. In the case of 3 C 273 a Kron-Cousins $R$ filter $\left(\lambda_{\text {eff }} \sim 640 \mathrm{~nm}\right)$ was employed to avoid major unpolarized emission-line features (Smith et al. 1993). We accomplished sky subtraction by nodding the telescope to a nearby $\left(<30^{\prime \prime}\right)$, blank patch of sky several times during each observation. Data reduction of the polarimetry followed the procedure described in Smith et al. (1992).

When conditions were photometric, we obtained differential $V$-band photometry ( $R$-band photometry in the case of 3C 273) of several objects, employing either an $8.0^{\prime \prime}$ or $16.0^{\prime \prime}$ circular aperture. We used comparison stars in the fields of the AGNs (Smith et al. 1985; Smith \& Balonek 1998) to calibrate nearly all of the photometry. We determined the $V$ magnitudes of $3 \mathrm{C} 111$ and 3C 120 on 1999 February 13 from the photometric solution provided by observations of equatorial standard stars (Landolt 1983).

Table 1 lists the results of the optical observations. The columns correspond to (1) source, (2) epoch of observation, (3) filter bandpass of the photometry, (4) apparent magnitude, (5) flux density, 
TABLE 1

Optical Polarization Data

\begin{tabular}{|c|c|c|c|c|c|c|c|}
\hline $\begin{array}{l}\text { Source } \\
\text { (1) }\end{array}$ & $\begin{array}{l}\text { Epoch } \\
\text { (2) }\end{array}$ & $\begin{array}{l}\text { Photometry Filter } \\
\text { (3) }\end{array}$ & $\begin{array}{l}\text { Mag } \\
\text { (4) }\end{array}$ & $\begin{array}{c}I_{\mathrm{opt}} \\
(\mathrm{mJy}) \\
(5)\end{array}$ & $\begin{array}{l}\text { Polarimetry Filter } \\
\text { (6) }\end{array}$ & $\begin{array}{c}m_{\mathrm{opt}} \\
(\%) \\
(7)\end{array}$ & $\begin{array}{c}\chi_{\mathrm{opt}} \\
(\mathrm{deg}) \\
(8)\end{array}$ \\
\hline \multirow[t]{9}{*}{ 3C 66A....................... } & 1999 Feb 12 & $\ldots$ & $\ldots$ & $\ldots$ & $W$ & $13.8 \pm 0.2$ & $37.2 \pm 0.3$ \\
\hline & 1999 Feb 13 & $V$ & $14.70 \pm 0.02$ & $4.81 \pm 0.08$ & $W$ & $13.3 \pm 0.2$ & $45.2 \pm 0.4$ \\
\hline & 1999 Feb 14 & $V$ & $14.74 \pm 0.02$ & $4.63 \pm 0.08$ & $W$ & $14.5 \pm 0.2$ & $47.4 \pm 0.3$ \\
\hline & 2000 Sep 27 & $V$ & $14.78 \pm 0.02$ & $4.46 \pm 0.08$ & $W$ & $29.7 \pm 0.2$ & $17.2 \pm 0.2$ \\
\hline & 2000 Sep 28 & $V$ & $14.81 \pm 0.02$ & $4.35 \pm 0.09$ & $W$ & $26.8 \pm 0.3$ & $17.1 \pm 0.4$ \\
\hline & 2000 Sep 29 & $\ldots$ & $\ldots$ & $\ldots$ & $W$ & $24.2 \pm 0.3$ & $13.4 \pm 0.4$ \\
\hline & 2000 Nov 30 & $V$ & $14.40 \pm 0.02$ & $6.31 \pm 0.11$ & $W$ & $23.7 \pm 0.1$ & $25.4 \pm 0.1$ \\
\hline & 2000 Dec 1 & $\ldots$ & $\ldots$ & $\ldots$ & $W$ & $22.8 \pm 0.2$ & $25.1 \pm 0.2$ \\
\hline & 2001 Jan 19 & $V$ & $14.43 \pm 0.02$ & $6.16 \pm 0.10$ & $W$ & $24.6 \pm 0.1$ & $10.1 \pm 0.1$ \\
\hline \multirow[t]{6}{*}{ 3C $111 \ldots \ldots \ldots \ldots$} & 1999 Feb 13 & $V$ & $18.95 \pm 0.12$ & $0.10 \pm 0.02$ & $W$ & $3.1 \pm 0.8$ & $126 \pm 4$ \\
\hline & 2000 Apr 3 & $\ldots$ & $\ldots$ & $\ldots$ & $W$ & $0.8 \pm 0.8$ & $106 \pm 5$ \\
\hline & 2000 Sep 27 & $\ldots$ & $\ldots$ & $\ldots$ & $W$ & $1.5 \pm 0.6$ & $104 \pm 3$ \\
\hline & 2000 Nov 30 & $\ldots$ & $\ldots$ & $\ldots$ & $W$ & $1.9 \pm 0.5$ & $131 \pm 3$ \\
\hline & 2000 Dec 1 & $\ldots$ & $\ldots$ & $\ldots$ & $W$ & $1.6 \pm 0.8$ & $110 \pm 5$ \\
\hline & 2001 Jan 19 & $\ldots$ & $\ldots$ & $\ldots$ & $W$ & $1.3 \pm 0.4$ & $89 \pm 3$ \\
\hline
\end{tabular}

NoтE.-Table 1 is published in its entirety in the electronic edition of the Astronomical Journal. A portion is shown here for guidance regarding its form and content.

$I_{\text {opt }}$, (6) filter bandpass of the polarimetry, where $W$ denotes unfiltered, or "white-light" measurements, (7) degree of linear polarization, $m_{\mathrm{opt}}$, and (8) polarization position angle $\chi_{\mathrm{opt}}$. We have corrected the degree of linear polarization for the statistical bias inherent in this positive-definite quantity by using the method of Wardle \& Kronberg (1974). Usually this correction is negligible because of the high signal-to-noise ratio $(\mathrm{S} / \mathrm{N})$ of most measurements.

Because of the high observed optical polarization levels and high Galactic latitudes of the majority of the sources, interstellar polarization (ISP) is not a significant concern in most cases. Interstellar polarization from dust within the Milky Way galaxy does appear to be a major component of the observed optical polarization for $3 \mathrm{C} 111\left(b=-8.8^{\circ}\right)$ and $3 \mathrm{C} 120\left(b=-27.4^{\circ}\right)$. A star $\sim 50^{\prime \prime}$ west of $3 \mathrm{C} 111$ shows very high polarization $(m=3.6 \% \pm$ $0.2 \% ; \chi=123^{\circ} \pm 2^{\circ}$ ); this was used to correct the polarimetry for ISP in the line of sight to the radio galaxy. The corrected measurements suggest that the intrinsic polarization of $3 \mathrm{C} 111$ was typically $<3 \%$ throughout the monitoring campaign. The average polarization for five stars within $8^{\prime}$ of 3C 120 yields $m=$ $1.22 \% \pm 0.06 \%$ and $\chi=98^{\circ} \pm 1^{\circ}$. We have used this ISP estimate to correct the observed polarization of 3C 120 for interstellar polarization and, as for $3 \mathrm{C} \mathrm{111,} \mathrm{list} \mathrm{the} \mathrm{corrected} \mathrm{measurements}$ in Table 1 . The corrected values indicate that $3 \mathrm{C} 120$ had very low polarization $\left(m_{\mathrm{opt}}<0.5 \%\right)$ throughout the monitoring program.

Impey et al. (1989) have determined the interstellar polarization along the line of sight to 3C 273 to be small, but the low levels of polarization observed for this quasar require that this estimate of the ISP be subtracted from the $R$-band measurements. Table 1 lists the corrected polarization for 3C 273.

\subsection{Submillimeter Polarization Observations}

We performed observations at 1.35 and $0.85 \mathrm{~mm}$ with the James Clerk Maxwell Telescope (JCMT) located on Mauna Kea, Hawaii using the Submillimeter Common User Bolometer Array (SCUBA; Holland et al. 1999) and its polarimeter (Greaves et al. 2003). The initial plan was to observe exclusively at $1.35 \mathrm{~mm}$ because the sources are almost always brighter, the atmospheric opacity is lower, and the sky is more stable than at $0.85 \mathrm{~mm}$. However, failure of the SCUBA filter drum in 1999 November forced a switch to $0.85 \mathrm{~mm}$ thereafter. The polarization properties of blazars tend to be very similar at millimeter and submillimeter wavelengths (Nartallo et al. 1998), so the modest change in wavelength should not affect our analysis. For convenience we refer to the data obtained with the JCMT as $1 \mathrm{~mm}$ data.

The SCUBA polarimeter consists of a rotating quartz halfwave plate and a fixed analyzer. During an observation, the waveplate is stepped through 16 positions, and photometric data are taken at each position. One rotation takes $\sim 360$ s to complete, and the procedure results in a sinusoidally modulated signal from which the Stokes parameters are extracted. A typical observation consists of 5-10 complete rotations of the waveplate. We achieved flux calibration in the standard manner with observations of planets or JCMT secondary calibrators. We measured the instrumental polarization $(\sim 1 \%)$ during each run by making observations of a compact planet, usually Uranus, which is assumed to be unpolarized at millimeter/submillimeter wavelengths.

We performed the initial (nod compensation, flat-field, extinction correction, and sky noise removal, if appropriate) and polarimetric stages of the data reduction using the standard SCUBA data reduction packages SURF and SIT. ${ }^{13}$ The Stokes parameters were extracted by fitting sinusoids to the data, either half cycle (8 points), resulting in two estimates, or full cycle (16 points), yielding only one estimate but generally giving better results with noisy data. We removed spurious measurements by performing a Kolmogorov-Smirnov test on the collated data and then calculated the degree and position angle of the polarization, corrected for instrumental polarization and parallactic angle. Table 2 lists the data, with the columns corresponding to (1) source, (2) epoch of observation, (3) wavelength $\lambda$, (4) flux density $I_{1 \mathrm{~mm}},(5)$ degree of linear polarization $m_{1 \mathrm{~mm}}$, and (6) polarization position angle $\chi_{1 \mathrm{~mm}}$. As with the optical measurements, the values of $m_{1 \mathrm{~mm}}$ have been corrected for statistical bias.

\section{3. $3 \mathrm{~mm}$ Polarization Observations}

From 2000 April to 2001 April we monitored the sources at $86 \mathrm{GHz}$ using the linear polarization system on the the BerkeleyIllinois-Maryland Array (BIMA) in Hat Creek, California during

\footnotetext{
${ }^{13}$ See http://www.jach.hawaii.edu/JCMT/continuum/data/reduce.html.
} 
TABLE 2

JCMT Polarization Data

\begin{tabular}{|c|c|c|c|c|c|}
\hline $\begin{array}{c}\text { Source } \\
\text { (1) }\end{array}$ & $\begin{array}{c}\text { Epoch } \\
\text { (2) }\end{array}$ & $\begin{array}{c}\lambda \\
(\mathrm{mm}) \\
(3)\end{array}$ & $\begin{array}{c}I_{1 \mathrm{~mm}} \\
(\mathrm{Jy}) \\
(4)\end{array}$ & $\begin{array}{c}m_{1 \mathrm{~mm}} \\
(\%) \\
(5)\end{array}$ & $\begin{array}{c}\chi_{1 \mathrm{~mm}} \\
(\mathrm{deg}) \\
(6)\end{array}$ \\
\hline \multirow[t]{11}{*}{ 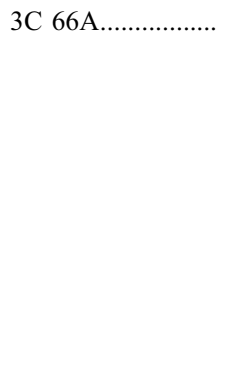 } & 1998 May 15 & 1.35 & $0.38 \pm 0.04$ & $36 \pm 4$ & $24 \pm 2$ \\
\hline & 1998 Jul 17 & 1.35 & $0.41 \pm 0.04$ & $19 \pm 4$ & $176 \pm 6$ \\
\hline & 1998 Sep 30 & 1.35 & $0.45 \pm 0.04$ & $14 \pm 2$ & $56 \pm 4$ \\
\hline & 1998 Nov 23 & 1.35 & $0.41 \pm 0.04$ & $(11 \pm 5)$ & $(172 \pm 10)$ \\
\hline & 1998 Dec 13 & 1.35 & $0.47 \pm 0.05$ & $14 \pm 2$ & $38 \pm 4$ \\
\hline & 1999 Feb 18 & 1.35 & $0.43 \pm 0.04$ & $11 \pm 2$ & $157 \pm 4$ \\
\hline & 1999 Apr 22 & 1.35 & $0.48 \pm 0.04$ & $9.3 \pm 2.5$ & $134 \pm 7$ \\
\hline & 1999 Sep 23 & 1.35 & $0.59 \pm 0.06$ & $9.7 \pm 2.5$ & $158 \pm 10$ \\
\hline & 2000 Jun 8 & 0.85 & $0.61 \pm 0.06$ & $9.6 \pm 1.3$ & $9 \pm 4$ \\
\hline & 2001 Jan 17 & 0.85 & $0.61 \pm 0.06$ & $11 \pm 2$ & $23 \pm 4$ \\
\hline & 2001 Jan 23 & 0.85 & $0.59 \pm 0.06$ & $14 \pm 3$ & $4 \pm 5$ \\
\hline \multirow[t]{10}{*}{ 3C $111 \ldots \ldots \ldots \ldots \ldots \ldots$} & 1998 Jul 17 & 1.35 & $1.03 \pm 0.10$ & $12 \pm 2$ & $39 \pm 4$ \\
\hline & 1998 Nov 30 & 1.35 & $1.45 \pm 0.10$ & $3.9 \pm 0.7$ & $117 \pm 5$ \\
\hline & 1998 Nov 23 & 1.35 & $1.26 \pm 0.13$ & $4.2 \pm 0.9$ & $123 \pm 6$ \\
\hline & 1998 Dec 13 & 1.35 & $1.37 \pm 0.14$ & $5.8 \pm 1.6$ & $39 \pm 7$ \\
\hline & 1999 Feb 18 & 1.35 & $1.04 \pm 0.10$ & $4.5 \pm 1.1$ & $126 \pm 6$ \\
\hline & 1999 Nov 23 & 1.35 & $0.89 \pm 0.09$ & $6.4 \pm 2.0$ & $143 \pm 11$ \\
\hline & 1999 Dec 24 & 0.85 & $0.93 \pm 0.10$ & $(4.9 \pm 1.9)$ & $(17 \pm 10)$ \\
\hline & 2000 Jun 8 & 0.85 & $1.05 \pm 0.11$ & $(1.0 \pm 1.5)$ & $(144 \pm 39)$ \\
\hline & $2001 \mathrm{Jan} 17$ & 0.85 & $3.61 \pm 0.36$ & $2.2 \pm 0.7$ & $46 \pm 8$ \\
\hline & 2001 Jan 23 & 0.85 & $5.99 \pm 0.60$ & $1.0 \pm 0.3$ & $17 \pm 7$ \\
\hline
\end{tabular}

Note.-Table 2 is published in its entirety in the electronic edition of the Astronomical Journal. A portion is shown here for guidance regarding its form and content.

unsubscribed telescope time. The data quality was quite variable, and typical total integration times were about 10 minutes per source. We omit from our analysis data taken during extremely high atmospheric phase variations or bad opacity conditions. We observed planets (Mars, Uranus, and Venus), $\mathrm{H}$ II regions (W3OH and MWC 349), and 3C 84 as quality checks and for flux calibration.

We analyzed the data by mapping all four Stokes parameters for each sideband separately, producing images of total intensity, linearly polarized flux density, degree of polarization, and polarization position angle for each source at each epoch. Data having $\mathrm{S} / \mathrm{N}<3$ for both the total and linearly polarized intensity images at the central pixel of the source were omitted. We determined the total and polarized flux densities by fitting a Gaussian brightness distribution to the corresponding image. Table 3 contains the results of the observations after correction of the degree of polarization for statistical bias; the columns are (1) source, (2) epoch of observation, (3) flux density $I_{3 \mathrm{~mm}}$, (4) degree of linear polarization $m_{3 \mathrm{~mm}}$, and (5) polarization position angle $\chi_{3 \mathrm{~mm}}$.

\section{4. $7 \mathrm{~mm}$ VLBA Observations}

We performed total and polarized intensity imaging at $43 \mathrm{GHz}$ with the Very Long Baseline Array (VLBA) at 17 epochs from 1998 March 25 to 2001 April 14. We describe the observations and data reduction in detail in $\mathrm{J} 05$, where the total and polarized intensity images are presented. Table 4 gives the results of fitting the VLBI core seen in the total and polarized intensity images by components with circular Gaussian brightness distributions (see J05). Columns of the table are as follows: (1) source, (2) epoch of observation, (3) flux density in the core $I_{7} \mathrm{~mm}$, (4) projected inner jet direction $\Theta_{\text {jet }},(5)$ degree of linear polarization in the core $m_{7 \mathrm{~mm}}$, and (6) polarization position angle in the core $\chi_{7 \mathrm{~mm}}$. We determine the projected inner jet direction from the position of the brightest jet component closest to, but at least one synthesized beam width from, the core.

Table 5 gives the polarization of jet components downstream of the VLBI core that are either brighter than or comparable to the core at least at one epoch $\left(I^{\text {comp }} \geq 0.5 I_{7} \mathrm{~mm}\right)$ or have detectable polarization at three or more epochs. These data allow us to probe the physics of the strongest disturbances that propagate down the jet. The columns of Table 5 are: (1) source, (2) epoch of observation, (3) designation of the component, which follows that of J05, (4) flux density in the component $I_{7 \mathrm{~mm}}^{\text {comp }}$, (5) distance from the core $R,(6)$ position angle relative to the core $\Theta,(7)$ degree

TABLE 3

BIMA Polarization Data

\begin{tabular}{|c|c|c|c|c|}
\hline $\begin{array}{c}\text { Source } \\
\text { (1) }\end{array}$ & $\begin{array}{c}\text { Epoch } \\
\text { (2) }\end{array}$ & $\begin{array}{c}I_{3 \mathrm{~mm}} \\
\text { (Jy) } \\
(3)\end{array}$ & $\begin{array}{c}m_{3 \mathrm{~mm}} \\
(\%) \\
(4)\end{array}$ & $\begin{array}{c}\chi_{3 \mathrm{~mm}} \\
(\mathrm{deg}) \\
(5)\end{array}$ \\
\hline \multirow[t]{10}{*}{ 3C 66A.......... } & 2000 Apr 21 & $0.82 \pm 0.01$ & $3.5 \pm 0.7$ & $171 \pm 5$ \\
\hline & 2000 Apr 25 & $0.98 \pm 0.01$ & $5.0 \pm 0.5$ & $1 \pm 2$ \\
\hline & 2001 Feb 6 & $0.89 \pm 0.01$ & $9.3 \pm 0.3$ & $17 \pm 1$ \\
\hline & 2001 Mar 20 & $0.65 \pm 0.01$ & $4.2 \pm 0.2$ & $2 \pm 1$ \\
\hline & 2001 Mar 29 & $0.85 \pm 0.01$ & $5.9 \pm 0.6$ & $10 \pm 2$ \\
\hline & 2001 Apr 3 & $0.86 \pm 0.01$ & $5.0 \pm 0.5$ & $8 \pm 2$ \\
\hline & 2001 Apr 7 & $0.71 \pm 0.01$ & $4.8 \pm 0.4$ & $17 \pm 2$ \\
\hline & 2001 Apr 12 & $0.90 \pm 0.01$ & $3.3 \pm 0.8$ & $32 \pm 6$ \\
\hline & 2001 Apr 16 & $0.71 \pm 0.01$ & $3.1 \pm 0.6$ & $0 \pm 5$ \\
\hline & 2001 Apr 20 & $1.00 \pm 0.02$ & $5.6 \pm 0.8$ & $15 \pm 4$ \\
\hline \multirow[t]{3}{*}{ 3C $111 \ldots \ldots \ldots$} & 2000 Apr 4 & $2.63 \pm 0.01$ & $0.3 \pm 0.1$ & $134 \pm 9$ \\
\hline & 2000 Apr 14 & $3.33 \pm 0.02$ & $2.0 \pm 1.2$ & $45 \pm 14$ \\
\hline & 2000 Dec 6 & $4.10 \pm 0.01$ & $0.5 \pm 0.2$ & $138 \pm 11$ \\
\hline
\end{tabular}

Note.-Table 3 is published in its entirety in the electronic edition of the Astronomical Journal. A portion is shown here for guidance regarding its form and content. 
TABLE 4

VlBA Polarization Data for the Core

\begin{tabular}{|c|c|c|c|c|c|}
\hline $\begin{array}{c}\text { Source } \\
\text { (1) }\end{array}$ & $\begin{array}{l}\text { Epoch } \\
\text { (2) }\end{array}$ & $\begin{array}{c}I_{7 \mathrm{~mm}} \\
(\mathrm{Jy}) \\
(3)\end{array}$ & $\begin{array}{c}\Theta_{\text {jet }} \\
(\mathrm{deg}) \\
(4)\end{array}$ & $\begin{array}{c}m_{7 \mathrm{~mm}} \\
(\%) \\
(5)\end{array}$ & $\begin{array}{c}\chi_{7 \mathrm{~mm}} \\
(\mathrm{deg}) \\
(6)\end{array}$ \\
\hline 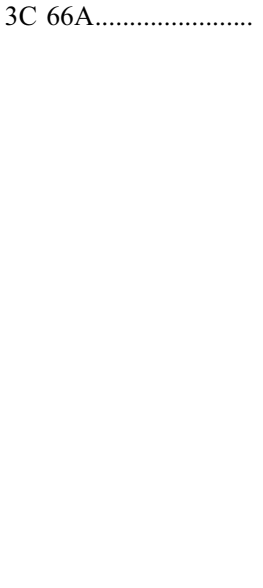 & $\begin{array}{l}\text { 1998 Mar } 25 \\
\text { 1998 May } 30 \\
\text { 1998 Jul } 31 \\
\text { 1998 Oct } 5 \\
\text { 1998 Dec } 10 \\
\text { 1999 Feb } 11 \\
\text { 1999 Apr } 29 \\
\text { 1999 Jul } 18 \\
\text { 1999 Oct } 6 \\
\text { 1999 Dec } 5 \\
\text { 2000 Jan } 24 \\
\text { 2000 Apr } 5 \\
\text { 2000 Jul } 17 \\
\text { 2000 Oct } 1 \\
\text { 2000 Dec } 11 \\
\text { 2001 Jan } 28 \\
\text { 2001 Apr } 14\end{array}$ & $\begin{array}{l}0.52 \pm 0.05 \\
0.45 \pm 0.07 \\
0.30 \pm 0.03 \\
0.31 \pm 0.04 \\
0.39 \pm 0.12 \\
0.48 \pm 0.05 \\
0.50 \pm 0.03 \\
0.47 \pm 0.02 \\
0.69 \pm 0.03 \\
0.75 \pm 0.03 \\
0.61 \pm 0.05 \\
0.60 \pm 0.05 \\
0.63 \pm 0.08 \\
0.73 \pm 0.04 \\
0.60 \pm 0.06 \\
0.63 \pm 0.07 \\
0.60 \pm 0.05\end{array}$ & $\begin{aligned}-146 & \pm 1 \\
-155 & \pm 1 \\
-150.7 & \pm 0.5 \\
-155 & \pm 1 \\
-159 & \pm 2 \\
-146 & \pm 2 \\
-167 & \pm 1 \\
-162 & \pm 2 \\
-154.4 & \pm 0.5 \\
-159 & \pm 1 \\
-155.4 & \pm 0.5 \\
-158 & \pm 1 \\
-163 & \pm 1 \\
-159 & \pm 1 \\
-160 & \pm 2 \\
-162 & \pm 2 \\
-160 & \pm 1\end{aligned}$ & $\begin{array}{l}2.3 \pm 0.6 \\
3.7 \pm 0.8 \\
2.9 \pm 0.6 \\
2.7 \pm 1.1 \\
3.1 \pm 1.3 \\
2.2 \pm 0.5 \\
1.4 \pm 0.6 \\
2.3 \pm 1.0 \\
3.2 \pm 0.4 \\
7.7 \pm 0.4 \\
3.9 \pm 0.4 \\
3.8 \pm 0.5 \\
4.3 \pm 1.7 \\
6.1 \pm 0.3 \\
5.7 \pm 1.0 \\
6.1 \pm 1.5 \\
5.4 \pm 0.3\end{array}$ & $\begin{aligned} 26 & \pm 7 \\
13 & \pm 5 \\
35 & \pm 6 \\
36 & \pm 10 \\
13 & \pm 11 \\
16 & \pm 6 \\
-6 & \pm 11 \\
27 & \pm 11 \\
18 & \pm 4 \\
5 & \pm 3 \\
0 & \pm 3 \\
14 & \pm 4 \\
28 & \pm 11 \\
28 & \pm 3 \\
27 & \pm 10 \\
35 & \pm 8 \\
28 & \pm 3\end{aligned}$ \\
\hline
\end{tabular}

Note.-Table 4 is published in its entirety in the electronic edition of the Astronomical Journal. A portion is shown here for guidance regarding its form and content.

TABLE 5

Vlba Polarization Data for the Jet Component

\begin{tabular}{|c|c|c|c|c|c|c|c|}
\hline $\begin{array}{c}\text { Source } \\
\text { (1) }\end{array}$ & $\begin{array}{l}\text { Epoch } \\
\text { (2) }\end{array}$ & $\begin{array}{c}\text { Component } \\
\text { (3) }\end{array}$ & $\begin{array}{c}I_{7 \mathrm{~mm}}^{\mathrm{comp}} \\
(\mathrm{Jy}) \\
(4)\end{array}$ & $\begin{array}{c}R \\
\text { (mas) } \\
(5)\end{array}$ & $\begin{array}{c}\Theta \\
(\mathrm{deg}) \\
(6)\end{array}$ & $\begin{array}{c}m_{7 \mathrm{~mm}}^{\text {comp }} \\
(\%) \\
(7)\end{array}$ & $\begin{array}{c}\chi_{7 \mathrm{~mm}}^{\text {comp }} \\
(\mathrm{deg}) \\
(8)\end{array}$ \\
\hline \multirow[t]{27}{*}{$3 \mathrm{C} 111 \ldots \ldots \ldots$} & \multirow[t]{2}{*}{1998 Mar 25} & $\mathrm{C} 1$ & $0.43 \pm 0.08$ & $2.50 \pm 0.10$ & $66.8 \pm 0.5$ & $6.3 \pm 2.1$ & $-44 \pm 10$ \\
\hline & & $\mathrm{c} 1$ & $0.14 \pm 0.06$ & $2.15 \pm 0.08$ & $67.6 \pm 0.5$ & $15.7 \pm 2.8$ & $-43 \pm 8$ \\
\hline & \multirow[t]{2}{*}{1998 May 30} & $\mathrm{C} 1$ & $0.54 \pm 0.08$ & $2.71 \pm 0.10$ & $67 \pm 1$ & $6.8 \pm 1.8$ & $-67 \pm 7$ \\
\hline & & $\mathrm{cl}$ & $0.10 \pm 0.04$ & $2.21 \pm 0.10$ & $69 \pm 1$ & $36 \pm 6$ & $-65 \pm 6$ \\
\hline & \multirow[t]{2}{*}{1998 Jul 31} & $\mathrm{C} 1$ & $0.32 \pm 0.08$ & $2.93 \pm 0.10$ & $67.7 \pm 0.5$ & $9.4 \pm 2.1$ & $-55 \pm 7$ \\
\hline & & $\mathrm{cl}$ & $0.16 \pm 0.05$ & $2.34 \pm 0.15$ & $68.5 \pm 0.5$ & $13 \pm 3$ & $-47 \pm 8$ \\
\hline & \multirow[t]{2}{*}{1998 Oct 5} & $\mathrm{C} 1$ & $0.22 \pm 0.06$ & $3.31 \pm 0.15$ & $67.5 \pm 1.0$ & $9.3 \pm 2.5$ & $-45 \pm 8$ \\
\hline & & $\mathrm{cl}$ & $0.09 \pm 0.04$ & $2.64 \pm 0.15$ & $70 \pm 2$ & $32 \pm 6$ & $-45 \pm 6$ \\
\hline & \multirow[t]{2}{*}{1998 Dec 10} & $\mathrm{C} 1$ & $0.17 \pm 0.05$ & $3.73 \pm 0.15$ & $67 \pm 1$ & $15 \pm 4$ & $-40 \pm 10$ \\
\hline & & $\mathrm{cl}$ & $0.16 \pm 0.04$ & $3.16 \pm 0.15$ & $69 \pm 2$ & $8.8 \pm 2.1$ & $-54 \pm 10$ \\
\hline & \multirow[t]{2}{*}{1999 Feb 11} & $\mathrm{C} 1$ & $0.17 \pm 0.05$ & $4.01 \pm 0.15$ & $67 \pm 1$ & $18.8 \pm 4.5$ & $-57 \pm 10$ \\
\hline & & $\mathrm{c} 1$ & $0.15 \pm 0.05$ & $3.41 \pm 0.15$ & $69 \pm 2$ & $8.8 \pm 2.1$ & $-60 \pm 10$ \\
\hline & \multirow[t]{2}{*}{1999 Apr 29} & $\mathrm{C} 1$ & $0.12 \pm 0.05$ & $4.33 \pm 0.20$ & $66 \pm 2$ & $21 \pm 5$ & $-22 \pm 20$ \\
\hline & & $\mathrm{c} 1$ & $0.16 \pm 0.07$ & $3.62 \pm 0.20$ & $70 \pm 2$ & $15.6 \pm 4.3$ & $-41 \pm 20$ \\
\hline & \multirow[t]{3}{*}{1999 Jul 18} & $\mathrm{C} 1$ & $0.20 \pm 0.05$ & $4.69 \pm 0.20$ & $66 \pm 2$ & $6.0 \pm 1.5$ & $-29 \pm 12$ \\
\hline & & $\mathrm{c} 2$ & $0.06 \pm 0.04$ & $4.11 \pm 0.20$ & $68 \pm 2$ & $37 \pm 7$ & $-31 \pm 20$ \\
\hline & & $\mathrm{cl}$ & $0.04 \pm 0.03$ & $3.69 \pm 0.20$ & $70 \pm 2$ & $38 \pm 8$ & $-26 \pm 20$ \\
\hline & \multirow[t]{3}{*}{1999 Oct 6} & $\mathrm{C} 1$ & $0.19 \pm 0.05$ & $5.16 \pm 0.15$ & $64 \pm 1$ & $8.9 \pm 1.6$ & $-27 \pm 8$ \\
\hline & & $\mathrm{c} 2$ & $0.07 \pm 0.03$ & $4.63 \pm 0.15$ & $67 \pm 1$ & $20.0 \pm 4.5$ & $-37 \pm 10$ \\
\hline & & $\mathrm{c} 1$ & $0.11 \pm 0.04$ & $3.90 \pm 0.15$ & $69 \pm 1$ & $10.9 \pm 3.4$ & $-31 \pm 10$ \\
\hline & \multirow[t]{3}{*}{1999 Dec 5} & $\mathrm{C} 1$ & $0.12 \pm 0.05$ & $5.46 \pm 0.15$ & $64 \pm 2$ & $14.5 \pm 3.6$ & $-28 \pm 10$ \\
\hline & & $\mathrm{c} 2$ & $0.06 \pm 0.03$ & $4.98 \pm 0.20$ & $66 \pm 2$ & $27 \pm 5$ & $-43 \pm 15$ \\
\hline & & $\mathrm{cl}$ & $0.10 \pm 0.04$ & $4.11 \pm 0.20$ & $68 \pm 2$ & $18.0 \pm 4.0$ & $-50 \pm 15$ \\
\hline & \multirow[t]{2}{*}{2000 Apr 5} & $\mathrm{c} 2$ & $0.05 \pm 0.03$ & $5.37 \pm 0.20$ & $64 \pm 2$ & $22 \pm 5$ & $-23 \pm 20$ \\
\hline & & $\mathrm{c} 1$ & $0.09 \pm 0.04$ & $4.52 \pm 0.20$ & $64 \pm 2$ & $15.6 \pm 4.5$ & $-42 \pm 20$ \\
\hline & \multirow[t]{2}{*}{$2000 \mathrm{Jul} 17$} & $\mathrm{c} 2$ & $0.05 \pm 0.03$ & $5.90 \pm 0.20$ & $64 \pm 2$ & $22.0 \pm 4.5$ & $-23 \pm 20$ \\
\hline & & $\mathrm{c} 1$ & $0.09 \pm 0.04$ & $4.69 \pm 0.20$ & $64 \pm 2$ & $13.3 \pm 4.0$ & $-42 \pm 20$ \\
\hline
\end{tabular}

Noте.-Table 5 is published in its entirety in the electronic edition of the Astronomical Journal. A portion is shown here for guidance regarding its form and content. 
of linear polarization $m_{7 \mathrm{~mm}}^{\text {comp }}$, and (8) polarization position angle $\chi_{7 \mathrm{~mm}}^{\text {comp }}$ in the component. Values of the degree of polarization in both tables are corrected for statistical bias.

\section{OBSERVED CHARACTERISTICS OF POLARIZATION}

Tables 1-4 show that the linear polarization for all objects at most wavelengths varies significantly both in degree and position angle. Figure 1 represents the relationship between the degree of polarization at optical, $1 \mathrm{~mm}$, and $3 \mathrm{~mm}$ wavelengths from the whole source with respect to the degree of polarization measured in the VLBI core at $7 \mathrm{~mm}$. The data plotted in Figure 1 correspond to the closest observations obtained at optical-7 $\mathrm{mm}, 1-7 \mathrm{~mm}$, and 3-7 mm wavelengths for each source. These measurements are simultaneous within 1-3 days. There is a statistically significant correlation at a significance level $\epsilon=0.05^{14}$ between fractional polarization in the VLBI core and degree of polarization at short wavelengths. The optical polarization maintains the strongest connection to the polarization in the VLBI core $(r=0.87$, where $r$ is the linear coefficient of correlation). This result confirms the strong correlation between the polarization level of the radio core at $7 \mathrm{~mm}$ and overall optical polarization found by Lister $\&$ Smith (2000). Their sample included quasars with both high and low optical polarization. However, it raises the question of why the optical polarization shows a better connection to the polarization in the VLBI core than does the polarization at $1 \mathrm{~mm}$.

\subsection{Polarization Variability}

We have computed the fractional polarization variability index $V_{\lambda}^{p}$ at each frequency using the definition employed by Aller et al. (2003b),

$$
V_{\lambda}^{p}=\frac{\left(m_{\lambda}^{\max }-\sigma_{m_{\lambda}^{\max }}\right)-\left(m_{\lambda}^{\min }+\sigma_{m_{\lambda}^{\min }}\right)}{\left(m_{\lambda}^{\max }-\sigma_{m_{\lambda}^{\max }}\right)+\left(m_{\lambda}^{\min }+\sigma_{m_{\lambda}^{\min }}\right)},
$$

where $m_{\lambda}^{\max }$ and $m_{\lambda}^{\min }$ are, respectively, the maximum and minimum fractional polarization measured over all epochs at wavelength $\lambda$, and $\sigma_{m}$ max and $\sigma_{m}$ min are the corresponding uncertainties. This approach is especially justified in our case, since all objects were observed over the same period of time, although the time intervals are different at different wavelengths $(\sim 2 \mathrm{yr}$ in the optical, $\sim 1$ yr at $3 \mathrm{~mm}$, and $\sim 3 \mathrm{yr}$ at 1 and $7 \mathrm{~mm}$ ). Note that equation (1) can produce a negative variability index if the degree of polarization or maximum change in polarization do not exceed the uncertainties in the measurements. In this case the data fail to reveal polarization variability, and we set $V^{p}=0$. We introduce the polarization position angle variability index, $V_{\lambda}^{a}$ :

$$
V_{\lambda}^{a}=\frac{\left|\Delta \chi_{\lambda}\right|-\sqrt{\sigma_{\chi_{\lambda}^{1}}^{2}+\sigma_{\chi_{\lambda}^{2}}^{2}}}{90}
$$

where $\Delta \chi_{\lambda}$ is the observed range of polarization direction and $\sigma_{\chi_{\lambda}^{1}}$ and $\sigma_{\chi_{\lambda}^{2}}$ are the uncertainties in the two values of EVPAs that define the range. We treat the $180^{\circ}$ ambiguities in EVPAs such that $\Delta \chi_{\lambda}$ cannot exceed $90^{\circ}$. As in the case of $V^{p}$, if $V^{a} \leq 0$ then we set $V^{a}=0$ and conclude that the observations were unable to measure variability in the polarization position angle.

\footnotetext{
14 Throughout the paper we calculate coefficients of linear correlation and use the method of Bowker \& Lieberman (1972) for testing the significance of these coefficients. The hypothesis that there is no correlation between two variables, $r=0$, can be rejected at a significance level $\epsilon$ if $t=\left|r /\left(1-r^{2}\right)^{1 / 2}\right|(N-2)^{1 / 2} \geq$ $t_{\epsilon / 2 ; N-2}$, where $t_{\epsilon / 2 ; N-2}$ is the percentage point of the $t$-distribution for $N-\overline{2}$ degrees of freedom and $N$ is the number of observations.
}

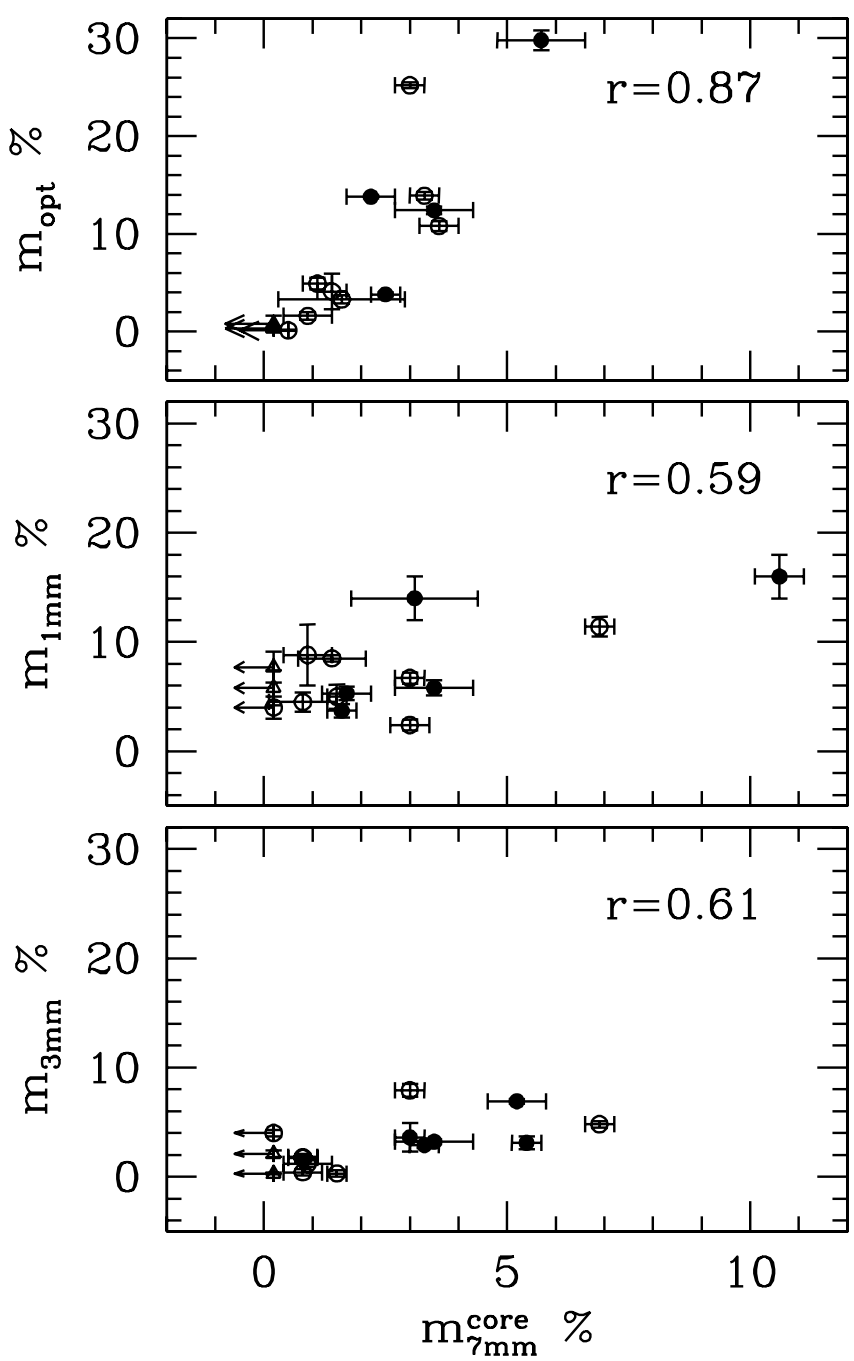

FIG. 1.-Degree of polarization at optical, $1 \mathrm{~mm}$, and $3 \mathrm{~mm}$ wavelengths from the whole source vs. degree of polarization measured in the VLBI core at $7 \mathrm{~mm}$ for the most nearly simultaneous pair of observations for each source. The linear coefficient of correlation, $r$, is given in each panel. Symbols denote quasars (open circles), BL Lac objects (filled circles), and radio galaxies (triangles).

Figure 2 shows the relationship between the polarization and position angle variability indices in the VLBI core and at short wavelengths. A statistically significant correlation occurs both between $V_{\text {opt }}^{p}$ and $V_{7 \mathrm{~mm}}^{p}$ and between $V_{\mathrm{opt}}^{a}$ and $V_{7 \mathrm{~mm}}^{a}(r=0.62$ and 0.78 , respectively). This result indicates that changes in the ordering of magnetic fields in the optical region and VLBI core are correlated, which suggests that the polarization variability has the same origin at the two wave bands. The correlation between the values at $3 \mathrm{~mm}$ and in the VLBI core might be affected by the region of the jet lying somewhat outside the VLBI core that contributes significantly to the $3 \mathrm{~mm}$ polarized emission. There is no correlation between variability indices at $1 \mathrm{~mm}$ and in the VLBI core.

\subsection{A Polarization-based Classification Scheme}

We use the polarization variability indices to classify the sources in our sample with respect to their polarization properties. This classification scheme differs somewhat from the classical separation of AGNs into radio galaxies, quasars, and BL Lac objects, which we have used to discuss kinematics in the parsec-scale jets (J05). The new categorization reveals significant differences in 

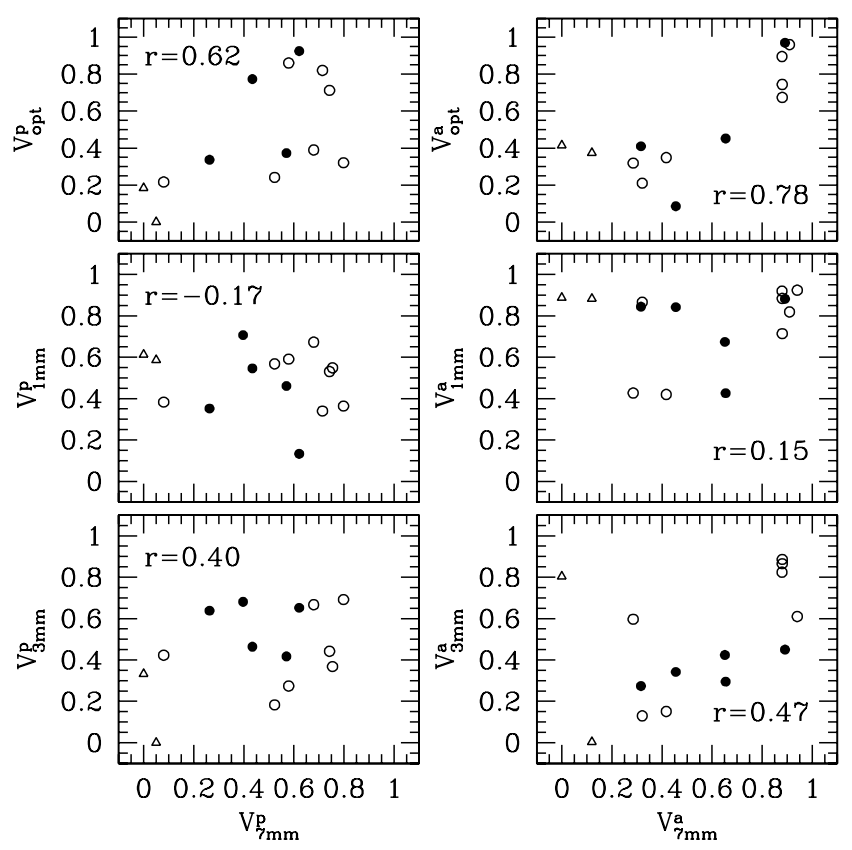

FIG. 2.-Left: Dependence on polarization variability index at optical, $1 \mathrm{~mm}$, and $3 \mathrm{~mm}$ wavelengths and polarization variability index in the VLBI core at $7 \mathrm{~mm}$. Right: Dependence on polarization position angle variability index at optical, $1 \mathrm{~mm}$, and $3 \mathrm{~mm}$ wavelengths and polarization position angle variability index in the VLBI core at $7 \mathrm{~mm}$. The symbols are the same as in Fig. 1.

the polarization properties (see $\S 7$ ) among the introduced groups that would be diluted in the traditional scheme.

Figure 3 shows that the fractional polarization and polarization position angle variability indices in the $7 \mathrm{~mm}$ core are strongly correlated $(r=0.83)$ in a way that produces a clear separation of the sources into three groups: LVP (low variability of polarization in the radio core, $V_{7 \mathrm{~mm}}^{p} \leq 0.2, V_{7 \mathrm{~mm}}^{a} \leq 0.4$ ), IVP (intermediate variability of polarization, $0.2<V_{7 \mathrm{~mm}}^{p} \leq 0.6,0.2<V_{7 \mathrm{~mm}}^{a} \leq$ 0.7 ), and HVP (high variability of polarization in the radio core, $V_{7 \mathrm{~mm}}^{p}>0.6, V_{7 \mathrm{~mm}}^{a}>0.7$ ). (We note, however, that low electric vector variability indices in the LVP group could result from large uncertainties in EVPAs connected with low fractional polarization.)

The LVP group includes the two radio galaxies 3C 111 and $3 \mathrm{C} 120$ plus the low optically polarized quasar $3 \mathrm{C} 273$. All three sources display low fractional polarization in the optical band and in the $7 \mathrm{~mm}$ core. However, in the quasar 3C 273 the optical synchrotron emission is known to be variable and to have $m>$ $10 \%$ if dilution from essentially unpolarized nonsynchrotron components such as the big blue bump is taken into account (Impey et al. 1989). All three LVP sources are highly polarized and variable at $1 \mathrm{~mm}$, and the fractional polarization of $3 \mathrm{C} 273$ at $3 \mathrm{~mm}$ might be as high as $4 \%$.

The IVP group consists of four (out of five) BL Lac objects and two highly optically polarized quasars, 3C 279 and 3C 345. This group, therefore, includes extremely highly polarized blazars, whose polarization in the optical and at $1 \mathrm{~mm}$ can exceed $30 \%$ and whose polarization at $3 \mathrm{~mm}$ and in the VLBI core does not drop below $2 \%-3 \%$. Note that Nartallo et al. (1998) have found no differences in polarization properties of BL Lac objects and compact flat-spectrum quasars at short millimeter and submillimeter wavelengths.

The majority of the quasars (five out of eight) and one BL Lac object, OJ 287, form the HVP group. The linear polarization of the VLBI core in these sources can be very low, i.e., at the noise level, or as high as $\geq 5 \%$. The polarization variability indices, $V^{p}$, at $3 \mathrm{~mm}, 1 \mathrm{~mm}$, and optical wavelengths are similar to those

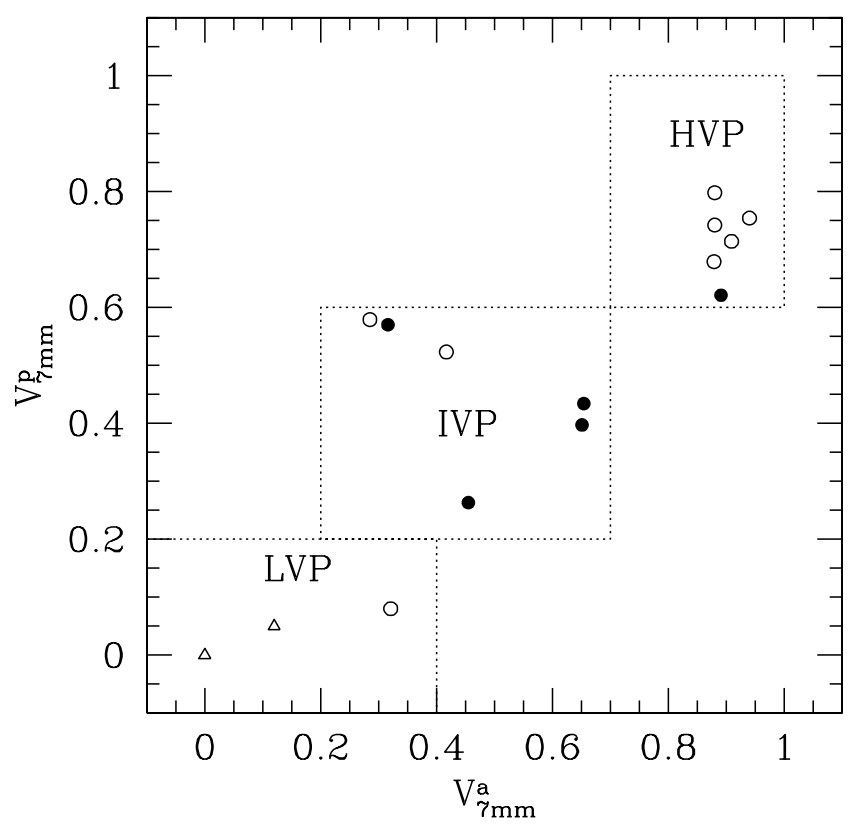

FIG. 3.-Connection between polarization and position angle variability indices in the VLBI core at $7 \mathrm{~mm}$. Symbols denote the quasars (open circles), BL Lac objects ( filled circles), and radio galaxies (triangles).

for the IVP group, although $V^{a}$ indices indicate more dramatic polarization position angle variability at all wavelengths. We did not observe high polarization at short millimeter wavelengths $\left(m_{3 \mathrm{~mm}} \geq 10 \%\right.$ and $\left.m_{1 \mathrm{~mm}} \geq 15 \%\right)$ as in the IVP group, although very high optical polarization $\left(m_{\mathrm{opt}} \geq 20 \%\right)$ might occur in these sources as often as in IVP sources (Hagen-Thorn 1980).

Figure 4 presents the distributions of fractional polarization for all of our measurements, separated according to the variability groups. Comparison of the distributions shows that (1) the peak of the distribution in the IVP group occurs at a higher percentage polarization than for the other two groups, independent of wavelength, and (2) for a given variability group, the peak of the distribution shifts to higher fractional polarization at shorter wavelengths except for the LVP sources, where the optical polarization is similar to the polarization in the VLBI core.

\section{TOTAL AND POLARIZED FLUX DENSITY SPECTRA}

We have constructed single-epoch total and polarized flux density spectra based on measurements at different wavelengths obtained within 2 weeks of each other (except 1803+784 and $1823+568$, where, at best, there is nearly a month between observations at different wavelengths). We have corrected the optical flux densities shown in Table 1 for Galactic extinction using values of $A_{\lambda}$ compiled by the NASA/IPAC Extragalactic Database. The total flux densities at $7 \mathrm{~mm}$ are corrected for possible missing flux density in the VLBA images using single-dish observations as described in J05. The measurements at $7 \mathrm{~mm}$ include the core and components within the $1 \%$ contours of the peak total intensity at a given epoch (J05), $I_{\mathrm{jet}}=I^{\text {core }}+\sum_{i} I_{i}^{\text {comp }}$. The polarized flux density at $7 \mathrm{~mm}$ is integrated over the same VLBA image. Therefore, $I_{\text {jet }}^{p}=\left(Q_{\text {jet }}^{2}+U_{\text {jet }}^{2}\right)^{1 / 2}, Q_{\text {jet }}=Q^{\text {core }}+\sum_{i} Q_{i}^{\text {comp }}, U_{\text {jet }}=$ $U^{\text {core }}+\sum_{i} U_{i}^{\text {comp },}, m_{\text {jet }}=I_{\text {jet }}^{p} / I_{\text {jet }}$, and $\chi_{\text {jet }}=0.5 \tan ^{-1}\left(U_{\text {jet }} / Q_{\text {jet }}\right)$, where $I^{\text {core }}, Q^{\text {core }}$, and $U^{\text {core }}$ are the Stokes parameters of the core, and $I_{i}^{\text {comp }}, Q_{i}^{\text {comp }}$, and $U_{i}^{\text {comp }}$ are the Stokes parameters of a given polarized jet component. Figure 5 presents the total and polarized spectral energy distributions (SEDs), while Table 6 lists the spectral indices $\alpha_{\mathrm{mm}}$ (based on total flux densities at the three millimeter 


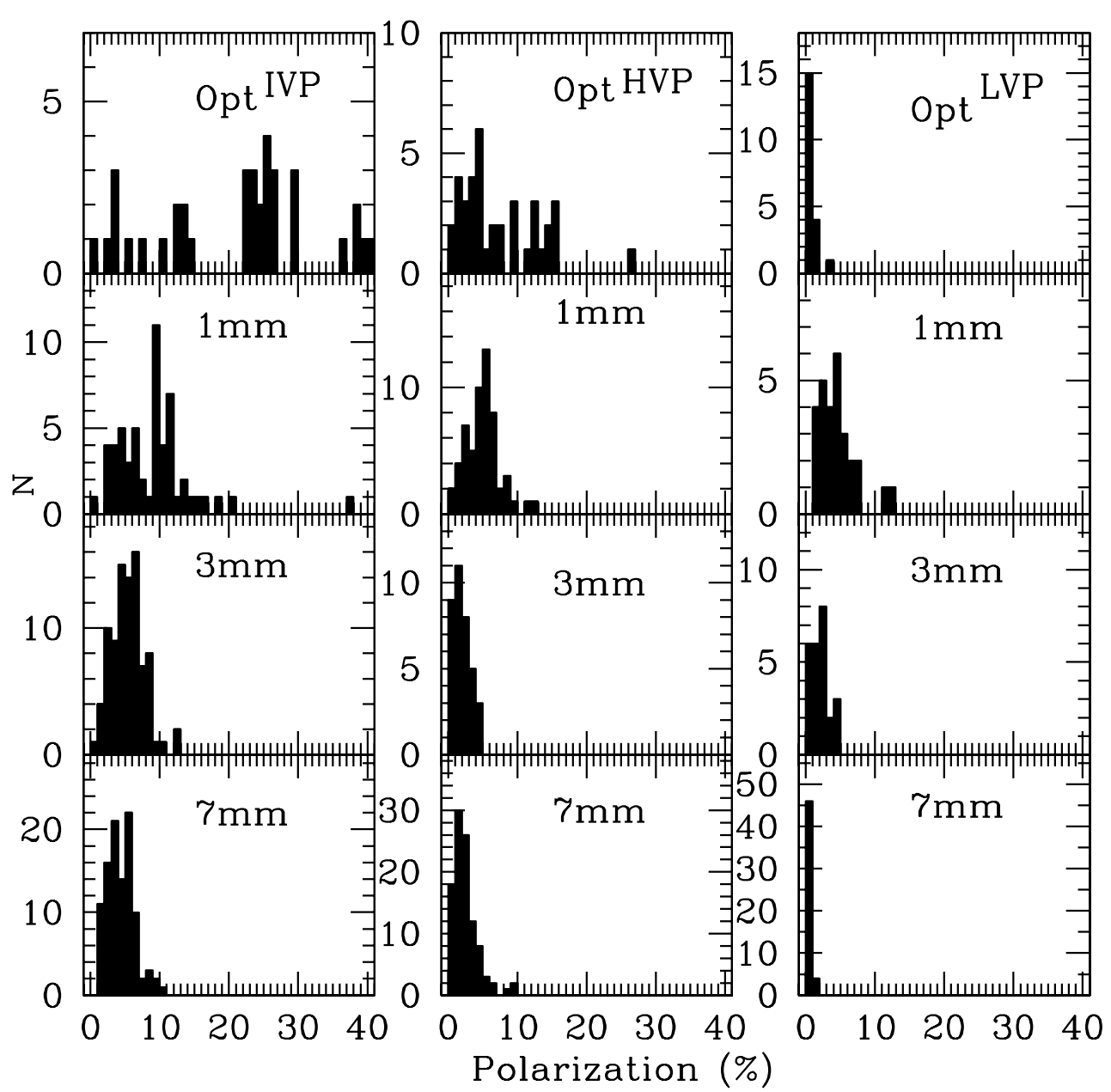

FIG. 4.-Distributions of degree of linear polarization in the IVP (left), HVP (middle), and LVP (right) variability groups at optical, $1 \mathrm{~mm}$, and $3 \mathrm{~mm}$ wavelengths and in the VLBI core at $7 \mathrm{~mm}$.

wavelengths) and $\alpha_{\mathrm{opt} / 1 \mathrm{~mm}}$, where $S_{\nu} \propto \nu^{-\alpha}$. We calculate the spectral indices $\alpha_{\mathrm{mm}}^{p}$ and $\alpha_{\mathrm{opt} / 1 \mathrm{~mm}}^{p}$ using the corresponding polarized flux densities, $S_{\nu}^{p} \propto \nu^{-\alpha^{p}}$.

Table 6 shows that the total flux density spectra at millimeter wavelengths are flat independent of the type of source, with the majority of spectral indices having $\left|\alpha_{\mathrm{mm}}\right|<0.5$. This confirms that the millimeter-wave emission is partially optically thick. The optical-millimeter spectra of the HVP sources are steep, with $\alpha_{\text {opt } / 1 \mathrm{~mm}} \sim 1$, while the LVP sources possess much flatter optical to $1 \mathrm{~mm}$ spectra. In the IVP group $\alpha_{\text {opt } / 1 \mathrm{~mm}}$ depends on whether the source is a quasar $\left(\alpha_{\text {opt } / 1 \mathrm{~mm}} \sim 1\right)$ or a BL Lac object $\left(\alpha_{\text {opt } / 1 \mathrm{~mm}} \sim 0.5\right)$. The polarized flux density spectra at millimeter wavelengths are flat or inverted, which indicates an increase of polarized emission with frequency at millimeter wavelengths. Figure 6 reveals a strong correlation between $\alpha_{\mathrm{opt} / 1 \mathrm{~mm}}^{p}$ and $\alpha_{\mathrm{opt} / 1 \mathrm{~mm}}(r=0.92)$. However, two sources from the LVP group (3C 120 and 3C 273) deviate greatly from the dependence $\alpha_{\mathrm{opt} / 1 \mathrm{~mm}}^{p}=\alpha_{\mathrm{opt} / 1 \mathrm{~mm}}$ (Fig. 6, solid line), with a significantly steeper polarized flux density spectral index than for the total flux density. This suggests that at least two emission components are present in the optical region, one of which is unpolarized. Impey et al. (1989) found an increase in the degree of polarization and higher variability of the Stokes parameters in 3C 273 at longer optical wavelengths. Such behavior is expected if the optical emission consists of a variable synchrotron component plus a relatively static blue unpolarized continuum source, such as the big blue bump. The decomposition of the optical syn- chrotron spectrum from other optical emission sources is readily seen in the spectropolarimetry of 3C 273 (Smith et al. 1993). In the case of $3 \mathrm{C} 120$, the strong dilution of the optical synchrotron polarization is likely caused by host galaxy starlight included within the measurement aperture.

In the majority of the HVP and IVP sources, the spectral indices $\alpha_{\mathrm{opt} / 1 \mathrm{~mm}}^{p}$ and $\alpha_{\mathrm{opt} / 1 \mathrm{~mm}}$ are similar to each other. This suggests that a single synchrotron component is responsible for the total flux and polarized continuum from millimeter to optical wavelengths. However, three of the HVP sources (0420-014, 1510-089, and 3C 454.3) have a slightly steeper polarized flux density spectrum relative to the total flux density spectrum (this was found previously as well by Smith et al. 1988). In the IVP sources the opposite trend prevails, except for BL Lac. A steeper polarized spectrum in the quasars indicates the possible presence of a nonsynchrotron component, such as that observed for $3 \mathrm{C} 273$, although its contribution to the total flux should be $\$ 10 \%$ relative to the synchrotron emission. A flatter polarized spectrum likely rules out any significant contribution by a nonsynchrotron component, although two (or more) synchrotron components might coexist in the IVP sources. For BL Lac, contribution to the optical flux from the host galaxy might help to explain why the $\alpha_{\mathrm{opt} / 1 \mathrm{~mm}}^{p}$ is slightly steeper than the total flux spectral index.

\section{FARADAY ROTATION IN THE INNER JET}

Figure 5 presents measurements of the polarization angle $\chi$ at different frequencies obtained within 2 weeks of each other and 

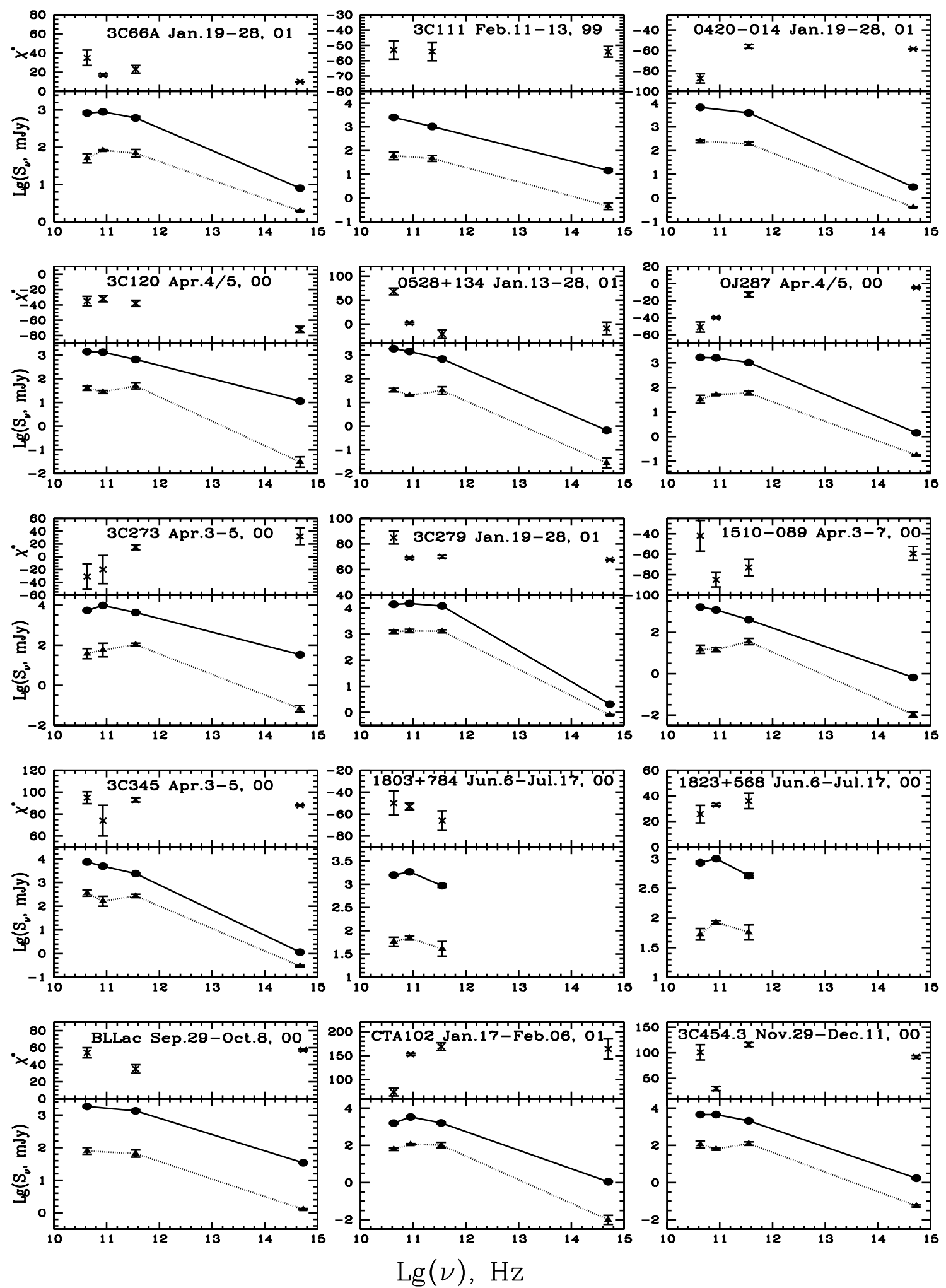

Fig. 5.-Total (circles) and polarized (triangles) flux density spectra and polarization position angle (crosses) measurements from optical to $7 \mathrm{~mm}$ wavelengths.

shows that the direction of millimeter-wave polarization rotates with wavelength. In the HVP and IVP sources the EVPA at $7 \mathrm{~mm}$ displayed in the figure corresponds to that of the VLBI core only, while in the LVP sources it corresponds to $\chi_{\text {jet }}$, as described in $\S 4$. We attribute this rotation to Faraday rotation by a foreground screen close to the VLBI core (Zavala \& Taylor 2004; Attridge et al. 2005). We define the rotation measure (RM) by assuming that at $1 \mathrm{~mm}$ we see the unrotated direction of the polarization and that the emission from 1 to $7 \mathrm{~mm}$ propagates through the same screen.

\subsection{Faraday Rotation in the HVP and IVP Sources}

Figure 7 shows the result of fitting the dependence of the polarization position angle on wavelength by a Faraday rotation law: 
TABLE 6

Total and Polarized Flux Spectral Indices

\begin{tabular}{|c|c|c|c|c|c|}
\hline $\begin{array}{l}\text { Source } \\
\text { (1) }\end{array}$ & $\begin{array}{l}\text { Group } \\
\text { (2) }\end{array}$ & $\begin{array}{c}\alpha_{\mathrm{opt} / 1 \mathrm{~mm}} \\
\text { (3) }\end{array}$ & $\begin{array}{c}\alpha_{\mathrm{opt} / 1 \mathrm{~mm}}^{p} \\
\text { (4) }\end{array}$ & $\begin{array}{c}\alpha_{\mathrm{mm}} \\
(5)\end{array}$ & $\begin{array}{l}\alpha_{\mathrm{mm}}^{p} \\
(6)\end{array}$ \\
\hline 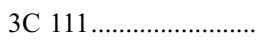 & LVP & $0.55 \pm 0.02$ & $0.60 \pm 0.04$ & $0.52 \pm 0.05$ & $0.15 \pm 0.20$ \\
\hline $3 \mathrm{C} 120$ & LVP & $0.56 \pm 0.02$ & $1.03 \pm 0.05$ & $0.47 \pm 0.02$ & $-0.28 \pm 0.34$ \\
\hline 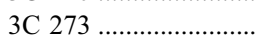 & LVP & $0.66 \pm 0.01$ & $1.04 \pm 0.07$ & $0.64 \pm 0.16$ & $0.51 \pm 0.27$ \\
\hline 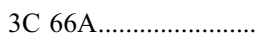 & IVP & $0.60 \pm 0.01$ & $0.50 \pm 0.02$ & $0.16 \pm 0.09$ & $-0.11 \pm 0.21$ \\
\hline 3C 279 & IVP & $1.19 \pm 0.01$ & $1.01 \pm 0.04$ & $0.08 \pm 0.06$ & $-0.13 \pm 0.03$ \\
\hline 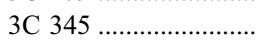 & IVP & $1.06 \pm 0.01$ & $0.95 \pm 0.01$ & $0.53 \pm 0.06$ & $0.06 \pm 0.36$ \\
\hline $1803+784 \ldots \ldots \ldots \ldots \ldots \ldots \ldots$ & IVP & $\ldots$ & $\ldots$ & $0.28 \pm 0.17$ & $0.19 \pm 0.15$ \\
\hline $1823+568 \ldots \ldots \ldots \ldots \ldots \ldots$ & IVP & $\ldots$ & $\ldots$ & $0.27 \pm 0.17$ & $0.12 \pm 0.23$ \\
\hline 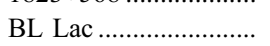 & IVP & $0.50 \pm 0.01$ & $0.54 \pm 0.02$ & $0.15 \pm 0.04$ & $0.08 \pm 0.09$ \\
\hline 0420-014 …................... & HVP & $1.00 \pm 0.01$ & $0.86 \pm 0.01$ & $0.25 \pm 0.03$ & $0.11 \pm 0.05$ \\
\hline 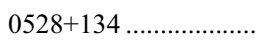 & HVP & $0.96 \pm 0.02$ & $0.98 \pm 0.05$ & $0.48 \pm 0.03$ & $-0.32 \pm 0.26$ \\
\hline 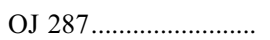 & HVP & $0.90 \pm 0.01$ & $0.80 \pm 0.01$ & $0.24 \pm 0.06$ & $-0.25 \pm 0.12$ \\
\hline $1510-089$...................... & HVP & $0.90 \pm 0.01$ & $1.13 \pm 0.04$ & $0.68 \pm 0.07$ & $-0.44 \pm 0.17$ \\
\hline 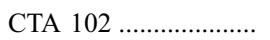 & HVP & $1.00 \pm 0.02$ & $0.97 \pm 0.06$ & $0.37 \pm 0.20$ & $0.11 \pm 0.12$ \\
\hline 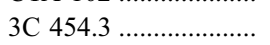 & HVP & $0.97 \pm 0.01$ & $1.05 \pm 0.02$ & $0.40 \pm 0.13$ & $-0.11 \pm 0.32$ \\
\hline
\end{tabular}

Notes.-Uncertainties for $\alpha_{\mathrm{opt} / 1 \mathrm{~mm}}$ are determined from uncertainties of flux measurements; uncertainties for $\alpha_{\mathrm{mm}}$ are based on consistency between different wavelengths.

$\chi=\chi_{1 \mathrm{~mm}}+\mathrm{RM} \lambda^{2}$. For 0420-014 our program does not contain simultaneous observations at three wavelengths necessary to estimate RM. We have supplemented our data by observations obtained with the VLBA at $7 \mathrm{~mm}$ and $1.3 \mathrm{~cm}$ in the program BG073 (see Gómez et al. 2000), for which 0420-014 and 3C 454.3 were used as calibrators. For $0420-014$ we use our polarization measurement at $1 \mathrm{~mm}$ on 1998 December 13 and EVPAs in the $7 \mathrm{~mm}$ and $1.3 \mathrm{~cm}$ core on 1998 December 3 from BG073. The EVPA at $7 \mathrm{~mm}$ is consistent with $\chi_{7 \mathrm{~mm}}$ at epoch 1998 December 11 from our program. For 3C 454.3 and BL Lac we use observations different from those plotted in Figure 5. For $3 \mathrm{C} 454.3$ we use observations at 1 and $3 \mathrm{~mm}$ from our program (2000 June 6 and 8, respectively) and $7 \mathrm{~mm}$ VLBA observations on 2000 June 9 (BG073). These observations are closer to each other than the measurements presented in Figure 5 (2000

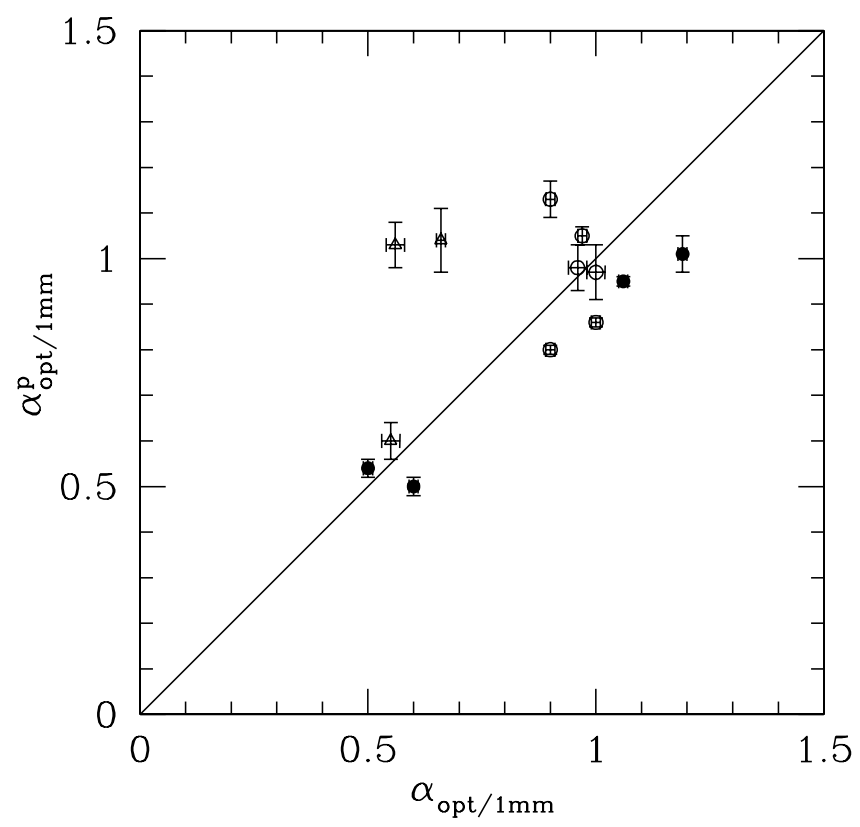

FIG. 6.-Dependence between polarized and total flux spectral indices calculated between the optical and $1 \mathrm{~mm}$ wavelengths (open circles: HVP sources; filled circles: IVP sources; triangles: LVP sources).
November 29-December 11) and less affected by the variability of bright component B6 seen on the images (J05). We have included the contribution of the component in the polarization at $7 \mathrm{~mm}$ because B6 is brighter and more highly polarized than the core and located within 0.2 mas of the core. For BL Lac we use observations carried out over the period 2001 January 17-28 in order to have simultaneous measurements at $3 \mathrm{~mm}$ wavelengths.

Table 7 gives the values of correction for EVPAs at 3 and $7 \mathrm{~mm}$ along with the intrinsic rotation measure for each object, $\mathrm{RM}^{0}=$ $\mathrm{RM}(1+z)^{2}$. Some of the RM values have large uncertainties that might be attributed to several problems that our observations possess. First, the observations are not completely simultaneous, whereas the polarization position angle can sometimes have short timescales of variability even at $7 \mathrm{~mm}$ (D'Arcangelo et al. 2007). Second, the polarization position angles at $7 \mathrm{~mm}$ correspond to those of the VLBI core only, while the EVPAs at 1 and at $3 \mathrm{~mm}$ are from the whole source. Although we have tested that, at epochs used for estimates of the rotation measure, jet components contribute little to the polarization of the core (except 3C 454.3), their contribution to the polarized flux at 1 and $3 \mathrm{~mm}$ is unknown. Third, the structure of the magnetic field in the $7 \mathrm{~mm}$ core and in the regions emitting at shorter millimeter wavelengths, especially at $1 \mathrm{~mm}$, might be different. Therefore, the observed rotation could be an intrinsic property of the magnetic field structure.

To test that the derived rotation measures are reliable, we construct the distributions of $\Delta \chi_{3-7}=\left|\chi_{3 \mathrm{~mm}}-\chi_{7 \mathrm{~mm}}\right|$ for all pairs of observations simultaneous within 2 weeks for $\chi_{\lambda}$ before and after RM correction (Fig. 8). Note that the distributions do not include the observations used to calculate the rotation measures. This avoids the bias that these observations would introduce to the distributions. Ideally, the EVPAs after RM correction should align within $10^{\circ}-20^{\circ}$, which corresponds to the uncertainties of our measurements. This is a feature of both distributions (uncorrected and corrected) for the IVP sources, reflecting the fact that in the IVP sources the uncertainties of EVPAs are comparable to the RM correction values. However, EVPAs corrected for RM exhibit a slightly better alignment than $\Delta \chi_{3-7}$ before the correction, as is expected if the EVPA rotation is caused by a Faraday screen. In the HVP sources the RM correction significantly improves alignment between $\chi_{3 \mathrm{~mm}}$ and $\chi_{7 \mathrm{~mm}} ; 69 \%$ of uncorrected values of $\Delta \chi_{3-7}$ exceed $20^{\circ}$, while only $44 \%$ of 

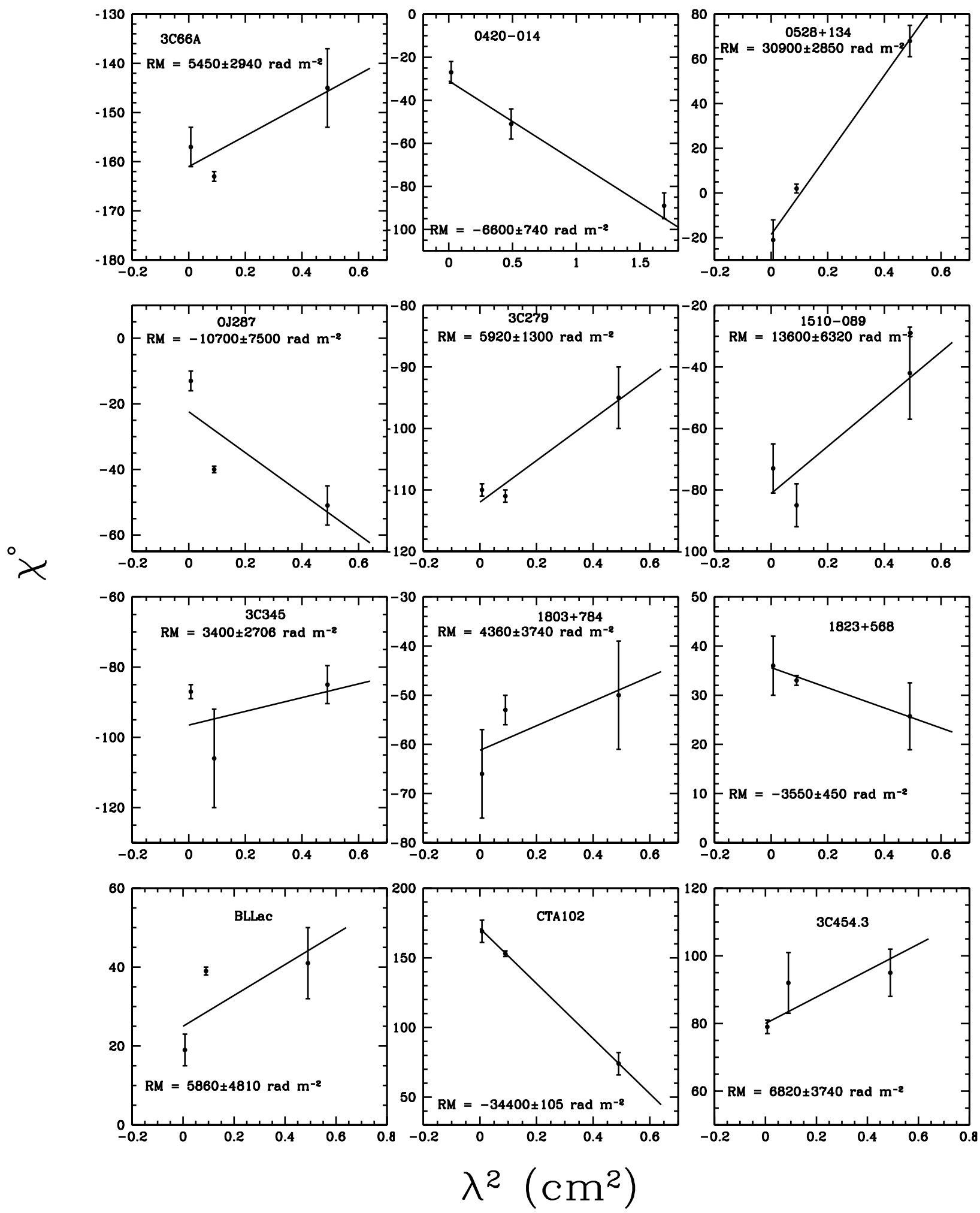

FIG. 7.-Dependence of polarization position angle on square of wavelength in the IVP and HVP sources. The solid line represents an approximation of the dependence by a $\lambda^{2}$ Faraday rotation law.

corrected EVPAs have $\Delta \chi_{3-7}>20^{\circ}$. The existence of a few large misalignments between EVPAs after the RM correction can be attributed to variability of RM in the VLBI core of quasars (Zavala \& Taylor 2004). This partially affects the analysis performed in $\S 7$.

Table 7 shows that the IVP sources have lower RMs, $\left\langle\left|\mathrm{RM}^{0}\right|\right\rangle=$ $(1.1 \pm 0.3) \times 10^{4} \mathrm{rad} \mathrm{m}^{-2}$, than the HVP sources, $\left\langle\left|\mathrm{RM}^{0}\right|\right\rangle=$
$(8.5 \pm 6.5) \times 10^{4} \mathrm{rad} \mathrm{m}^{-2}$. Two of the HVP blazars, $0528+128$ and CTA 102, have EVPA corrections at $7 \mathrm{~mm}$ close to $90^{\circ}$. A $90^{\circ}$ flip in EVPA can be caused by the spectral properties of the source-optically thin at $1 \mathrm{~mm}$ but optically thick at $7 \mathrm{~mm}$. However, according to Pacholczyk (1970) the degree of polarization should decrease significantly (by a factor of $\sim 7$ ) when the optical depth to synchrotron self-absorption exceeds unity, while both 
TABLE 7

Rotation Measures

\begin{tabular}{|c|c|c|c|c|c|c|c|c|}
\hline $\begin{array}{c}\text { Source } \\
\text { (1) }\end{array}$ & $\begin{array}{c}z \\
(2)\end{array}$ & $\begin{array}{l}\text { Group } \\
\text { (3) }\end{array}$ & $\begin{array}{c}\Delta \chi_{3 \mathrm{~mm}} \\
(\mathrm{deg}) \\
\quad(4)\end{array}$ & $\begin{array}{l}\Delta \chi_{7 \mathrm{~mm}} \\
(\mathrm{deg}) \\
\quad(5)\end{array}$ & $\begin{array}{c}\mathrm{RM}_{43 \mathrm{GHz}}^{0} \\
\left(10^{3} \mathrm{rad} \mathrm{m}^{-2}\right) \\
(6)\end{array}$ & $\begin{array}{c}\mathrm{RM}_{15 \mathrm{GHz}}^{0} \\
\left(10^{3} \mathrm{rad} \mathrm{m}^{-2}\right) \\
(7)\end{array}$ & $\begin{array}{c}\mathrm{RM}_{8 \mathrm{GHz}}^{0} \\
\left(10^{3} \mathrm{rad} \mathrm{m}^{-2}\right) \\
(8)\end{array}$ & $\begin{array}{c}a \\
(9)\end{array}$ \\
\hline 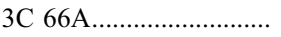 & 0.444 & IVP & -3 & -16 & $12 \pm 6$ & $\ldots$ & $\ldots$ & $\ldots$ \\
\hline 3С $279 \ldots \ldots \ldots \ldots \ldots \ldots \ldots \ldots$ & 0.538 & IVP & -3 & -16 & $14 \pm 2$ & $-5.7(2)$ & $3.1(1)$ & $0.92 \pm 0.04$ \\
\hline 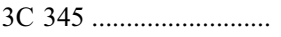 & 0.595 & IVP & -2 & -9 & $11 \pm 7$ & $\ldots$ & $-0.33^{*}(3)$ & 1.6 \\
\hline $1803+784 \ldots \ldots \ldots \ldots \ldots \ldots \ldots$ & 0.68 & IVP & -3 & -16 & $12 \pm 9$ & $\ldots$ & $0.56(1)$ & 1.8 \\
\hline $1823+568 \ldots \ldots \ldots \ldots \ldots \ldots \ldots$ & 0.664 & IVP & 1 & 7 & $-10 \pm 1$ & $1.1(2)$ & $0.36(1)$ & $1.81 \pm 0.02$ \\
\hline BL Lac ............................ & 0.069 & IVP & -3 & -16 & $6.7 \pm 5.5$ & $7.0(2)$ & $0.42(1)$ & $1.5 \pm 1.2$ \\
\hline $0420-014 \ldots \ldots \ldots \ldots \ldots \ldots \ldots$ & 0.915 & HVP & 4 & 19 & $-24 \pm 3$ & $\ldots$ & $\ldots$ & $\ldots$ \\
\hline 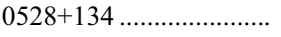 & 2.06 & HVP & -16 & -87 & $280 \pm 30$ & $\cdots$ & $1.5(1)$ & 3.1 \\
\hline 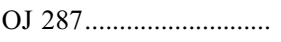 & 0.306 & HVP & 6 & 30 & $-19 \pm 11$ & $\cdots$ & $\ldots$ & $\cdots$ \\
\hline $1510-089 \ldots \ldots \ldots \ldots \ldots \ldots \ldots$ & 0.361 & HVP & -7 & -38 & $26 \pm 10$ & $\ldots$ & $\ldots$ & $\ldots$ \\
\hline CTA $102 \ldots \ldots \ldots \ldots \ldots \ldots \ldots$ & 1.037 & HVP & 18 & 97 & $-140 \pm 40$ & $\ldots$ & $-4.2^{*}(4)$ & 1.6 \\
\hline 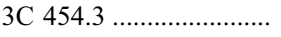 & 0.859 & HVP & -4 & -19 & $24 \pm 13$ & $\ldots$ & $9.0(1)$ & 1.8 \\
\hline
\end{tabular}

Notes.-Col. (1): Name of the source. Col. (2): Redshift. Col. (3): Polarization variability group. Col. (4): RM correction at $3 \mathrm{~mm}$. Col. (5): RM correction at $7 \mathrm{~mm}$. Col. (6): Intrinsic RM obtained in the $43 \mathrm{GHz}$ core. Col. (7): Intrinsic RM obtained in the $15 \mathrm{GHz}$ core. Col. (8): Intrinsic RM obtained in the $8 \mathrm{GHz}$ core. An asterisk denotes intrinsic RM obtained in the core at $5 \mathrm{GHz}$ instead of $8 \mathrm{GHz}$; numbers in parentheses denote references for $\mathrm{RM}^{0}$ measured at low frequencies $(1:$ Zavala \& Taylor 2003; 2: Gabuzda et al. 2006; 3: Taylor 1998; 4: Taylor 2000). Col. (9): The exponent in the relation RM $\propto d^{-a}$.

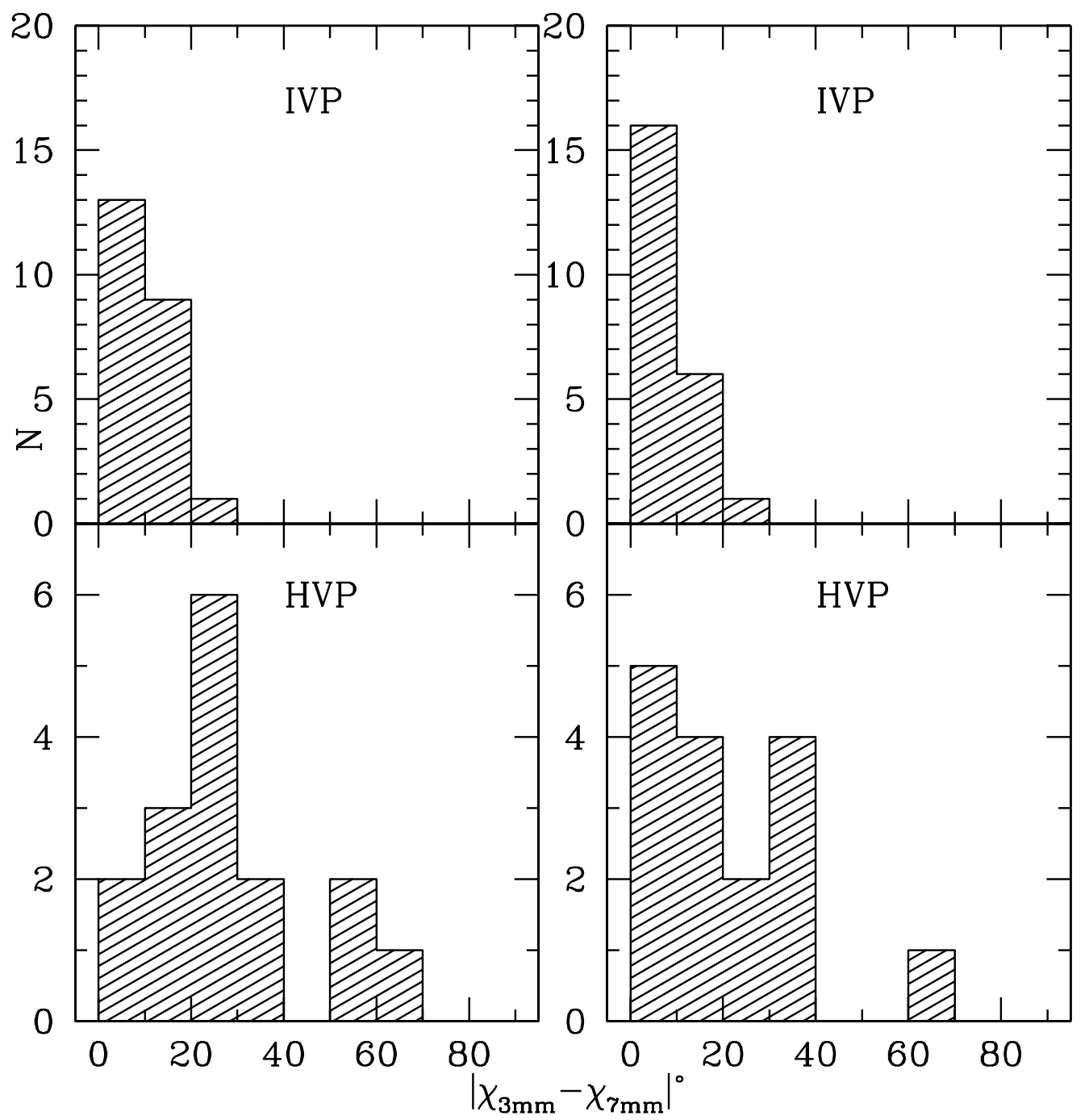

FIg. 8.-Distributions of offsets between EVPAs at $3 \mathrm{~mm}$ and in the $7 \mathrm{~mm}$ core before the RM correction (left) and after the RM correction (right). The distributions do not include the observations used to calculate the RM. 
quasars show only a moderate decrease of polarization from 1 to $7 \mathrm{~mm}$ at the epochs used to calculate RM $\left(m_{1 \mathrm{~mm}}=4.7 \% \pm\right.$ $1.6 \%$ and $m_{7 \mathrm{~mm}}=1.8 \% \pm 0.5 \%$ for $0528+134 ; m_{1 \mathrm{~mm}}=6.5 \% \pm$ $1.9 \%$ and $m_{7 \mathrm{~mm}}=4.0 \% \pm 0.7 \% \%$ for CTA 102$)$.

In a sample of 40 AGNs Zavala \& Taylor (2004) have determined RMs using VLBI images at $8-15 \mathrm{GHz}$ that are lower than we derive at millimeter wavelengths. The authors suggest that an external screen "in close proximity to the jet" is the most promising candidate for the source of the Faraday rotation. In this context, lower RMs obtained in the VLBI core at longer wavelengths might reflect a strong decrease in thickness of the screen with distance from the central engine. This is expected because the location of the core shifts with wavelength owing to optical depth effects. We assume a decreasing gradient in electron density of the screen, described by a power law, $N_{e} \propto d^{-a}$, where $d$ is the distance from the central engine. The RM depends on the density and magnetic field parallel to the line of sight, $B_{\|}$, integrated along the path through the screen to the observer: $\mathrm{RM} \propto \int N_{e} B \| d l$. In the absence of a velocity gradient across the jet, the magnetic field along the jet scales as $B_{z} \propto d^{-2}$, and the magnetic field transverse to the jet scales as $B_{\phi} \propto d^{-1}$ (Begelman et al. 1984). If the Faraday screen is at least mildly relativistic and has a helical field (Gabuzda 2006), $B_{\phi}$ provides the main contribution to the magnetic field component along the line of sight; therefore, $B_{\|} \propto d^{-1}$. This leads to $|\mathrm{RM}| \propto d^{-a}$ under the approximation that $l \propto d$. The location of the core from the central engine depends on the frequency of the observation $\nu$ as derived, for example, in Lobanov (1998): $d_{\text {core }, \nu}=\left(B_{1}^{k_{b}} F / \nu\right)^{1 / k_{r}}$, where $B_{1}$ is the magnetic field at distance $1 \mathrm{pc}$ from the central engine, $F$ is a function of the redshift and parameters depending on the jet geometry and relativistic electron energy distribution, and the equipartion value $k_{r}=1$. For a source with a flat millimeter-wave spectrum $d_{\text {core }, \nu} \propto \nu^{-1}$, which yields the dependence of the RM observed in the core on the frequency of observation as $\left|\mathrm{RM}_{\text {core }, \nu}\right| \propto \nu^{a}$. Therefore, values of RM obtained with different sets of frequencies should produce an estimate of the parameter $a$. We assume that the $\mathrm{RM}^{0}$ derived from polarization observations of the core at frequencies $\nu_{1}, \nu_{2}$, and $\nu_{3}$, where $\nu_{3}$ is the lowest one, yields the intrinsic RM at the location $d_{\text {core, } \nu_{3}}$.

Table 7 contains the published RMs obtained in the VLBI core at wavelengths longer than $7 \mathrm{~mm}, \mathrm{RM}_{15 \mathrm{GHz}}^{0}$ and $\mathrm{RM}_{8 \mathrm{GHz}}^{0}$, and derived values of $a$. For sources with $\mathrm{RM}^{0}$ available at more than two wavelengths, $a$ is calculated using a least-squares method. Table 7 does not show any difference in the values of $a$ between the HVP and IVP sources. The average $\langle a\rangle=1.8 \pm 0.5$ is similar to the value $a=2$ expected for outflow in a spherical or conical wind. This implies that an outflowing sheath wrapped around a conically expanding jet is a reasonable model for the foreground screen, consistent with the finding of Zavala \& Taylor (2004). The sheath can result from a mildly relativistic outer wind that emanates from the accretion disk and confines the inner relativistic jet (Gracia et al. 2005). According to the $\mathrm{RM}^{0}$ values in Table 7, the thickness of the sheath is higher in the HVP sources with respect to the IVP sources, a situation that should assist in stronger confinement of the jet of HVP blazars. The latter may help to explain the finding by J05 that the jet opening angles for quasars are smaller than for BL Lac objects.

We have corrected the values of $\chi_{3 \mathrm{~mm}}$ and $\chi_{7 \mathrm{~mm}}$ in the HVP and IVP sources for Faraday rotation by applying the corrections listed in Table 7 to the EVPAs given in Tables 3 and 4. The adjusted values of the EVPAs are used in the subsequent analysis.

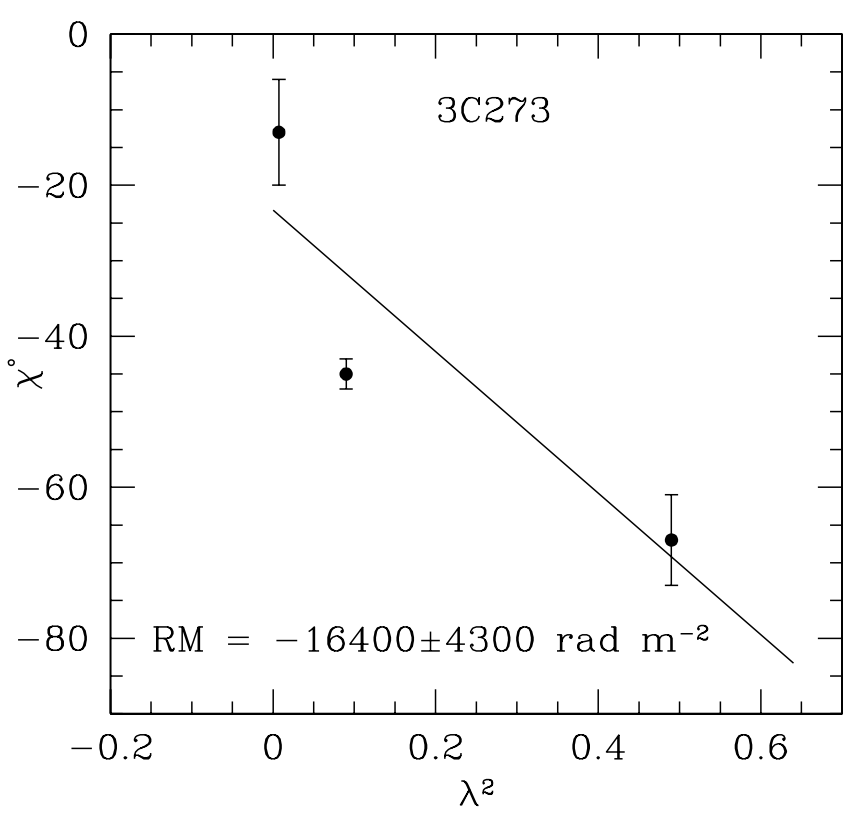

FIG. 9.-Dependence of polarization position angle on square of wavelength in $3 \mathrm{C} 273$. The solid line represents an approximation of the dependence by a $\lambda^{2}$ Faraday rotation law.

\subsection{Faraday Rotation in the LVP Sources}

In the LVP sources, the core at $7 \mathrm{~mm}$ is unpolarized; hence, the polarization position angle of the inner jet is defined by the polarization of VLBI components within a few milliarcseconds of the core. For the epochs shown in Figure 5 these are components $\mathrm{C} 1$ and $\mathrm{c} 1$ at $\sim 4$ mas in $3 \mathrm{C} 111$, components $\mathrm{t}$ and $\mathrm{u}$ at 1-2 mas in 3C 120, and component B2 at 1 mas in 3C 273. Figure 9 shows the $\lambda^{2}$ dependence of $\chi$ in $3 \mathrm{C} 273$, which yields a high $\mathrm{RM}, \mathrm{RM}=(1.6 \pm 0.5) \times 10^{4} \mathrm{rad} \mathrm{m}^{-2}$. The value is consistent with the high RM obtained for component Q6/W6 from the $43 / 86 \mathrm{GHz}$ images by Attridge et al. (2005), $\mathrm{RM}=2.1 \times$ $10^{4} \mathrm{rad} \mathrm{m}^{-2}$. However, a significant decrease in the density of the Faraday screen with distance from the core discussed above explains the good alignment between the EVPA at $1 \mathrm{~mm}$ and $\chi_{\text {jet }}$ when components are seen farther downstream. This occurs for 3C 111 and $3 \mathrm{C} 120$ : in $3 \mathrm{C} 111 \chi_{1 \mathrm{~mm}}=-54^{\circ} \pm 6^{\circ}$ and $\chi_{\text {jet }}=$ $-58^{\circ} \pm 10^{\circ}$, in $3 \mathrm{C} 120 \chi_{1 \mathrm{~mm}}=-38^{\circ} \pm 4^{\circ}$ and $\chi_{\text {jet }}=-35^{\circ} \pm 6^{\circ}$. The implication is that the magnetic fields in the $1 \mathrm{~mm}$ emission region have the same orientation as that in the jet features a few milliarcseconds from the core. Very low polarization in the VLBI core at $7 \mathrm{~mm}$, the high RM obtained close to the core in 3C 273, and consistency between the direction of polarization in the inner jet and at $1 \mathrm{~mm}$ in 3C 111 and 3C 120, all suggest that in the LVP sources the 1 and $3 \mathrm{~mm}$ cores have low polarization as well. The low polarization of the core could be the result of either finescale turbulence or depolarization by a very thick, inhomogeneous foreground screen with RM $>5 \times 10^{5} \mathrm{rad} \mathrm{m}^{-2}$, as suggested by Attridge et al. (2005).

The EVPAs of the LVP sources are not corrected for RM owing to the complexities discussed above. We use the simplification that, when strong polarized components are detected, they have already left the high-RM zone.

\section{CORRELATION ANALYSIS}

\subsection{Comparison of Polarization at Different Wavelengths}

We have calculated linear coefficients of correlation $f^{p}$ between the polarized flux densities in the core at $7 \mathrm{~mm}$ and overall 
TABLE 8

Correlation Coefficients between Polarization in the Core at $7 \mathrm{~mm}$ and Overall Polarization at Optical, $1 \mathrm{~mm}$, and $3 \mathrm{~mm}$ Wavelengths

\begin{tabular}{|c|c|c|c|c|c|c|}
\hline $\begin{array}{c}\text { Source } \\
\text { (1) }\end{array}$ & $\begin{array}{l}f_{o-7}^{p} \\
(2)\end{array}$ & $\begin{array}{l}f_{1-7}^{p} \\
(3)\end{array}$ & $\begin{array}{c}f_{3-7}^{p} \\
(4)\end{array}$ & $\begin{array}{c}r_{o-7}^{m} \\
(5)\end{array}$ & $\begin{array}{l}r_{1-7}^{m} \\
(6)\end{array}$ & $\begin{array}{l}r_{3-7}^{m} \\
(7)\end{array}$ \\
\hline 3C 66A............ & $0.81(4)^{*}$ & $0.22(8)$ & $0.81(3)$ & $0.97(4)^{*}$ & $0.21(8)$ & $0.81(3)$ \\
\hline $3 \mathrm{C} 111 \ldots \ldots \ldots \ldots$ & $\ldots(1)$ & $\ldots(0)$ & $\ldots(2)$ & $\ldots(0)$ & $\ldots(0)$ & $\ldots(0)$ \\
\hline $0420-014 \ldots \ldots \ldots \ldots \ldots \ldots$ & $0.67(4)$ & $0.94(5)^{*}$ & $\ldots(1)$ & $0.72(5)$ & $0.55(4)$ & $\ldots(1)$ \\
\hline 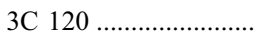 & $\ldots(1)$ & $\ldots(2)$ & $\ldots(1)$ & $\ldots(1)$ & $\ldots(2)$ & $\ldots(1)$ \\
\hline $0528+134 \ldots \ldots \ldots \ldots \ldots \ldots$ & $\ldots(1)$ & $-0.17(8)$ & $-0.38(3)$ & $\ldots(1)$ & $-0.49(8)$ & $0.14(3)$ \\
\hline 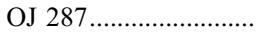 & $0.33(6)$ & $0.78(7)^{*}$ & $-0.11(5)$ & $0.30(6)$ & $0.68(7)^{*}$ & $0.25(5)$ \\
\hline 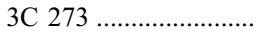 & $\ldots(1)$ & $\ldots(2)$ & $\ldots(2)$ & $\ldots(1)$ & $\ldots(2)$ & $\ldots(2)$ \\
\hline 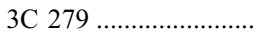 & $0.31(5)$ & $0.34(9)$ & $0.85(6)^{*}$ & $0.21(5)$ & $0.39(9)$ & $0.90(6)^{*}$ \\
\hline $1510-089 \ldots \ldots \ldots \ldots \ldots \ldots \ldots$ & $\ldots(1)$ & $0.79(5)$ & $0.97(4)^{*}$ & $0.08(3)$ & $0.65(5)$ & $0.92(4)^{*}$ \\
\hline 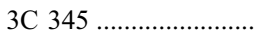 & $-0.19(4)$ & $0.30(6)$ & $0.59(5)$ & $-0.03(5)$ & $0.79(6)^{*}$ & $0.36(5)$ \\
\hline $1803+784 \ldots \ldots \ldots \ldots \ldots \ldots$ & $\ldots(0)$ & $-0.09(5)$ & $-0.09(5)$ & $\ldots(0)$ & $-0.70(5)$ & $0.61(5)$ \\
\hline $1823+568 \ldots \ldots \ldots \ldots \ldots \ldots$ & $\ldots(0)$ & $0.29(5)$ & $0.76(4)$ & $-0.89(3)$ & $0.30(6)$ & $0.94(4)^{*}$ \\
\hline BL Lac ......................... & $-0.97(3)^{*}$ & $-0.16(12)$ & $0.98(3)^{*}$ & $0.18(4)$ & $0.23(12)$ & $0.81(4)^{*}$ \\
\hline 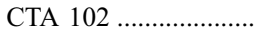 & $-0.68(3)$ & $0.34(11)$ & $0.90(4)^{*}$ & $-0.78(3)$ & $0.18(12)$ & $0.72(4)$ \\
\hline 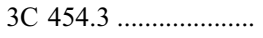 & $\ldots(2)$ & $-0.17(11)$ & $-0.14(3)$ & $-0.41(4)$ & $-0.14(11)$ & $-0.59(3)$ \\
\hline
\end{tabular}

NoтE.-Coefficients of correlation given with an asterisk are significant at a significance level $\epsilon=0.1$; integers in parentheses indicate number of observations.

polarized flux density measurements at (1) optical, $f_{o-7}^{p},(2) 1 \mathrm{~mm}$, $f_{1-7}^{p}$, and (3) $3 \mathrm{~mm}, f_{3-7}^{p}$. These apply to sources having data from at least three essentially simultaneous observations at two wavelengths. (Here we consider the observation at two wavelengths "simultaneous" if they are obtained within 2 weeks of each other.) We do the same for the correlation coefficients $r^{m}$ of the the degree of polarization in the core at $7 \mathrm{~mm}$ and the overall fractional polarization in each of the other wave bands, using the same subscript designations. For some sources several measurements have been carried out at high frequencies within 2 weeks of a few VLBA epochs. In these cases, we use only the observation that is nearest to the corresponding VLBA epoch. Table 8 contains coefficients of correlation $f_{o-7}^{p}, f_{1-7}^{p}$, and $f_{3-7}^{p}$ (cols. [2]-[4]) and $r_{0-7}^{m}, r_{1-7}^{m}$, and $r_{3-7}^{m}$ (cols. [5]-[7]). The number of points participating in the computation is indicated in parentheses. The coefficients of correlation that are significant at a level $\epsilon=0.1$ are indicated with asterisks.

The correlation coefficients between optical polarization and polarization in the core at $7 \mathrm{~mm}$ correspond to neither positive nor inverse correlation despite otherwise similar overall polarization characteristics (see $\S 3$ ). Blazars are known to have very short timescales of polarization variability at optical wavelengths ( $<1$ day, Hagen-Thorn 1980; Moore et al. 1987; Mead et al. 1990). Because of this, we suspect that the lack of exact simultaneity and the small number of observations affect the correlation analysis in the optical band.

The statistically significant coefficients of correlation listed in Table 8 indicate a strong positive correlation either between the polarized flux in the core at $7 \mathrm{~mm}$ and overall source at 1 and $3 \mathrm{~mm}$, or between the fractional polarization in the core and at shorter millimeter wavelengths. However, the number of sources with such behavior corresponds to $25 \%$ and $45 \%$ for the $1-7 \mathrm{~mm}$ and $3-7 \mathrm{~mm}$ data, respectively. The absence of statistically meaningful correlations for other sources might be explained by either small-number statistics, e.g., for $0420-014$ or $1510-089$, or by contributions from jet components different from the core to the polarized flux at 1 and $3 \mathrm{~mm}$. Table 9 gives the coefficients of correlation of the sources for which inclusion of the jet components (see $\S 4$ ) changes the significance of the correlation. Table 9 shows that including highly polarized components within a few milliarcseconds from the core dramatically improves the correlation in (1) all the LVP sources, (2) two of the HVP sources (CTA 102 and 3C 454.3), and (3) none of the IVP sources. For 3C 279 the correlation coefficient between the 7 and $1 \mathrm{~mm}$ polarization increases $\left(r_{1-7}^{m}=0.51\right)$ but does not change its significance substantially.

\subsection{Polarization-Total Flux Connection at Different Wavelengths}

We have computed coefficients of correlation between the degree of polarization and total flux density, $r_{\lambda}$ (listed in Table 10), and between the polarized and total flux densities, $f_{\lambda}$ (given in Table 11). The coefficients are calculated for each source that has three or more simultaneous measurements of the two quantities at wavelength $\lambda$ (in this case the observations are completely simultaneous). Since the JCMT observations were performed at 1.35 and $0.85 \mathrm{~mm}$, we have adjusted flux densities and fractional polarization at $0.85 \mathrm{~mm}$ to $1.35 \mathrm{~mm}$ using the spectral indices $\alpha_{\mathrm{mm}}$ and $\alpha_{\mathrm{mm}}^{p}$ provided in Table 6 . The data at $1 \mathrm{~mm}$ are supplemented by the measurements obtained by Nartallo et al. (1998) at $1.1 \mathrm{~mm}$ for sources common to both samples: $0420-014,0528+134$, OJ 287, 3C 273, 3C 279, 3C 345, 1823+568, BL Lac, and $3 \mathrm{C} 454.3$. Coefficients of correlation $r_{7} \mathrm{~mm}$ and $f_{7 \mathrm{~mm}}$ correspond to the VLBI core only.

TABLE 9

Correlation Coefficients between Polarization in the Inner Jet at $7 \mathrm{~mm}$ and Overall Polarization at $1 \mathrm{~mm}$ and $3 \mathrm{~mm}$

\begin{tabular}{|c|c|c|c|c|}
\hline $\begin{array}{l}\text { Source } \\
\text { (1) }\end{array}$ & $\begin{array}{l}f_{1-7}^{p \prime} \\
(2)\end{array}$ & $\begin{array}{c}f_{3-7}^{p \prime} \\
(3)\end{array}$ & $\begin{array}{l}r_{1-7}^{m \prime} \\
(4)\end{array}$ & $\begin{array}{c}r_{3-7}^{m \prime} \\
(5)\end{array}$ \\
\hline 3C $111 \ldots \ldots \ldots \ldots$ & $0.25(7)$ & $\ldots(2)$ & $0.64(7)^{*}$ & $\ldots(2)$ \\
\hline 3C $120 \ldots \ldots \ldots$ & $0.87(5)^{*}$ & $\ldots$ (1) & $0.78(6)^{*}$ & $\ldots(1)$ \\
\hline 3C $273 \ldots \ldots \ldots \ldots$ & $0.79(8)^{*}$ & $0.98(5)^{*}$ & $0.41(9)$ & $0.99(5)^{*}$ \\
\hline CTA $102 \ldots \ldots \ldots$ & $0.70(12)^{*}$ & $0.51(4)$ & $0.53(12)^{*}$ & $0.80(4)^{*}$ \\
\hline 3C $454.3 \ldots \ldots \ldots$ & $0.36(13)$ & $0.22(3)$ & $0.61(13)^{*}$ & $0.12(3)$ \\
\hline
\end{tabular}

NoTE.-Coefficients of correlation given with an asterisk are significant at a significance level $\epsilon=0.1$; integers in parentheses indicate number of observations. 
TABLE 10

Correlation Coefficients between Degree of Polarization and Total Flux Density

\begin{tabular}{|c|c|c|c|c|}
\hline $\begin{array}{l}\text { Source } \\
\text { (1) }\end{array}$ & $\begin{array}{l}r_{\text {opt }} \\
(2)\end{array}$ & $\begin{array}{l}r_{1 \mathrm{~mm}} \\
(3)\end{array}$ & $\begin{array}{c}r_{3 \mathrm{~mm}} \\
\text { (4) }\end{array}$ & $\begin{array}{c}r_{7 \mathrm{~mm}} \\
(5)\end{array}$ \\
\hline 3C $66 \mathrm{~A} \ldots \ldots \ldots \ldots$ & $0.08(6)$ & $-0.44(11)$ & $0.37(10)$ & $0.67(17)^{*}$ \\
\hline $3 \mathrm{C} 111 \ldots$ & ..(1) & $-0.51(10)$ & $0.06(3)$ & .. (0) \\
\hline $0420-014 \ldots \ldots$ & $0.89(5)^{*}$ & $-0.06(11)$ & $\ldots(1)$ & $0.43(17)^{*}$ \\
\hline 3C $120 \ldots \ldots \ldots . . . .$. & $\ldots(2)$ & $-0.48(8)$ & $\ldots(2)$ & $\ldots(2)$ \\
\hline $0528+134 \ldots \ldots \ldots$ & $\ldots(1)$ & $-0.33(11)$ & -0.89 (3) & $0.02(17)$ \\
\hline OJ $287 \ldots \ldots \ldots \ldots$ & $0.10(8)$ & $0.02(17)$ & $-0.36(13)$ & $-0.28(17)$ \\
\hline 3C $273 \ldots \ldots \ldots$ & $-0.01(6)$ & $0.20(20)$ & $0.32(20)$ & $-0.06(5)$ \\
\hline 3C $279 \ldots \ldots \ldots . . . . .$. & $-0.13(7)$ & $0.23(22)$ & $-0.13(18)$ & $0.13(17)$ \\
\hline $1510-089$.. & $\ldots(2)$ & $-0.62(5)$ & $0.74(9)^{*}$ & $-0.18(17)$ \\
\hline 3C $345 \ldots \ldots \ldots \ldots$ & $0.98(5)^{*}$ & $-0.41(16)$ & $0.34(22)$ & $-0.23(17)$ \\
\hline $1803+784 \ldots \ldots \ldots$ & $\ldots(0)$ & $0.11(6)$ & $-0.35(20)$ & $0.48(17)^{*}$ \\
\hline $1823+568 \ldots \ldots \ldots$ & $\ldots(0)$ & $0.00(8)$ & $0.43(20)^{*}$ & $0.38(17)$ \\
\hline BL Lac ................. & $-0.57(5)$ & $-0.51(19)^{*}$ & $-0.07(6)$ & $-0.12(17)$ \\
\hline CTA $102 \ldots \ldots \ldots$ & $-0.43(3)$ & $0.25(13)$ & $0.36(5)$ & $0.20(17)$ \\
\hline 3C $454.3 \ldots \ldots \ldots$ & $0.44(3)$ & $0.14(14)$ & $-0.19(5)$ & $0.67(17)^{*}$ \\
\hline
\end{tabular}

Note.-Coefficients of correlation given with an asterisk are significant at a significance level $\epsilon=0.1$; integers in parentheses indicate number of observations.

Table 10 reveals that, in general, the total flux density and percentage polarization are not well correlated. However, at $1 \mathrm{~mm}$ the one statistically significant coefficient (for BL Lac) indicates a decrease of fractional polarization when the source brightens, while at optical, $3 \mathrm{~mm}$, and $7 \mathrm{~mm}$ wavelengths the few correlation coefficients that are meaningful correspond to a positive correlation between the total flux density and degree of polarization. An increase of the total flux at millimeter wavelengths is usually connected with the emergence a new superluminal component from the VLBI core (Savolainen et al. 2002). The component might have a different direction of polarization than the core and partially cancel the observed polarization, thus leading to a poor correlation between the total flux and degree of polarization. Table 11, which lists correlation coefficients between the total and polarized flux densities, yields a qualitatively different result. Significant coefficients of correlation indicate that the polarized flux density increases as the total flux density rises at all wavelengths. The correlation occurs for $44 \%, 47 \%, 46 \%$, and $77 \%$ of sources at optical, $1 \mathrm{~mm}, 3 \mathrm{~mm}$, and $7 \mathrm{~mm}$ wavelengths, respectively.

Figure 10 shows the dependence between the percentage polarization and total flux density at $1 \mathrm{~mm}$ and in the core at $7 \mathrm{~mm}$ for HVP and IVP sources. For all 12 sources, the fractional polarization at maximum flux density in the VLBI core exceeds the average degree of polarization (dotted line) while at $1 \mathrm{~mm}$ eight sources show the inverse relation: the fractional polarization at maximum flux density is lower than the average. The exceptions are 3 C 279, CTA 102, and 3C 454.3, with all having jet components different from the core that appear to contribute to the emission at $1 \mathrm{~mm}$. The source $1803+784$ is another exception; it has the smallest range of flux variability at $1 \mathrm{~mm}$.

Despite poor correlation between the total flux density and percent polarization, which can be partially affected by complex polarization structure of the millimeter-wave core region during ejection of a new VLBI component, the data support the inference that the fractional polarization at $1 \mathrm{~mm}$ tends to decrease when a source brightens. In contrast, in the core at $7 \mathrm{~mm}$ the degree of polarization rises along with the total flux density. An increase of both the degree of polarization and polarized flux density as the VLBI core brightens implies that the flux increase is accompanied
TABLE 11

Correlation Coefficients between Polarized and Total Flux Density

\begin{tabular}{|c|c|c|c|c|}
\hline $\begin{array}{l}\text { Source } \\
\text { (1) }\end{array}$ & $\begin{array}{l}f_{\text {opt }} \\
(2)\end{array}$ & $\begin{array}{c}f_{1 \mathrm{~mm}} \\
(3)\end{array}$ & $\begin{array}{c}f_{3 \mathrm{~mm}} \\
(4)\end{array}$ & $\begin{array}{c}f_{7 \mathrm{~mm}} \\
(5)\end{array}$ \\
\hline 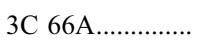 & $0.61(6)$ & $0.12(11)$ & $0.60(10)^{*}$ & $0.82(17)^{*}$ \\
\hline 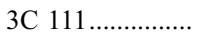 & $\ldots(1)$ & $0.24(10)$ & $0.15(3)$ & $\ldots(0)$ \\
\hline $0420-014 \ldots \ldots \ldots$ & $0.92(5)^{*}$ & $0.88(11)^{*}$ & $\ldots(1)$ & $0.84(17)^{*}$ \\
\hline 3C $120 \ldots \ldots \ldots \ldots$ & $\ldots(2)$ & $0.05(8)$ & $\ldots(2)$ & .. (2) \\
\hline $0528+134 \ldots \ldots \ldots$ & $\ldots(1)$ & $0.32(11)$ & $-0.81(3)$ & $0.55(17)^{*}$ \\
\hline 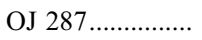 & $0.72(8)^{*}$ & $0.44(17)^{*}$ & $0.16(13)$ & $0.60(17)^{*}$ \\
\hline 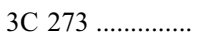 & $0.21(6)$ & $0.76(20)^{*}$ & $0.57(20)^{*}$ & $0.49(5)$ \\
\hline 3C $279 \ldots \ldots \ldots \ldots$ & $0.78(7)^{*}$ & $0.60(22)^{*}$ & $0.61(18)^{*}$ & $0.69(17)^{*}$ \\
\hline $1510-089 \ldots \ldots \ldots$ & $\ldots(2)$ & $0.03(5)$ & $0.81(9)^{*}$ & $0.06(17)$ \\
\hline 3C $345 \ldots \ldots \ldots \ldots$ & $0.99(5)^{*}$ & $0.21(16)$ & $0.74(22)^{*}$ & 0.39 (17) \\
\hline $1803+784 \ldots \ldots \ldots$ & $\ldots(0)$ & $0.26(6)$ & $-0.20(20)$ & $0.66(17)^{*}$ \\
\hline $1823+568 \ldots \ldots \ldots$ & $\ldots(0)$ & $0.77(8)^{*}$ & $0.72(20)^{*}$ & $0.79(17)^{*}$ \\
\hline 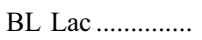 & $-0.14(5)$ & $0.30(19)$ & $0.67(6)$ & $0.66(17)^{*}$ \\
\hline CTA $102 \ldots \ldots \ldots . . .$. & $-0.30(3)$ & $0.60(13)^{*}$ & $0.52(5)$ & $0.76(17)^{*}$ \\
\hline 3C $454.3 \ldots \ldots \ldots . . .$. & $0.63(3)$ & $0.80(20)^{*}$ & $0.08(5)$ & $0.91(17)^{*}$ \\
\hline
\end{tabular}

NotE.-Coefficients of correlation given with an asterisk are significant at a significance level $\epsilon=0.1$; integers in parentheses indicate number of observations.

by ordering of the magnetic field in the emission region. The two opposing tendencies seen at $1 \mathrm{~mm}$, lower fractional polarization but higher polarized flux as the total flux becomes higher, indicate that the flux increases as emission with weaker (but nonzero) polarization becomes prominent.

\section{POLARIZATION POSITION ANGLE BEHAVIOR}

\subsection{Comparison of Polarization Position Angle at Different Wavelengths}

For the IVP and HVP sources we have computed values of deviation between optical and millimeter-wave EVPAs, $\Delta \chi_{\lambda_{1} \lambda_{2}}=$ $\left|\chi_{\lambda_{1}}-\chi_{\lambda_{2}}\right|$, where $\lambda_{1}$ is the optical wavelength and $\lambda_{2}$ is 1,3 , or $7 \mathrm{~mm}$, for measurements obtained within 2 weeks of each other (recall that $\chi_{7 \mathrm{~mm}}$ corresponds to the EVPA in the VLBI core). Figure 11 presents the distributions of $\Delta \chi_{\lambda_{1} \lambda_{2}}$.

Figure 11 shows that the IVP sources possess excellent agreement between polarization position angle at different wavelengths: $87 \%$ of $\Delta \chi_{o-1}(8), 92 \%$ of $\Delta \chi_{o-3}$ (12), and $90 \%$ of $\Delta \chi_{o-7}$ (21) fall into the range $0^{\circ}-20^{\circ}$ (the number in parentheses indicates the number of observations). The distribution of $\Delta \chi_{o-7}$ for the IVP sources agrees very well with the distribution of $\left|\chi_{\mathrm{opt}}-\chi_{\mathrm{core}, 7 \mathrm{~mm}}\right|$ obtained by Gabuzda et al. (2006) for $11 \mathrm{BL}$ Lac objects and 3C 279. This suggests that in the majority of BL Lac objects (1) alignment between the optical polarization position angle and EVPA in the VLBI core at $7 \mathrm{~mm}$ is a common feature, and (2) good agreement of the EVPA at different wavelengths from optical to millimeter wavelengths is expected.

For the HVP sources, the result is qualitatively different: $31 \%$ of $\Delta \chi_{o-1}$ (13), $55 \%$ of $\Delta \chi_{o-3}$ (11), and $64 \%$ of $\Delta \chi_{o-7}$ (22) are located within $20^{\circ}$. The best agreement is observed between optical EVPA and polarization position angle in the core at $7 \mathrm{~mm}$, and between optical and $3 \mathrm{~mm}$ EVPA, while the distribution of $\Delta \chi_{o-1}$ indicates significantly larger misalignments between polarization position angles.

In the LVP sources the EVPA at $7 \mathrm{~mm}$ corresponds to $\chi_{\text {jet }}$, defined by jet components within a few milliarcseconds of the core. Since the EVPAs are not corrected for RM, distributions of $\Delta \chi_{\lambda_{1} \lambda_{2}}$ are constructed for all possible pairs of wavelengths (Fig. 12). Figure 12 shows that for the LVP sources the best agreement in direction of polarization occurs between 1 and 


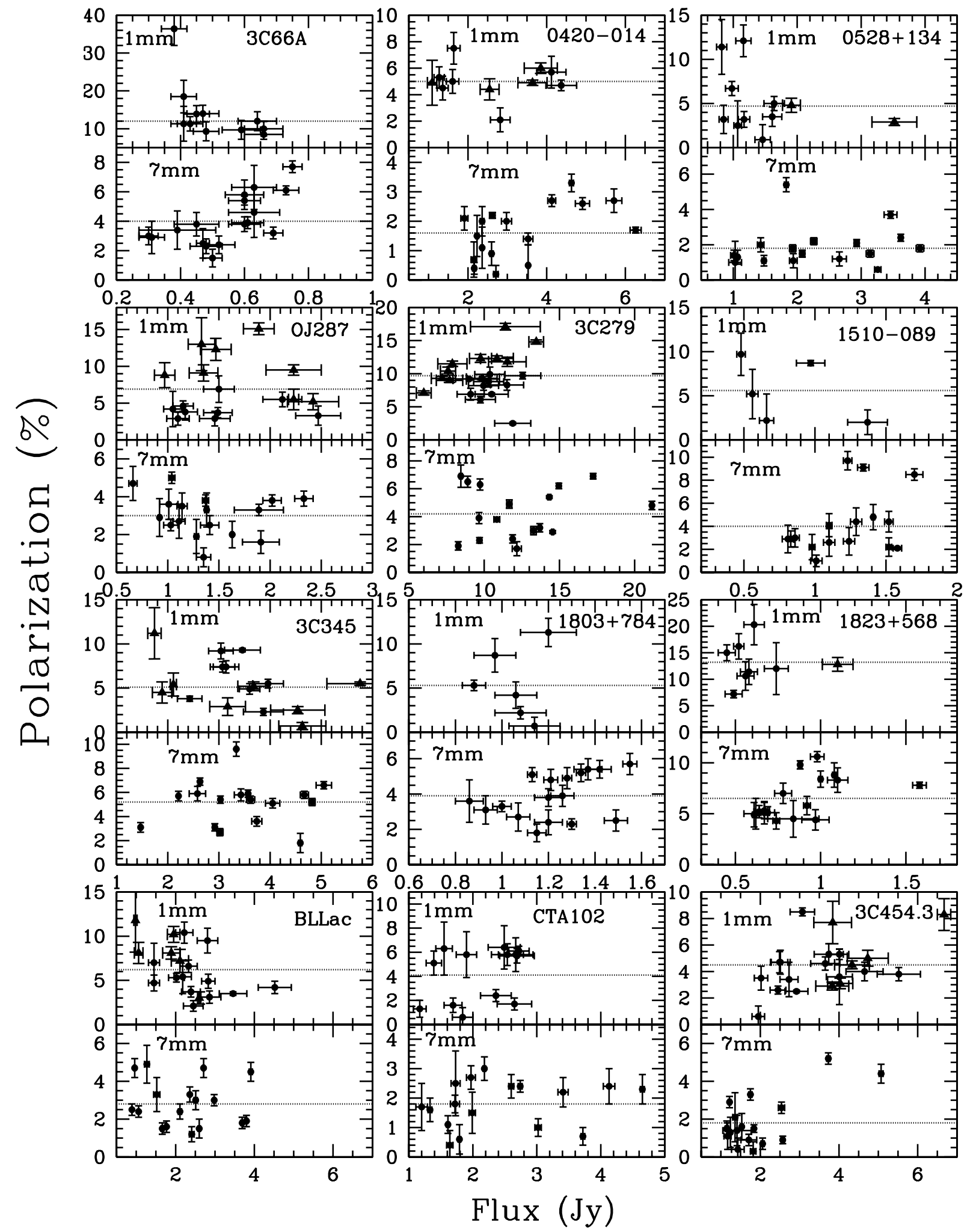

Fig. 10.-Fractional polarization vs. total flux density at $1 \mathrm{~mm}$ from the whole source and in the core at $7 \mathrm{~mm}$. Filled circles correspond to our observations, and triangles repesent the data at $1.1 \mathrm{~mm}$ from Nartallo et al. (1998).

$7 \mathrm{~mm}$, as well as between 3 and $7 \mathrm{~mm}$. This result, plus a good correlation between the fractional polarization light curves at these wavelengths found in $\S 6.1$, imply that in the LVP sources (1) the millimeter-wave core has weak polarization and (2) high polarization at the short millimeter wavelengths $(>2 \%-3 \%)$ originates in a jet component within a few milliarcseconds of the core.
The findings require either (1) negligible Faraday depolarization at $7 \mathrm{~mm}$ and intrinsically low polarization in the core at submillimeter wavelengths, or (2) a highly Faraday-thick screen near the core region, causing depolarization of the core at $1 \mathrm{~mm}$. The latter is a more suitable explanation due to the high Faraday rotation in the region nearest to the core, as discussed in $\S 5.2$. 


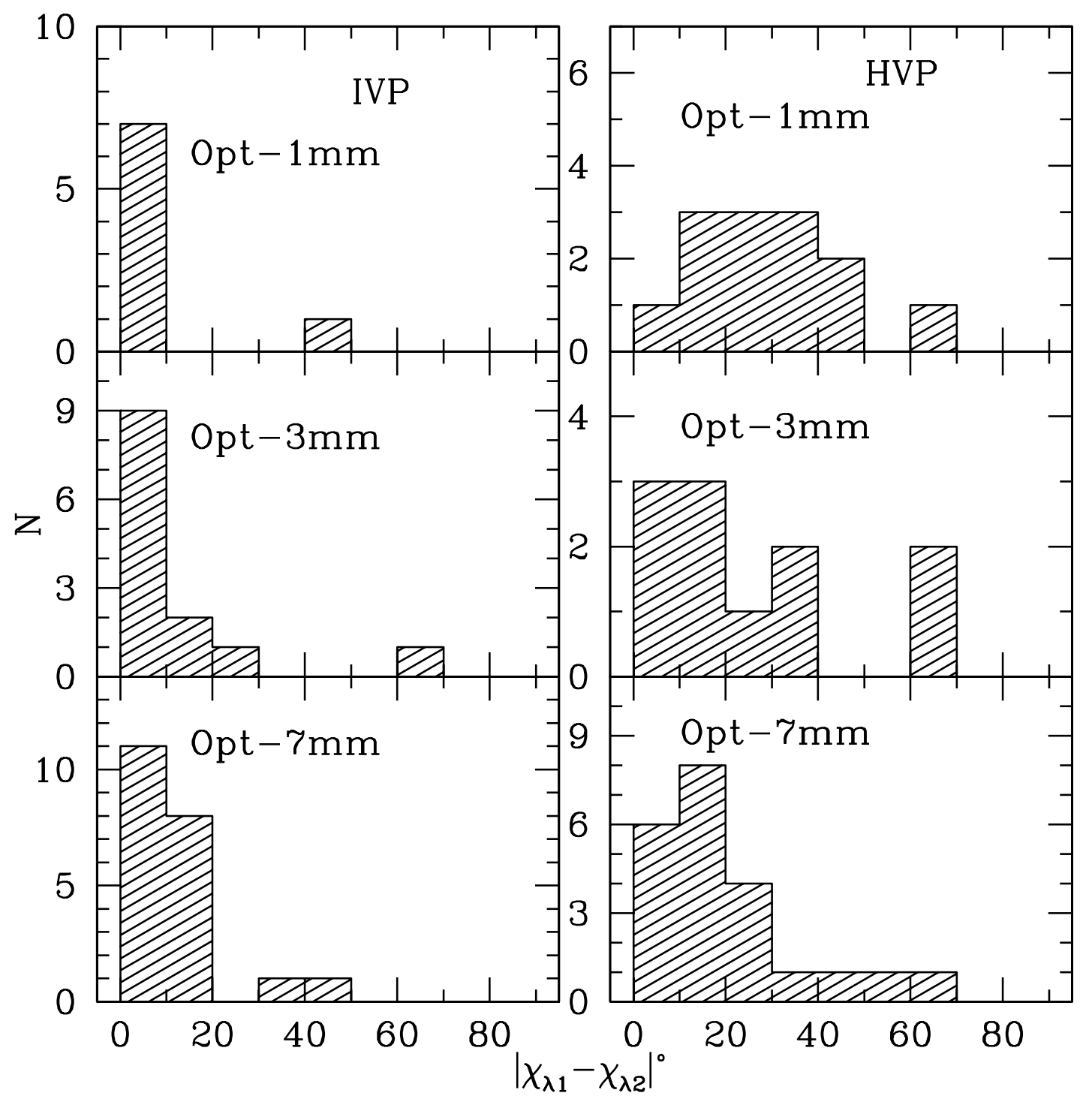

FIg. 11.-Distributions of offsets between EVPAs for simultaneous observations at different wavelengths in the IVP and HVP sources.

Comparison of EVPAs obtained at different wavelengths indicates that in the highly optically polarized sources (the IVP and HVP blazars) the optical polarization position angle tends to align with the EVPA in the VLBI core, while the polarization position angle at $1 \mathrm{~mm}$ in the HVP sources shows the weakest connection with the optical polarization position angle.

\subsection{Direction of Polarization Relative to the Jet Axis}

We have compared the polarization position angle, $\chi_{\lambda}$, for each polarization measurement with the position angle of the jet projected on the sky, $\Theta_{\text {jet }}$ (Table 4); $\Theta_{\text {jet }}$ corresponds to the nearest VLBA epoch to a given polarization measurement. Figure 13 gives the distributions of the offsets between direction of polarization and jet axis, $\left|\chi_{\lambda}-\Theta_{\text {jet }}\right|$, for polarization measured at the different wavelengths, $\lambda$, and for the different groups of objects. In the case of the IVP and HVP sources, $\chi_{7 \mathrm{~mm}}$ corresponds to the EVPA in the core; in the LVP group $\chi_{7 \mathrm{~mm}}$ is equal to $\chi_{\text {jet }}$ as defined in $\S 4$. The EVPAs at 3 and $7 \mathrm{~mm}$ for the HVP and IVP sources are corrected for RM, and the distributions at these wavelengths should be similar to that at $1 \mathrm{~mm}$ if the RM variability is not significant. It is possible though that the difference in the number of observations at the millimeter wavelengths can introduce some discrepancy between the distributions.

Figure 13 shows obvious alignment of the electric vector with the jet direction in the IVP sources: $75 \%$ of $\chi_{\text {opt }}(36), 59 \%$ of $\chi_{1 \mathrm{~mm}}$ (56), $76 \%$ of $\chi_{3 \mathrm{~mm}}$ (29), and $64 \%$ of $\chi_{7 \mathrm{~mm}}$ (102) lie within $20^{\circ}$ of the inner jet direction (the numbers in parentheses indicate the number of observations at each wavelength), although the widest range of offsets is observed at $1 \mathrm{~mm}$. In contrast, the HVP sources do not show any significant relation between EVPA and jet direction, and the values of $\chi_{\lambda}$ are distributed almost uniformly from $0^{\circ}$ to $90^{\circ}$ with respect to the jet direction at all wavelengths. An exception is the distribution at $1 \mathrm{~mm}$, which contains two peaks at intervals $0^{\circ}-20^{\circ}$ and $80^{\circ}-90^{\circ}$, suggesting that there is a preference for the electric vector to lie along or perpendicular to the jet. The difference between the distributions of the IVP and HVP sources is similar to the difference between the distributions of core electric vector offsets from the jet direction found by Lister \& Homan (2005) for BL Lac objects and quasars, respectively, in the Monitoring of Jets in AGNs with VLBA Experiments (MOJAVE) survey at $2 \mathrm{~cm}$. Although our dichotomy is not based simply on the optical classification of an object, we conclude that this difference persists from centimeter to optical wavelengths and implies 


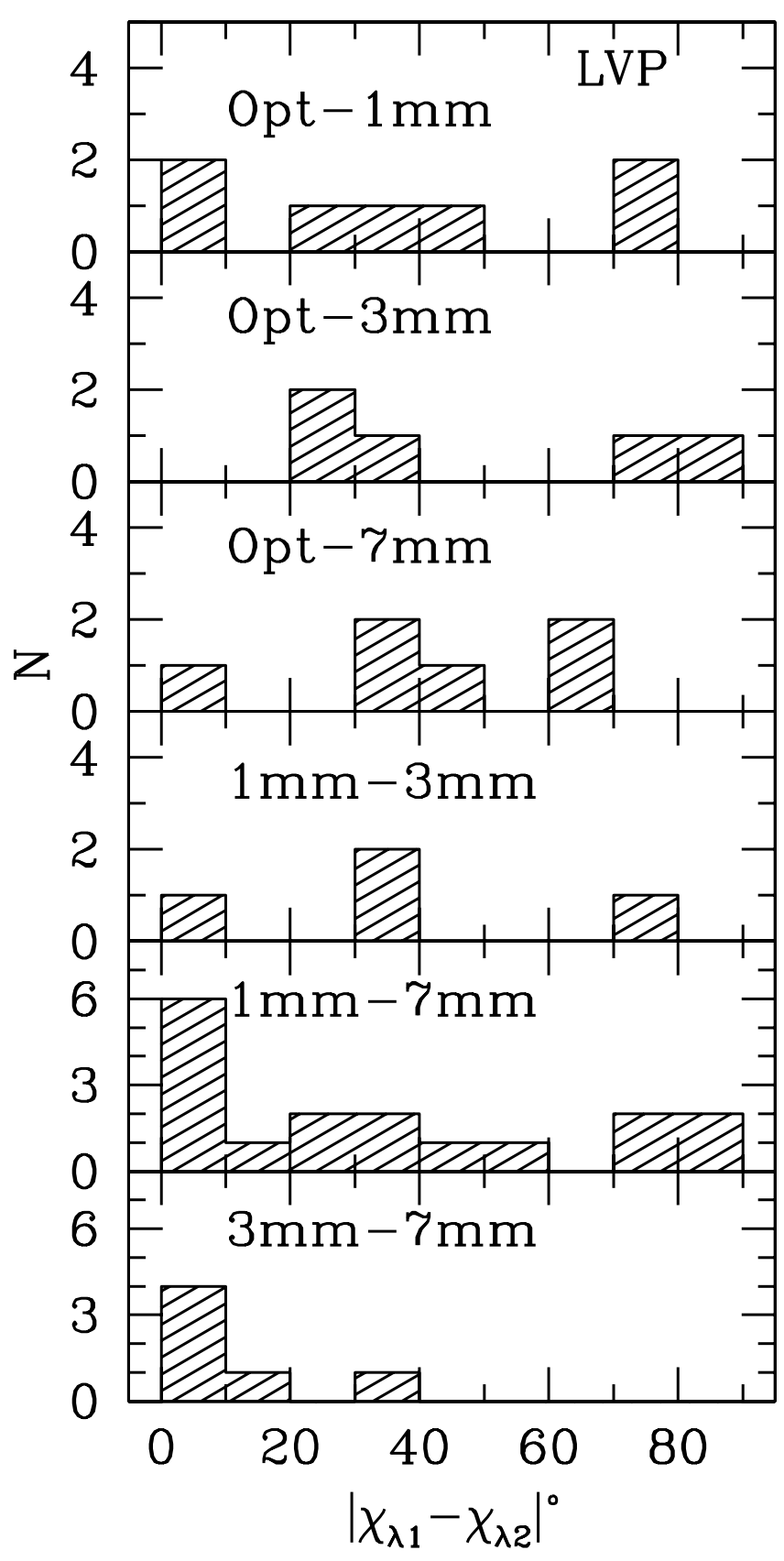

FIG. 12.-Distributions of offsets between EVPAs for simultaneous observations at different wavelengths in the LVP sources. The polarization position angle at $7 \mathrm{~mm}$ corresponds to the EVPA in the inner jet.

differences in the magnetic fields and/or processes responsible for the polarized emission in these objects.

In the LVP sources the distributions of $\left|\chi_{\lambda}-\Theta_{\text {jet }}\right|$ at optical and $1 \mathrm{~mm}$ wavelengths do not support a connection of the EVPA with the jet direction, although the polarization at $1 \mathrm{~mm}$ seems to avoid being parallel to the jet. The polarization position angle at $3 \mathrm{~mm}$ and in the inner jet at $7 \mathrm{~mm}$ clearly reveals a preferential direction perpendicular to the jet: $89 \%$ of $\chi_{3} \mathrm{~mm}(9)$ and $62 \%$ of $\chi_{7 \mathrm{~mm}}$ (47) values lie within $70^{\circ}-90^{\circ}$ of the jet axis. Although the distribution at $3 \mathrm{~mm}$ is dominated by the observations of $3 \mathrm{C} 273$, all three sources make a significant contribution in the pronounced peak of the distribution at $7 \mathrm{~mm}$.

The properties of polarization position angle with respect to the jet direction are distinct for each group: good alignment of the electric vector with the jet direction at all wavelengths in the
IVP sources, chaotic behavior of the electric vector in the HVP blazars independent of frequency, and electric vector preferentially transverse to the jet direction in the LVP objects at $3 \mathrm{~mm}$ and in the inner jet at $7 \mathrm{~mm}$. It is noteworthy that the dichotomy at high frequencies is similar to that found for regions farther out in the jet at lower frequencies (Cawthorne et al. 1993; Lister \& Homan 2005).

\section{INTERPRETATION}

\subsection{Magnetic Turbulence}

Our data are consistent with the assumption that we observe incoherent synchrotron radiation across the wavelength range from optical to millimeter wavelengths. A uniform magnetic field throughout the emission region yields the maximum possible degree of polarization, $m_{\max }=(\alpha+1) /(\alpha+5 / 3)$ (e.g., Pacholczyk 1970), which is $\sim 70 \%$ at millimeter wavelengths ( $\alpha \sim 0.5$; Table 6$)$ and $\sim 78 \%$ in the optical band $(\alpha \sim 1.4$; Impey \& Neugebauer 1988). Such a high degree of polarization has never been observed in a blazar. The polarization can be significantly reduced if the emission region is turbulent enough that it is composed of many cells, each containing a roughly uniform magnetic field that is randomly oriented relative to that in other cells (Burn 1966; Burch 1979). For $N$ cells within a telescope beam, the fractional polarization is given by the equation (Hughes \& Miller 1991)

$$
m \approx m_{\max } / \sqrt{N} \pm m_{\max } / \sqrt{2 N}
$$

The results obtained in $\S \S 6-7$ indicate that the polarization properties, and therefore magnetic field structure, of our sources are comparable within a group but diverse across different groups in our classification scheme. We have calculated for each group the mean and standard deviation of the maximum degree of polarization over sources measured at each wavelength, $\left\langle m_{\lambda}^{\max }\right\rangle \pm$ $\sigma_{\lambda}^{\max }$, as well as similar values, $\left\langle m_{\lambda}^{\min }\right\rangle \pm \sigma_{\lambda}^{\min }$, for the minimum degree of polarization. The values, given in Table 12, show that both the maximum and minimum degree of polarization increase with frequency except for the LVP group, within which the optical polarization is similar to the polarization in the VLBI core. At a given frequency, the fractional polarization is lowest for the LVP objects and highest for the IVP sources, independent of whether the polarization is near the minimum or maximum. We use the means and standard deviations given in Table 12 and equation (3) to determine whether the observed parameters can be derived within a cellular model. Table 12 lists the number of cells, $N_{\text {cell }}^{\max }$ and $N_{\text {cell }}^{\text {min }}$, that result in the observed degree of polarization under this model.

We can calculate the number of turbulent cells for any degree of polarization; however, the same number should account for the standard deviation as well. We find that this requirement is not satisfied at high-polarization states, where the standard deviation is significantly lower than the model predicts. In lowpolarization states, the requirement holds at all wavelengths except $1 \mathrm{~mm}$, where the standard deviations for the LVP and HVP sources are slightly lower than expected according to the derived number of cells. This suggests that the high-polarization states involve an additional process that provides some order to the magnetic field, while the low-polarization states can be explained within the cellular model.

Three interesting consequences follow from analysis of Table 12. First, the observed difference in degree of polarization among the groups of objects suggests that the emission regions of the LVP and HVP sources contain more turbulent cells than 


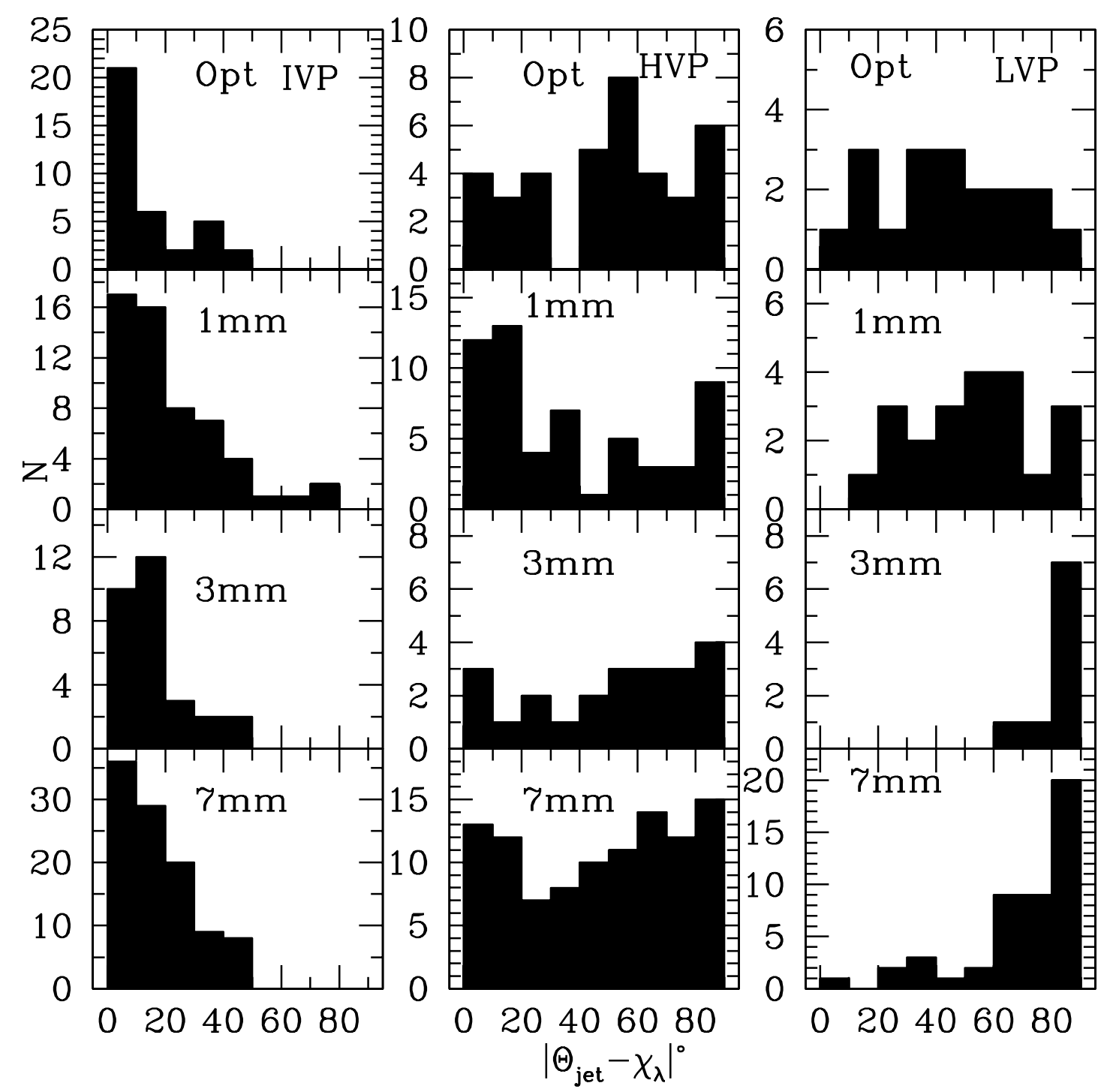

FIG. 13.-Distributions of offsets between polarization position angle and jet direction.

TABLE 12

Average Values of Fractional Polarization

\begin{tabular}{|c|c|c|c|c|c|}
\hline Group & Quantity & Opt & $1 \mathrm{~mm}$ & $3 \mathrm{~mm}$ & $7 \mathrm{~mm}$ \\
\hline \multirow[t]{2}{*}{ 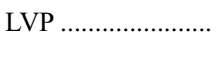 } & $\left\langle m_{\max }\right\rangle(\%)$ & $1.4 \pm 1.2(1.0)$ & $10.2 \pm 2.1(7.2)$ & $2.8 \pm 0.9(2.0)$ & $1.4 \pm 0.3(1.0)$ \\
\hline & $N_{\text {cell }}^{\max }$ & 3104 & 47 & 625 & 2500 \\
\hline \multirow[t]{2}{*}{ HVP.................... } & $\left\langle m_{\max }\right\rangle(\%)$ & $11.4 \pm 7.5(8.1)$ & $8.6 \pm 1.8(6.1)$ & $3.1 \pm 1.3(2.2)$ & $6.0 \pm 1.9(4.2)$ \\
\hline & $N_{\text {cell }}^{\max }$ & 47 & 66 & 510 & 136 \\
\hline \multirow[t]{2}{*}{ IVP } & $\left\langle m_{\max }\right\rangle(\%)$ & $29.0 \pm 11.3(20)$ & $16.8 \pm 9.4(11)$ & $9.1 \pm 2.4(6.4)$ & $7.5 \pm 2.1(5.3)$ \\
\hline & $N_{\text {cell }}^{\max }$ & 8 & 18 & 59 & 87 \\
\hline \multirow[t]{2}{*}{ LVP .................... } & $\left\langle m_{\min }\right\rangle(\%)$ & $0.4 \pm 0.3(0.3)$ & $1.3 \pm 0.3(0.9)$ & $0.9 \pm 0.6(0.6)$ & $0.1 \pm 0.1(0.1)$ \\
\hline & $N_{\text {cell }}^{\min }$ & 38025 & 2900 & 6050 & 490000 \\
\hline \multirow[t]{2}{*}{ HVP........................ } & $\left\langle m_{\min }\right\rangle(\%)$ & $1.7 \pm 1.2(1.2)$ & $1.6 \pm 0.8$ & $0.6 \pm 0.3(0.4)$ & $0.5 \pm 0.2(0.3)$ \\
\hline & $N_{\text {cell }}^{\min }$ & 2105 & 1914 & 13611 & 19600 \\
\hline \multirow[t]{2}{*}{ IVP ....................... } & $\left\langle m_{\min }\right\rangle(\%)$ & $10.6 \pm 8.2(7.5)$ & $4.0 \pm 3.1(2.8)$ & $3.2 \pm 2.1(2.3)$ & $2.0 \pm 1.0(1.4)$ \\
\hline & $N_{\text {cell }}^{\min }$ & 54 & 306 & 480 & 1225 \\
\hline
\end{tabular}

NoтE.-Values in parentheses indicate standard deviations expected from the cellular model with number of cells derived from the fractional polarization; see text. 


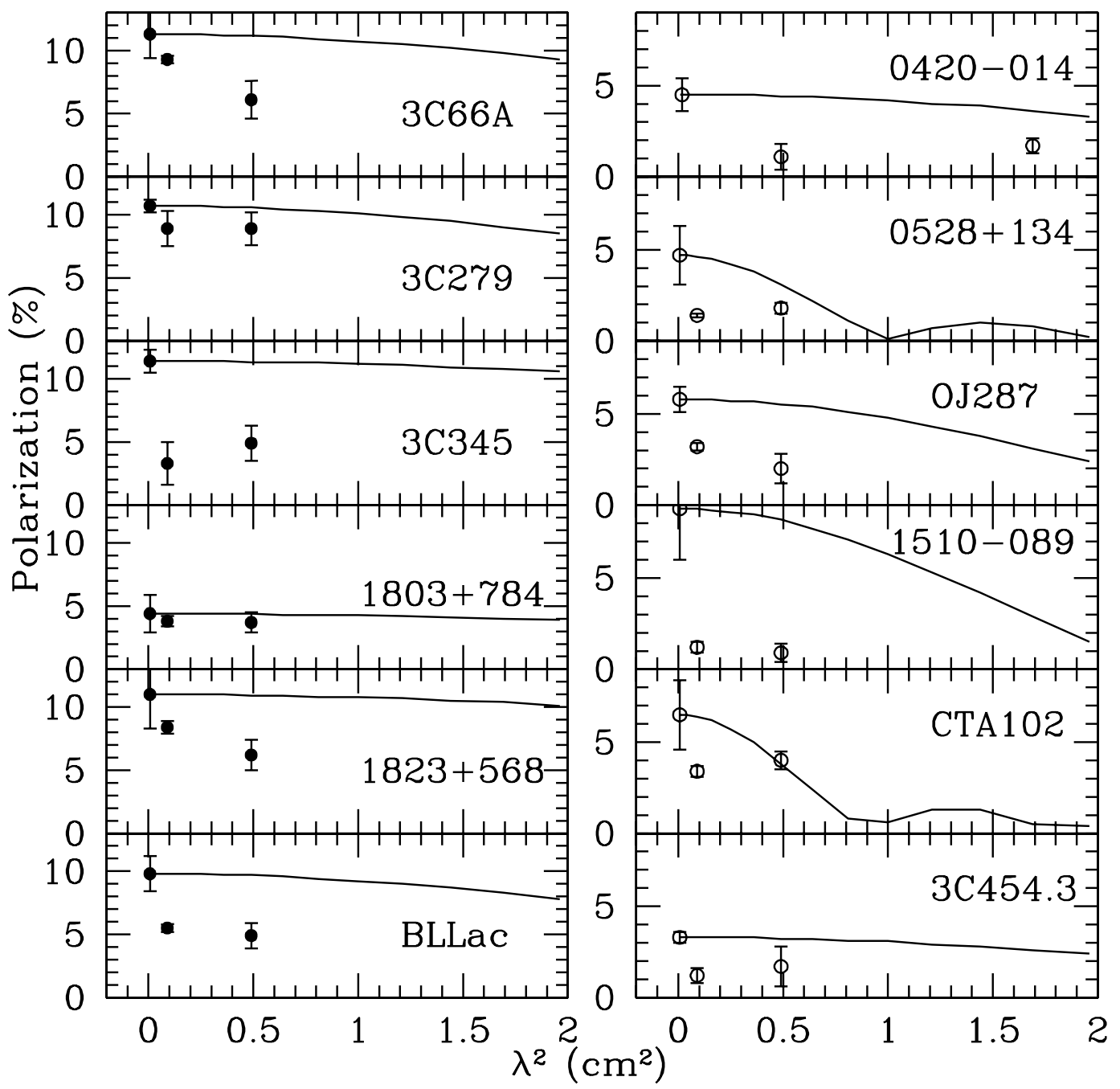

FIG. 14.-Dependence of degree of polarization on wavelength in the IVP (left) and HVP (right) sources. Solid lines represent the expected Faraday depolarization according to eq. (4) and RM derived in $\S$ 5.1. In general, the fit is poor, so that in most of our sources Faraday depolarization is unable to explain the frequency dependence of the polarization.

is the case for IVP objects; either the cells are finer in the LVP and HVP sources, or the emission region is smaller in the IVP blazars. Second, the fact that sources within a group have similar fractional polarization at different wavelengths implies that the emitting regions partially overlap. Third, the fact that fractional polarization decreases with wavelength suggests that the emitting region is larger at longer wavelengths.

Faraday rotation by a foreground screen can produce depolarization across a beam that also results in frequency dependence of the polarization (Burn 1966). However, Faraday depolarization cannot account for the frequency dependence among the groups at optical and $1 \mathrm{~mm}$ wavelengths, since the effect is negligible at such short wavelengths. We assume that turbulence and difference in volume of emission is responsible for the frequency dependence at optical and $1 \mathrm{~mm}$ wavelengths, but the decrease of polarization at longer millimeter wavelengths could be caused by Faraday depolarization. To calculate the frequency dependence of the polarization we apply a model proposed by Burn (1966) with the simplifications suggested by Zavala \& Taylor (2004),

$$
m(\%)=m_{\mathrm{RM}=0}\left|\operatorname{sinc}\left(\mathrm{RM} \lambda^{2}\right)\right|,
$$

where RM values are RMs derived in $\S 5.1$. We compare the model with the polarization at different wavelengths using the simultaneous data employed for RM estimates. In this case $m_{\mathrm{RM}=0}$ corresponds to $m_{1 \mathrm{~mm}}$ and is different for each source. Figure 14 shows the frequency dependence of the polarization according to the observations and equation (4). Figure 14 demonstrates that although for some sources (3C 279 and 1803+784) the dependence of the polarization on frequency can be caused by Faraday depolarization, for the majority of the objects the fractional polarization decreases with wavelength much faster than equation (4) predicts. It appears that depolarization from a gradient across the beam in a Faraday screen alone is unable to explain the observed frequency dependence of the polarization. A difference in the size of the emitting regions and/or in turbulence scale of the magnetic field is needed to account for the dependence.

\subsection{Shock Model versus the Observed Polarization}

A commonly occurring structure that leads to ordering of magnetic fields is a shock, which compresses the component of the magnetic field that lies parallel to the shock front. The significant dichotomy in degree of polarization at high-polarization states among the groups might then be explained by differences in shock strengths. Lister \& Smith (2000) proposed a similar explanation to account for differences in polarization properties of low and high optically polarized radio-loud quasars (LPRQs and HPQs, respectively), suggesting that LPRQs have weaker shocks than do 
TABLE 13

Derived Shock Strength

\begin{tabular}{|c|c|c|c|c|c|c|c|c|c|}
\hline $\begin{array}{c}\text { Source } \\
\text { (1) }\end{array}$ & $\begin{array}{c}m_{\mathrm{opt}}^{\max } \\
(\%) \\
(2)\end{array}$ & $\begin{array}{c}\eta_{\mathrm{opt}} \\
(3)\end{array}$ & $\begin{array}{c}m_{1 \mathrm{~mm}}^{\max } \\
(\%) \\
(4)\end{array}$ & $\begin{array}{c}\eta_{1 \mathrm{~mm}} \\
\quad(5)\end{array}$ & $\begin{array}{c}m_{3 \mathrm{~mm}}^{\max } \\
(\%) \\
(6)\end{array}$ & $\begin{array}{c}\eta_{3 \mathrm{~mm}} \\
(7)\end{array}$ & $\begin{array}{c}m_{7 \mathrm{~mm}}^{\max } \\
(\%) \\
(8)\end{array}$ & $\begin{array}{c}\eta_{7 \mathrm{~mm}} \\
(9)\end{array}$ & $\begin{array}{c}\Theta^{\prime} \\
(\mathrm{deg}) \\
(10)\end{array}$ \\
\hline 3C $66 \mathrm{~A} \ldots \ldots$ & 29.7 & 1.94 & 36.4 & 3.20 & 9.3 & 1.21 & 7.7 & 1.17 & 116.0 \\
\hline 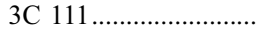 & 3.1 & 1.05 & 12.2 & 1.22 & 2.3 & 1.04 & 0.5 & 1.01 & 108.3 \\
\hline $0420-014 \ldots \ldots \ldots \ldots \ldots \ldots$ & 26.2 & 1.81 & 7.5 & 1.18 & 0.5 & 1.01 & 3.3 & 1.07 & 59.8 \\
\hline 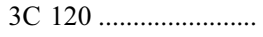 & 0.5 & 1.01 & 11.1 & 1.30 & 2.1 & 1.05 & 1.1 & 1.02 & 124.5 \\
\hline 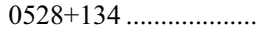 & 7.0 & 1.15 & 12.1 & 1.32 & 1.4 & 1.03 & 5.4 & 1.12 & 57.0 \\
\hline 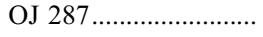 & 15.6 & 1.24 & 6.9 & 1.11 & 4.0 & 1.06 & 5.0 & 1.08 & 85.3 \\
\hline 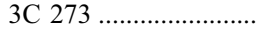 & 0.5 & 1.01 & 7.2 & 1.11 & 4.0 & 1.06 & 1.6 & 1.02 & 96.8 \\
\hline 3C $279 \ldots \ldots \ldots \ldots \ldots \ldots \ldots \ldots \ldots \ldots \ldots \ldots$ & 39.2 & 3.49 & 10.9 & 1.30 & 13.7 & 1.40 & 6.9 & 1.17 & 59.2 \\
\hline $1510-089$................... & 4.1 & 1.06 & 9.7 & 1.15 & 4.8 & 1.07 & 9.7 & 1.15 & 83.6 \\
\hline 3C $345 \ldots \ldots \ldots \ldots \ldots \ldots \ldots \ldots$ & 38.3 & 1.78 & 11.4 & 1.18 & 8.1 & 1.13 & 9.6 & 1.15 & 82.7 \\
\hline $1803+784 \ldots \ldots \ldots \ldots \ldots \ldots$ & $\ldots$ & $\ldots$ & 11.3 & 1.24 & 5.9 & 1.11 & 5.7 & 1.11 & 67.1 \\
\hline $1823+568 \ldots \ldots \ldots \ldots \ldots \ldots$ & 29.8 & $\ldots$ & 20.3 & $\ldots$ & 9.9 & 1.91 & 10.6 & 2.08 & 37.0 \\
\hline BL Lac ........................ & 7.8 & 1.12 & 10.4 & 1.18 & 7.8 & 1.13 & 4.9 & 1.08 & 86.3 \\
\hline CTA $102 \ldots \ldots \ldots \ldots \ldots \ldots$ & 9.4 & 1.14 & 7.1 & 1.12 & 3.4 & 1.06 & 6.1 & 1.10 & 75.9 \\
\hline 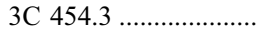 & 6.2 & 1.28 & 8.5 & 1.51 & 1.8 & 1.07 & 6.6 & 1.35 & 39.0 \\
\hline
\end{tabular}

HPQs. We use the Hughes \& Miller (1991) approach to estimate the shock strength for the sources in our sample,

$$
m \approx \frac{\alpha+1}{\alpha+5 / 3} \frac{\left(1-\eta^{-2}\right) \sin ^{2} \Theta^{\prime}}{2-\left(1-\eta^{-2}\right) \sin ^{2} \Theta^{\prime}},
$$

where $\eta=n_{\text {shocked }} / n_{\text {unshocked }}$ and $n$ is the number density of the plasma; $\Theta^{\prime}$ is the viewing angle of the jet corrected for relativistic aberration: $\Theta^{\prime}=\tan ^{-1}\left\{\sin \Theta_{o} / \Gamma\left[\cos \Theta_{o}-\left(1-\Gamma^{-2}\right)^{1 / 2}\right]\right\}$, where $\Gamma$ is the bulk Lorentz factor and $\Theta_{o}$ is the viewing angle of the jet in the observer's frame. The use of the viewing angle with respect to the jet axis in the formula applies to a transverse plane-wave shock, and the formula is valid for a shock propagating through a plasma with a completely chaotic magnetic field. The maximum possible polarization should be seen when the shock is viewed along the plane of compression in the frame of the shock (i.e., $\Theta^{\prime} \approx 90^{\circ}$ ). Columns (3), (5), (7), and (9) of Table 13 list the values of the estimated shock strength $\eta$ based on the maximum observed degree of polarization (cols. [2], [4], [6], and [8]) at each wavelength. Column (10) contains the viewing angle $\Theta^{\prime}$ in the shock frame, as derived from the values of $\left\langle\Theta_{o}\right\rangle$ and $\langle\Gamma\rangle$ obtained for each object in J05. Because we do not have multicolor data in the optical region, the computation of $\eta$ adopts $\alpha_{\mathrm{opt} / 1 \mathrm{~mm}}$ for the value of $\alpha_{\mathrm{opt}}$, while at 1,3 , and $7 \mathrm{~mm}$ we use the three-point spectral index $\alpha_{\mathrm{mm}}$ derived from all three millimeter wavelengths (see Table 6).

The shock strengths listed in Table 13 are similar among the sources and rather low, $\eta \sim 1.1-1.2$, and hence cannot be the primary reason behind the diversity in degree of polarization. Instead, we can explain this by differences in viewing angle relative to the plane of compression, although a very high level of polarization $(m \gtrsim 30 \%)$ does require much stronger compression, $\eta \sim 2-3$. Note that for 3C 66A J05 found a significant range of apparent speeds of superluminal components, from $1.5 c$ to $27 c$. We use the Lorentz factor and viewing angle that corresponds to the average of $\Gamma$ and $\Theta_{o}$ derived for the fast components only $\left(\Gamma=27.8\right.$ and $\left.\Theta_{o}=3.3^{\circ}\right)$, because $\langle\Gamma\rangle$ and $\left\langle\Theta_{o}\right\rangle$ averaged over all components (J05) fails to produce polarization as large as that observed for any given value of $\eta$. The latter is the case for $1823+568$ as well. However, for $1823+568$ moving components within 1 mas of the core were not observed (J05) and the jet parameters are obtained using kinematics of two components at $\sim 2$ mas from the core. This implies that $\Gamma$ and $\Theta_{o}$ in the VLBI core of $1823+568$ might be different from those derived in J05.

Figure 15 shows the dependence between bulk Lorentz factor and polarization variability index. There is a correlation between $\Gamma$ and $V_{\text {opt }}^{p}(r=0.53, \epsilon=0.1)$ and between $\Gamma$ and $V_{7 \mathrm{~mm}}^{p}$ $(r=0.72, \epsilon=0.05)$. Although $V_{3 \mathrm{~mm}}^{p}$ correlates with $\Gamma(r=0.42)$, this correlation is significant only at a level $\epsilon>0.15$. In the plane-wave shock model, the correlation is expected if the minimum degree of polarization corresponds to unshocked plasma and the maximum to faster, shocked plasma. In the shock-in-jet model, polarized regions at different wavelengths are partially cospatial and have essentially the same bulk Lorentz factor. Radiative energy losses of the relativistic electrons cause the shorter wavelength emission to occupy a smaller fraction of the full shocked region seen at longer wavelengths (Marscher \& Gear 1985; Marscher 1996). Since the shorter wavelength emission is assumed to originate from fewer turbulent cells, the degree of polarization and its variability should increase with decreasing wavelength according to equation (3).

Contrary to what we find at the other wavelengths, the polarization variability index at $1 \mathrm{~mm}$ does not correlate with the Lorentz factor ( $r=-0.12$; see Fig. 15). Furthermore, according to Table 12, the number of cells needed in the high-polarization state at $1 \mathrm{~mm}$ is only slightly larger than, or comparable to, that in the optical region. This conflicts with the expectations of the shock model, although $N_{\text {cell }}^{\max }$ at $1 \mathrm{~mm}$ is much smaller than at 3 and $7 \mathrm{~mm}$, in keeping with the model. The partial discrepancy suggests that another emission component in addition to transverse shocks is prominent at $1 \mathrm{~mm}$. A primary candidate is the "true" core, i.e., the bright, narrow end of the jet when observed at a wavelength where the emission is completely optically thin (Fig. 16). At wavelengths of $3 \mathrm{~mm}$ and longer, what appears to be the core is most likely a location at or outside the point where the optical depth $\tau(\nu) \sim 1$. The fact that the optical polarization does show the correlation, as well as the best alignment of position angle with the electric vector in the core at $7 \mathrm{~mm}$, implies that most of the nonthermal optical emission arises in shocks close to the $7 \mathrm{~mm}$ core rather than in the "true" core. This suggests that the "true" core does not possess relativistic electrons energetic enough to produce the optical synchrotron emission. 

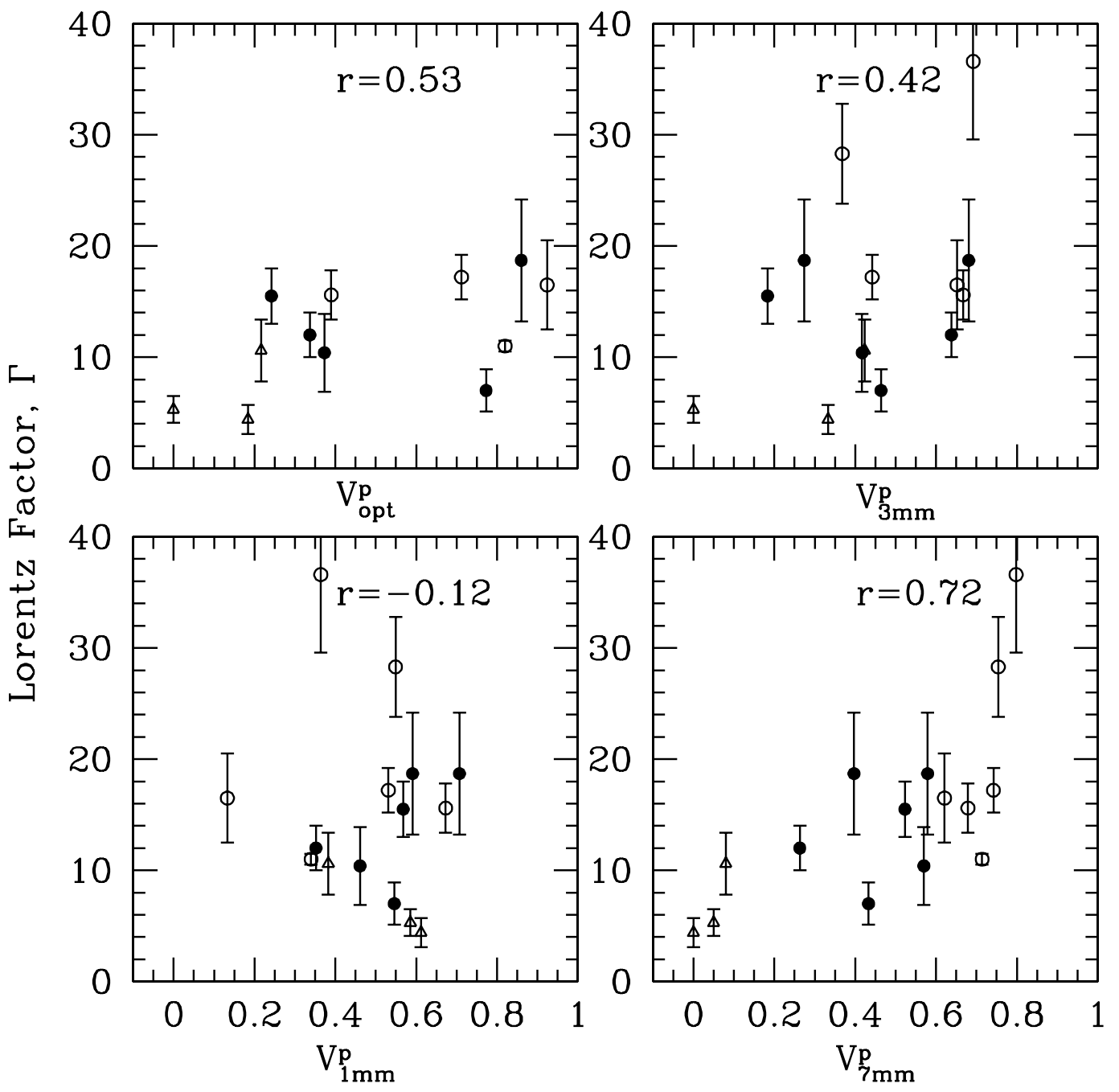

FIG. 15.-Lorentz factor vs. polarization variability index at different wavelengths. Triangles: LVP sources; filled circles: IVP sources; open circles: HVP sources.

\subsection{Magnetic Field Structure in the Jets}

In the shock-in-jet model (e.g., Marscher \& Gear 1985; Hughes et al. 1985), we expect a correlation between total flux and polarization owing to ordering of the magnetic field in the shocked region if the quiescent jet has a completely chaotic magnetic field. However, if the magnetic field in the unshocked region possesses a component parallel to the jet axis, a transverse shock will enhance the total flux but partially cancel the polarization as it compresses the turbulent component. In this case the polarized flux will at first decrease as the total flux rises. If the shocked emission grows strong enough that its polarized flux becomes greater than the initial polarized flux of the ambient jet, the EVPA will flip by $90^{\circ}$ after a minimum in polarized flux, and the polarized flux will subsequently grow with the total flux until the shocked emission weakens. The initial phase of decline in polarized flux may easily be missed in observations spaced such as ours. If an oblique shock is responsible for an outburst, its effect on the polarization will depend on the angle of the shock front relative to the ordered component of the ambient magnetic field, the viewing angle, and the bulk Lorentz factor of the jet. It is possible, in some cases, for the net polarization to increase, with the EVPA becoming closer to the position angle of the jet axis on the sky (Hughes 2005).
Figure 10 shows that the maximum of degree of polarization in the core at $7 \mathrm{~mm}$ tends to occur when the total flux rises, while at $1 \mathrm{~mm}$ the opposite relation is more probable. An increase of the total flux in the VLBI core is usually connected with the emergence of a new superluminal component from the optically thick part of the core (see J05). If these components represent transverse shock formation in the jet, they should display electric vectors aligned with the jet axis. On the other hand, $\chi_{1 \mathrm{~mm}}$ at minimum flux might reflect the magnetic field direction in the unshocked region. Figure 17 (left panel) displays the distributions of misalignment angles between the polarization and inner jet directions at times of minimum observed flux at $1 \mathrm{~mm}$. Figure 17 (middle panel) shows the distributions of offsets between $\chi_{7 \mathrm{~mm}}$ and the local jet direction for each superluminal component listed in Table 5 at the point when we can first separate the knot unambiguously from the core on the image (i.e., farther downstream than 0.1 mas). Figure 17 (right panel) presents the distribution of degree of polarization in the superluminal knots at their maximum total flux when the separation from the core $>0.1$ mas. Figure 17 indicates that (1) in the "quiescent jet" (prominent at minimum $1 \mathrm{~mm}$ flux), the magnetic field aligns with the jet for the LVP and HVP sources but is transverse to the jet in the IVP blazars; (2) in the shocked regions (superluminal knots) of the LVP objects, the magnetic field is longitudinal or oblique to the jet, 


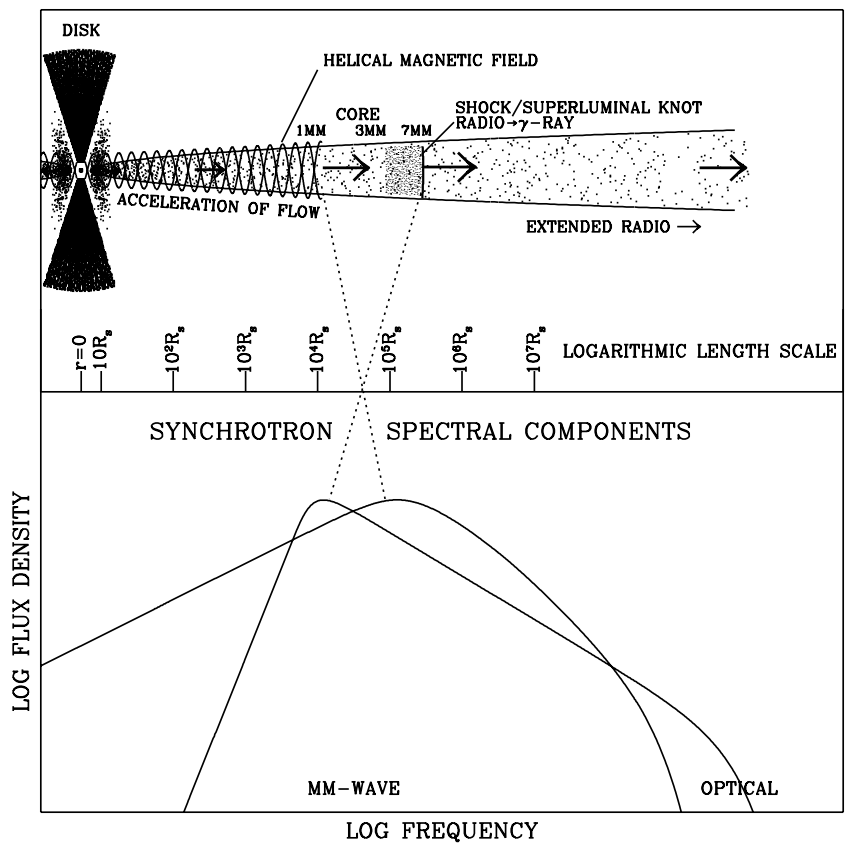

Fig. 16.- Sketch of the main emission regions in the compact jet compatible with the results of our investigation. Note the logarithmic scale of distance from the black hole (denoted by the dot at the center of the flared accretion disk). The magnetic field is a tight helix up to the "true" core at $1 \mathrm{~mm}$, beyond which it is chaotic. The system is symmetric with respect to the accretion disk (only one side is shown). The spectrum of the ambient jet rises gradually up to the peak frequency, at which the optical depth at the outer edge of the acceleration zone is of order unity, and cuts off at infrared frequencies. The spectrum of a shock moving through the core is more highly inverted below the peak, and its high-frequency cutoff extends beyond the optical region. Although only one is shown, multiple shocks are often present at any given time.

while for the IVP and HVP sources, its apparent direction is perpendicular to the jet.

The distributions of offsets between the electric vector and jet direction obtained for the IVP sources at different wavelengths (Fig. 13), the distribution of EVPAs in the superluminal knots, and the high polarization measured in the knots agree with the case of magnetic fields lying at oblique angles to the jet axis (Lister et al. 1998). (Relativistic aberration tends to align the polarization vectors closer to the jet axis, creating the illusion that the magnetic fields are closer to transverse than is actually the case in the flow frame of the emitting plasma.) This result matches the finding for BL Lac objects reported by Aller et al. (2003a, 2005), that both the linear polarization structure in VLBA images and the multifrequency polarized flux light curves obtained with singledish observations are consistent with the oblique shock hypothesis. Note that the IVP sources include most of the BL Lac objects plus those quasars with similar VLBI polarization structure.

In the HVP sources the distribution of offsets between the electric vector and jet direction at $1 \mathrm{~mm}$ is double peaked (Fig. 13), nearly parallel or perpendicular to the jet. According to Figure 17, at minimum flux at $1 \mathrm{~mm}$ the magnetic field has a parallel orientation, which could be associated with the unshocked plasma. A longitudinal component of the magnetic field in a quiescent jet can arise from a velocity shear across the jet flow (Laing 1980). Subtraction of the distribution shown in Figure 17 from that plotted in Figure 13 produces a distribution for the HVP sources at $1 \mathrm{~mm}$ that is quite similar to the distribution of the IVP sources. Therefore, the magnetic field in the regions responsible for enhanced emission, including superluminal knots, lies at a transverse or oblique angle to the jet axis, in agreement with the shock model. The superluminal components in the HVP sources (Fig. 17) have only a moderate degree of polarization $(<10 \%)$, indicating that even in the shocked region, the magnetic field is fairly turbulent. This is consistent with the high amplitude and short timescale of the polarization variability seen at all wavelengths. The existence of a longitudinal component of the magnetic field in quiescent states suggests that either an increase or a decrease in the degree of polarization is possible when a source brightens, depending on the shock strength responsible for the flux enhancement. This diversity of behavior reduces the possibility of observing a correlation between total flux and fractional polarization.

The general misalignment between the inner jet and the polarization vector in the core at $7 \mathrm{~mm}$ and at optical and $3 \mathrm{~mm}$ wavelengths for the HVP blazars (Fig. 13) fails to reveal any connection between the magnetic field and jet structure. In the shock model the distribution of electric vector offsets is expected to show either a good alignment with the jet direction (transverse or oblique shock; Hughes et al. 1985; Lister et al. 1998) or a bimodal shape (conical shock; Cawthorne \& Cobb 1990; Cawthorne 2006). In the conical shock wave model, the on-axis polarization in the shocked region corresponds to the upstream magnetic field structure, while the off-axis electric field vectors are oblique to the axis and cover a wide range of angles that depend on the shock cone opening angle and the viewing angle. A slight asymmetry of the jet cross-sectional structure with respect to the axis should lead to relaxation of the dependence of the magnetic field on the jet direction. Another factor that can contribute to the spread of $\chi$ with respect to the jet axis is that, despite the high resolution of the $7 \mathrm{~mm}$ images, the jet direction at the position where the emission occurs might be different from the direction inferred from the images. The latter implies that HVP sources possess higher bending of the jets on milliarcsecond scales than do IVP sources. Conical shocks might explain the appearance of stationary features in the jet (Gómez et al. 1997; Daly \& Marscher 1988), which are common in the HVP sources (J05). This model suggests that the core at $7 \mathrm{~mm}$ does not always represent the emission from the surface where the optical depth $\tau$ is of order unity. Rather, at any given frequency below the turnover in the spectrum, it would be either the $\tau \sim 1$ surface or the first standing conical shock system in the $\tau<1$ section of the jet, whichever is stronger. If so, the model would explain why the separation between the core and closest superluminal knot has a frequency dependence in some sources and not in others (see Marscher 2006 and references therein).

The distributions of position angle offsets between electric vectors and jet direction in the LVP sources (Figs. 13 and 17) indicate the existence of a persistent longitudinal component of the magnetic field in the emission regions at $3 \mathrm{~mm}, 7 \mathrm{~mm}$, in the quiescent jet, and in shocked regions. The modest degree of polarization observed in superluminal components of the LVP sources requires a very turbulent magnetic field even in the shocked region. J05 found that LVP sources have the widest jet opening angle in our sample, and the quasar 3C 273 shows significant variation in the apparent speed of superluminal components across the jet. The southern jet components are faster than the northern ones by a factor of 3 . This suggests that a longitudinal component of the magnetic field might arise from velocity shear in the ambient jet, or from complex evolution of the shock emission owing to the formation of oblique shocks combined with the effects of radiative transfer. The latter is seen in the numerical simulations of Hughes (2005).

Using the parameters of jet components $\mathrm{B} 1$ and $\mathrm{B} 2$ in the quasar $3 \mathrm{C} 273$ derived in J05 and the polarization information 


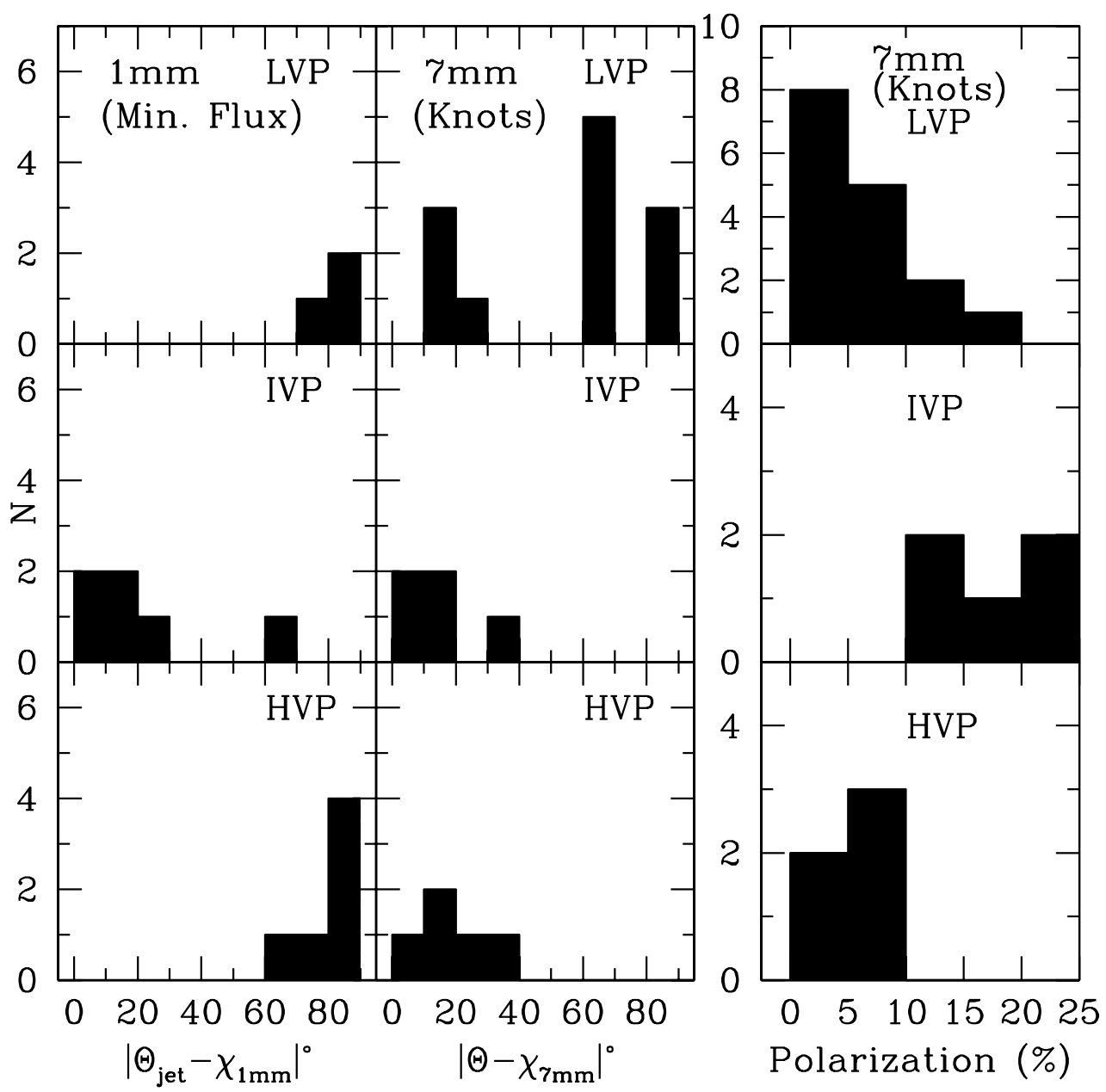

FIG. 17.-Left panels: Distributions of offsets between polarization position angle at $1 \mathrm{~mm}$ and inner jet at minimum flux observed at $1 \mathrm{~mm}$ in each source. Middle panels: Distributions of offsets between polarization position angle at $7 \mathrm{~mm}$ and local jet direction for superluminal knots listed in Table 5. Right panels: Distributions of the degree of polarization in superluminal knots (one entry for each knot; see text).

given in Table 5, we estimate the distance from the core, $\Delta z$, where a degree of polarization $p$ will be obtained as the result of velocity shear in the jet flow. We assume that the magnetic field starts out in a turbulent state with cell size $\Delta x: \Delta z / \Delta R \approx$ $\Gamma_{N} \Delta R p /\left(\Delta x \beta_{\text {rel }}\right)$, where $\Gamma_{N}$ is the bulk Lorentz factor at the northern side of the jet, $\Delta R$ is the half-width of the jet, and $\beta_{\text {rel }}$ is the relative speed between the northern and southern sides. In the plasma frame on the northern side (subscript $N), \beta_{\text {rel }}=$ $\left(\beta_{S}-\beta_{N}\right) /\left(1-\beta_{N} \beta_{S}\right)$, where $\beta=\left(1-\Gamma^{-2}\right)^{1 / 2}$. We assume that the Lorentz factor of knot B1 corresponds to the speed at the northern side $\left(\Gamma_{N}=8.3\right)$ and the Lorentz factor of knot B2 gives the speed on the southern side $\left(\Gamma_{S}=13.8\right)$. According to Table 5 , both components possess a similar maximum polarization $\left(p_{\mathrm{B} 1}=4.0 \%, p_{\mathrm{B} 2}=6.2 \%\right)$ when the direction of the electric vector is transverse to the jet axis, so we approximate $p \sim 5 \%$. Note that this polarization is achieved in both knots at the same distance from the core, $\sim 0.8$ mas. We use the half-opening and viewing angle of the jet derived in $\mathrm{J} 05\left(\theta=1.4^{\circ}\right.$ and $\Theta_{o}=6.1^{\circ}$, respectively). This exercise gives a ratio $\Delta R / \Delta x \sim 40$ and size of a turbulent cell $\Delta x \sim 0.001 \mathrm{pc}$. For the quasar 3C 273 we have obtained several groups of closely spaced observations at $3 \mathrm{~mm}$ near some VLBA epochs (see Table 3 ). The data reveal a very short timescale (computed according to the definition of Burbidge et al. 1974) of the fractional polarization variability at $3 \mathrm{~mm}$, $\Delta t_{\mathrm{var}} \sim 1.8$ days. This yields the size of the region responsible for the variability, $\Delta x_{\mathrm{var}} \sim 0.001 \mathrm{pc}$, which is consistent with the scale of turbulence in the magnetic field.
In summary, the sources in our sample show significant variety in the distributions of offsets between polarization position angle and jet direction. The observed behavior can be explained within the context of shock waves compressing highly turbulent magnetic fields and velocity shear stretching field lines along a direction parallel to the axis if the jet structure and kinematics on milliarcsecond scales are taken into account.

\subsection{Possibility of an Electromagnetically Dominated Core at $1 \mathrm{~mm}$}

The polarized emission at $1 \mathrm{~mm}$ possesses properties distinguished from the other wavelengths. (1) There is no correlation between fractional polarization variability index and Lorentz factor of the jet. (2) The maximum degree of polarization tends to occur when the total flux is lower than average. (3) The standard deviation of the mean of the minimum degrees of polarization over sources in a variability group is lower than expected in the cellular model of magnetic field structure. An inverse relationship between the degree of polarization and total flux is expected in electromagnetically dominated (ED) jets (Lovelace \& Romanova 2003 ) if the plasma responsible for a rise in the total flux has a more chaotic magnetic field than that of the ED section. This can be the case even if a shock orders an underlying turbulent field in the same sense as does the mostly toroidal field in the ED portion of the jet, since the polarization of the ED region should be quite high.

The upstream ED model of the jet is a promising scenario since it explains the high Lorentz factors of the flow needed to 
produce the high apparent speeds seen in some blazars. Acceleration of the jet to such high values of $\Gamma$ is accomplished by conversion of Poynting flux to flow energy through the Lorentz force that occurs on scales that are much larger than the gravitational radius of the central black hole (e.g., Vlahakis 2006). The tentative anticorrelation between total flux and degree of polarization at $1 \mathrm{~mm}$ and rather positive correlation between the values at $3 \mathrm{~mm}$ and in the core at $7 \mathrm{~mm}$ suggest that the acceleration might end between the VLBI cores at 1 and $3 \mathrm{~mm}$. In this case the $1 \mathrm{~mm}$ emission region should possess a large-scale, mostly toroidal, magnetic field component, which in the plane of the sky should lie either transverse or longitudinal to the jet direction (Lyutikov et al. 2005). This is consistent with the distributions of EVPAs at $1 \mathrm{~mm}$ with respect to the jet direction in the IVP and HVP sources, in which emission from the VLBI core at $1 \mathrm{~mm}$ seems to dominate the overall source emission at $1 \mathrm{~mm}$ (Fig. 13). If the section of the jet seen at 3 and $7 \mathrm{~mm}$ is beyond the transition point where the jet becomes mainly hydrodynamical and turbulent, then the polarization behavior at these wavelengths will be similar to that discussed in $\S \S 8.1-8.3$, with shocks partially ordering the randomly oriented component of the magnetic field. The behavior of the optical polarization is more similar to that at 3 and $7 \mathrm{~mm}$, and the best agreement between polarization position angles for simultaneous observations is observed between the optical EVPA and polarization position angle in the core at $7 \mathrm{~mm}$. This suggests that the optical emission originates in shocks at or downstream of the transition point, e.g., at or between the VLBI cores at 3 and $7 \mathrm{~mm}$, and implies that high-energy particle acceleration is more efficient in shocks than in the ED region.

\section{SUMMARY}

We have performed multifrequency, multiepoch, quasisimultaneous linear polarization observations of a sample of AGNs that possess highly relativistic jets and high levels of polarization at $1 \mathrm{~mm}$. We separate the sample into three groups according to the pattern of variability of the polarization in the VLBI core at $7 \mathrm{~mm}$. This classification appears to be connected with physical differences between the objects. However, the sample is small and future observations of a larger sample are needed to confirm the findings. The properties of the groups are as follows:

1. The objects with low variability of polarization (LVP) in the VLBI core unite two radio galaxies with superluminal speeds on parsec scales and a quasar with low observed optical polarization. The LVP sources have measured optical polarization $<2 \%$ and radio core polarization $<1 \%$ and produce strongly polarized radio jet components that are responsible for the high polarization observed at $1 \mathrm{~mm}$. The LVP inner jets are most likely subjected to very high RMs, RM $>5 \times 10^{5} \mathrm{rad} \mathrm{m}^{-2}$, that cause depolarization of the core region at millimeter wavelengths. The optical nonthermal emission is greatly diluted by nonsynchrotron components, probably associated with the big blue bump. The parsec-scale jets are characterized by moderate Lorentz factors, near the low end of the blazar range, and by viewing and opening angles that are larger than those of a typical blazar. The distributions of EVPAs at $3 \mathrm{~mm}$ and in the inner jet at $7 \mathrm{~mm}$ with respect to the jet axis reveal a preferred longitudinal component of the magnetic field that can be explained by velocity shear.

2. The sources with intermediate variability of polarization (IVP) in the VLBI core include BL Lac objects and quasars with preferred direction of the electric vector at all wavelengths close to the direction of the inner jet. They possess very high optical polarization ( $\gtrsim 30 \%$ ) and relatively highly polarized ( $>2 \%$ ) VLBI cores at $7 \mathrm{~mm}$. The polarized and total flux density spectra from optical wavelengths to $1 \mathrm{~mm}$ suggest that synchrotron components with fairly flat spectra, $\alpha \sim 0.5$, dominate the total emission at these wavelengths. The IVP sources possess a moderate $\mathrm{RM}, \mathrm{RM} \lesssim 5 \times 10^{3} \mathrm{rad} \mathrm{m}^{-2}$, in the core, which is consistent with the lack of emission lines in the spectra of BL Lac objects owing to a relatively low column density of gas. The radio jets of the IVP sources are highly relativistic, with $\Gamma \geq 10$. The alignment of the polarization with the jet at all wavelengths indicates the dominance of a transverse or (in the plasma frame) oblique component of the magnetic field.

3. The category of objects with highly variable polarization (HVP) in the VLBI core consists of the OVV quasars and the BL Lac object OJ 287. These display strong variations in both degree and polarization position angle. The very high optical polarization seen in the IVP sources occurs in the HVP sources, but, in contrast with the IVP blazars, low polarization $(<1 \%)$ at millimeter wavelengths and in the VLBI core is common. Only a single synchrotron component with a steep spectral index, $\alpha \sim 1.0$, is required to produce the polarized emission from optical to $1 \mathrm{~mm}$ wavelengths, although a nonsynchrotron component could contribute a small fraction of the total optical emission. The HVP sources possess high RMs in the VLBI core at $7 \mathrm{~mm}$, $\mathrm{RM} \sim 3 \times 10^{4} \mathrm{rad} \mathrm{m}^{-2}$, implying that the core is surrounded by dense thermal plasma. The radio jets of the HVP sources have Lorentz factors at the high-end tail of the blazar distribution. The general misalignment between the polarization position angle and jet direction indicates that there is little, if any, connection between the two.

The diversity of polarization properties of the sources in the sample allows us to establish connections between parameters at different wavelengths. (1) There is good agreement between the optical EVPA and that in the VLBI core at $7 \mathrm{~mm}$ for the IVP and HVP blazars. (2) The overall polarization of a source at 1 and $3 \mathrm{~mm}$ strongly correlates with the degree of polarization in the inner jet of the LVP sources. (3) The EVPA at 1 and $3 \mathrm{~mm}$ corresponds to that in the inner jet for the LVP sources. (4) There is a strong correlation between the level of polarization variability in the optical region and in the VLBI core at $7 \mathrm{~mm}$. (5) The optical and $7 \mathrm{~mm}$ polarization variability indices correlate with the bulk Lorentz factor of the radio jets. (6) The degree of polarization decreases with wavelength. (7) The distributions of electric vector offsets with respect to the inner jet direction at optical and millimeter wavelengths are similar within each variability group, with distinct differences among the three groups.

In general, the time variability across the various wavelengths follows the expectations of models in which rather weak shock waves propagate down a jet containing a turbulent magnetic field. After taking into account relativistic aberration, we find that the shock fronts generally lie at oblique angles to the jet axis.

The correlations that we have uncovered tightly link the emission at optical and $3 \mathrm{~mm}$ wavelengths to the VLBI core at $7 \mathrm{~mm}$. At $1 \mathrm{~mm}$ there is a possible anticorrelation between total flux density and degree of polarization, but not at 7 or $3 \mathrm{~mm}$. This suggests the presence of a well-ordered magnetic field in a region that is optically thick at $\lambda \gtrsim 1 \mathrm{~mm}$ but does not contain electrons with energies sufficiently high to radiate at optical wavelengths. During outbursts, the $1 \mathrm{~mm}$ polarization is weakened by the somewhat more weakly polarized emission from a shock moving through the downstream portion of the jet where the ambient magnetic field is chaotic. We suggest that the quasisteady $1 \mathrm{~mm}$ emission arises near the end of the section where the jet is accelerated by magnetic forces, a process that requires a tight helical 
field geometry. The acceleration of electrons in this region is apparently inadequate to produce optical synchrotron radiation; shocks might be required to do this. Figure 16 presents a sketch of a relativistic jet to illustrate our interpretation.

Our study demonstrates the utility of multi-wave-band polarization monitoring of blazars when combined with multiepoch imaging of their jets with VLBI at millimeter wavelengths. Further progress is possible through more intense time and frequency coverage of a large number of objects.

This material is based on work supported by the National Science Foundation under grant AST 04-06865. P. S. S. acknowledges support from National Aeronautics and Space Adminis- tration contract 1256424 . T. V. C. and A. M. S. acknowledge support through research grants from the UK Particle Physics and Astronomy Research Council. J. L. G. acknowledges support from the Spanish Ministerio de Educación y Ciencia and the European Fund for Regional Development through grant AYA2004-08067-C03-03. We thank Gary Schmidt for the maintenance and use of the Two-Holer Polarimeter. The VLBA is a facility of the National Radio Astronomy Observatory, operated by Associated Universities, Inc., under cooperative agreement with the National Science Foundation. The James Clerk Maxwell Telescope is operated by The Joint Astronomy Centre on behalf of the Particle Physics and Astronomy Research Council of the United Kingdom, the Netherlands Organisation for Scientific Research, and the National Research Council Canada.
Aller, H. D., Aller, M. F., \& Hughes, P. A. 2003a, in ASP Conf. Ser. 300, Astronomy at the Fringe, ed. J. A. Zensus, M. H. Cohen, \& E. Ross (San Francisco: ASP), 143

Aller, H. D., Aller, M. F., Hughes, P. A., Homan, D. C., Wardle, J. F. C., \& Roberts, H. D. 2005, in ASP Conf. Ser. 340, Future Directions in High Resolution Astronomy: The 10th Anniversary of the VLBA, ed. J. D. Romney \& M. J. Read (San Francisco: ASP), 165

Aller, M. F., Aller, H. D., \& Hughes, P. A. 2003b, ApJ, 586, 33

Attridge, J. M., Wardle, J. F. C., \& Homan, D. C. 2005, ApJ, 633, L85

Begelman, M. C., Blandford, R. D., \& Rees, M. J. 1984, Rev. Mod. Phys., 56, 255

Bowker, A. H., \& Lieberman, G. J. 1972, Engineering Statistics (Englewood Cliffs: Prentice-Hall)

Burbidge, G. R., Jones, T. W., \& Odell, S. L. 1974, ApJ, 193, 43

Burch, S. F. 1979, MNRAS, 186, 519

Burn, B. J. 1966, MNRAS, 133, 67

Cawthorne, T. V. 2006, MNRAS, 367, 851

Cawthorne, T. V., \& Cobb, W. K. 1990, ApJ, 350, 536

Cawthorne, T. V., \& Wardle, J. F. C. 1988, ApJ, 332, 696

Cawthorne, T. V., Wardle, J. F. C., Roberts, D. H., \& Gabuzda, D. C. 1993, ApJ, 416, 519

Daly, R. A., \& Marscher, A. P. 1988, ApJ, 334, 539

D'Arcangelo, F. D., et al. 2007, ApJ, 659, L107

Gabuzda, D. C. 2006, in ASP Conf. Ser. 345, From Clark Lake to the Long Wavelength Array: Bill Erickson's Radio Science, ed. N. Kassim et al. (San Francisco: ASP), 264

Gabuzda, D. C., Rastorgueva, E. A., Smith, P. S., \& O'Sullivan, S. P. 2006, MNRAS, 369, 1596

Gabuzda, D. C., Sitko, M. L., \& Smith, P. S. 1996, AJ, 112, 1877

Gómez, J. L., Marscher, A. P., Alberdi, A., Jorstad, S. G., \& García-Miró, C. 2000, Science, 289, 2317

Gómez, J. L., Martí, J. M., Marscher, A. P., Ibáñez, J. M., \& Alberdi, A. 1997, ApJ, 482, L33

Gracia, J., Tsinganos, K., \& Bogovalov, S. V. 2005, A\&A, 442, L7

Greaves, J. S., et al. 2003, MNRAS, 340, 353

Hagen-Thorn, V. A. 1980, Ap\&SS, 73, 279

Holland, W. S., et al. 1999, MNRAS, 303, 659

Hughes, P. A. 2005, ApJ, 621, 635

Hughes, P. A., Aller, H. D., \& Aller, M. F. 1985, ApJ, 298, 301

- 1989, ApJ, 341, 68

Hughes, P. A., \& Miller, L. 1991, in Beams and Jets in Astrophysics, ed. P. A. Hughes (Cambridge: Cambridge Univ. Press), 1

Impey, C. D., Malkan, \& M. A., \& Tapia, S. 1989, ApJ, 347, 96

Impey, C. D., \& Neugebauer, G. 1988, AJ, 95, 307

Jorstad, S. G., Marscher, A. P., Mattox, J. R., Wehrle, A. E., Bloom, S. D., \& Yurchenko, A. V. 2001, ApJS, 134, 181

Jorstad, S. G., et al. 2005, AJ, 130, 1418 (J05)

Kellermann, K. I., et al. 2004, ApJ, 609, 539

Laing, R. A. 1980, MNRAS, 193, 439

Landolt, A. U. 1983, AJ, 88, 439

Lister, M. L. 2006, in ASP Conf. Ser. 350, Blazar Variability Workshop II: Entering the GLAST Era, ed. H. R. Miller et al. (San Francisco: ASP), 139
Lister, M. L., \& Homan, D. C. 2005, AJ, 130, 1389

Lister, M. L., Marscher, A. P., \& Gear, W. K. 1998, ApJ, 504, 702

Lister, M. L., \& Smith, P. S. 2000, ApJ, 541, 66

Lobanov, A. P. 1998, A\&A, 330, 79

Lovelace, R. V. E., \& Romanova, M. M. 2003, ApJ, 596, L159

Lyutikov, M., Pariev, V. I., \& Gabuzda, D. C. 2005, MNRAS, 360, 869

Marscher, A. P. 1996, in ASP Conf. Ser. 110, Blazar Continuum Variability, ed. H. R. Miller, J. R. Webb, \& J. C. Noble (San Francisco: ASP), 248 2006, in AIP Conf. Proc. 856, Relativistic Jets: The Common Physics of AGN, Microquasars, and Gamma Ray Bursts, ed. P. A. Hughes \& J. N. Bregman (New York: AIP), 1

Marscher, A. P., \& Gear, W. K. 1985, ApJ, 298, 114

McKinney, J. C. 2006, MNRAS, 367, 1797

Mead, A. R. G., Ballard, K. R., Brand, P. W. J. L., Hough, J. H., Brindle, C., \& Bailey, J. A. 1990, A\&AS, 83, 183

Meier, D. L., Koide, S., \& Uchida, Y. 2001, Science, 291, 84

Meier, D. L., \& Nakamura, N. 2006, in ASP Conf. Ser. 350, Blazar Variability Workshop II: Entering the GLAST Era, ed. H. R. Miller et al. (San Francisco: ASP), 195

Moore, M. L., Schmidt, G. D., \& West, S. C. 1987, ApJ, 314, 176

Nartallo, R., Gear, W. K., Murray, A. G., Robson, E. I., \& Hough, J. H. 1998, MNRAS, 297, 667

Pacholczyk, A. G. 1970, Radio Astrophysics (San Francisco: W. H. Freeman)

Savolainen, T., Wiik, K., Valtaoja, E., Jorstad, S. G., \& Marscher, A. P. 2002, A\&A, 394, 851

Sitko, M. L., Schmidt, G. D., \& Stein, W. A. 1985, ApJS, 59, 323

Smith, P. S. 1996, in ASP Conf. Ser. 110, Blazar Continuum Variability, ed. H. R. Miller, J. R. Webb, \& J. C. Noble (San Francisco: ASP), 135

Smith, P. S., \& Balonek, T. J. 1998, PASP, 110, 1164

Smith, P. S., Balonek, T. J., Elston, R., \& Heckert, P. A. 1987, ApJS, 64, 459

Smith, P. S., Balonek, T. J., Heckert, P. A., Elston, R., \& Schmidt, G. D. 1985, AJ, 90, 1184

Smith, P. S., Elston, R., Berriman, G., Allen, R. G., \& Balonek, T. J. 1988, ApJ, 326, L39

Smith, P. S., Hall, P. B., Allen, R. G., \& Sitko, M. L. 1992, ApJ, 400, 115

Smith, P. S., Schmidt, G. D., \& Allen, R. G. 1993, ApJ, 409, 604

Taylor, G. B. 1998, ApJ, 506, 637 . 2000, ApJ, 533, 95

Vlahakis, N. 2006, in ASP Conf. Ser. 350, Blazar Variability Workshop II: Entering the GLAST Era, ed. H. R. Miller et al. (San Francisco: ASP), 169 Vlahakis, N., \& Königl, A. 2004, ApJ, 605, 656

Wardle, J. F. C., Cawthorne, T. V., Roberts, D. H., \& Brown, L. F. 1994, ApJ, 437,122

Wardle, J. F. C., \& Kronberg, P. P. 1974, ApJ, 194, 249

Wills, B. J., Wills, D., Breger, M., Antonucci, R. R. J., \& Barvainis, R. 1992, ApJ, 398, 454

ApJ, 2003, ApJ, 589, 126

Zavala, R. T., \& Taylor, G. B. 2003, ApJ, 589, 126

2004, ApJ, 612, 749

2005, ApJ, 626, L73 